Florida International University FIU Digital Commons

$11-12-2009$

\title{
Energy-Efficient Self-Organization of Wireless Acoustic Sensor Networks for Ground Target Tracking
}

Malaka J. Walpola

Florida International University, malakajw@yahoo.com

DOI: 10.25148/etd.FI09120830

Follow this and additional works at: https://digitalcommons.fiu.edu/etd

\section{Recommended Citation}

Walpola, Malaka J., "Energy-Efficient Self-Organization of Wireless Acoustic Sensor Networks for Ground Target Tracking" (2009). FIU Electronic Theses and Dissertations. 129.

https://digitalcommons.fiu.edu/etd/129 


\section{FLORIDA INTERNATIONAL UNIVERSITY}

Miami, Florida

\section{ENERGY-EFFICIENT SELF-ORGANIZATION OF WIRELESS ACOUSTIC SENSOR NETWORKS FOR GROUND TARGET TRACKING}

A dissertation submitted in partial fulfillment of the requirements for the degree of DOCTOR OF PHILOSOPHY in ELECTRICAL ENGINEERING by

Malaka Jayathu Walpola 


\section{To: Dean Amir Mirmiran}

College of Engineering and Computing

This dissertation, written by Malaka Jayathu Walpola, and entitled Energy-Efficient Self-Organization of Wireless Acoustic Sensor Networks for Ground Target Tracking, having been approved in respect to style and intellectual content, is referred to you for judgment.

We have read this dissertation and recommend that it be approved.

Chenzhong Li

Hao Zhu

Jinsong Zhang, Co-Major Professor

Kang K. Yen, Co-Major Professor

Date of Defense: November 12, 2009

The dissertation of Malaka Jayathu Walpola is approved.

Dean Amir Mirmiran
College of Engineering and Computing

Florida International University, 2009 


\section{DEDICATION}

To my loving family, for their encouragements, sacrifices and unconditional love and support that helped me reach this important milestone in my life. 


\section{ACKNOWLEDGMENTS}

Motivation and commitment are the greatest assets of a human's life to reach the milestone of success. Love, support and encouragement are the jewels that make the journey to success possible and memorable. I take this opportunity to convey my sincere thanks and gratitude to my advisors, committee members, teachers, family and friends for their guidance, support, encouragement and love through the entire process. Without them the successful completion of my doctoral studies would not have been possible.

I convey my deepest gratitude and appreciation to my co-major advisor Dr. Jinsong Zhang for his valuable advising and guidance from the beginning of the pursuit of my $\mathrm{PhD}$ degree. He introduced me to the field of wireless sensor networks and was influential in guiding me throughout my $\mathrm{PhD}$ research, which was pivotal to my research achievements. I am thankful for his patience, understanding, motivation and friendship during the challenging times of my doctoral studies.

I express my sincere thanks and gratitude to my co-major advisor Professor Kang K. Yen for his guidance and precious comments that helped me overcome obstacles during my research. His guidance and direction was invaluable for my research accomplishments. I am especially thankful for his willingness and dedication in providing me with valuable feedback of my research work.

I am indebted to Dr. Hao Zhu for his encouragement and valuable advice during my doctoral studies. The discussions that I had with him provided invaluable knowledge, which was a strong helping hand to successfully overcome difficulties in my research. I wish to thank him for the directions and encouragements he provided me during my doctoral studies. 
I am greatly thankful to dissertation committee member, Dr. Chenzhong Li for his precious comments and time to assist me in my $\mathrm{PhD}$ research. I take this opportunity to convey my deepest appreciation to Dr. Chi Zhou for her guidance during the initial period of my doctoral studies.

I wish to express my sincere gratitude to Dr. David Roelant for mentoring and guiding me during my $\mathrm{PhD}$ research and research assistantship at Applied Research Center. His invaluable advices were influential in enhancing my knowledge and skills. I am obliged to Dr. Jinsong Zhang, Dr. David Roelant and the Applied Research Center, for providing me financial support throughout my doctoral studies.

My sincere appreciation goes to Ms. Maria Benincasa, for her unyielding commitment to help me in administrative tasks throughout my PhD studies. I am grateful to Ms. Pat Brammer, Ms. Amy Diaz, Ms. Kim Cole, Ms. Gloria Dingeldein, Ms. Claudia Benitez, and Ms. Patty Cepero for their administrative assistance. I would like to thank Dr. George Philippidis, Mr. Tomas Pribanic, Dr. Dwayne McDaniel, Dr. Leonel Lagos, Mr. Jose Varona and Mr. Ravi Guddavali for their support during my stay at the Applied Research Center.

I extend my thanks to all developers and contributors of the ns-2 distributed event simulator for providing and maintaining a high quality simulation environment, which has been a great help for my research work. My special thanks go to Dr. Brad Karp for making available the implementation of the GPSR on ns-2 simulator, which was helpful for my simulations.

Last but not least, I convey my deepest gratitude and appreciation to my family and friends for their continuous patience, love and support. I am especially thankful to 
Ms. Vishaka Nanayakkara, Dr. Rukshan Athauda, and Mr. Kasun Wickramaratna for their support and advice in pursuing my doctoral studies. I am grateful to my collogues, Dr. Zeiwan Wan, Dr. Te-Shun Chou, Mr. Lakshitha Weerakkody, Mr. Kishnathej Vedala, Mr. Serkan Akar and Ms. Liwei An for the help and friendship during my studies at Florida International University. A special thank is due for Dr. Zeiwan Wan for providing the implementation of her communication stack on ns- 2 and supporting me during my research tasks. My sincere gratitude and thanks goes to my teachers who have been the guiding light of my life. I express my heart-felt gratitude and love to my dear parents and brother for standing by me during my entire life and encouraging and motivating me during difficult times. I am grateful to my aunts, uncles and grandparents for the strong foundation they established in my education and life. I am especially thankful to my loving wife for her support, love and affection that strengthened me to face the challenges during my journey of life. Finally, I am thankful to everyone for their love and support that has helped me to successfully reach this milestone in my life. 


\title{
ABSTRACT OF THE DISSERTATION \\ ENERGY-EFFICIENT SELF-ORGANIZATION OF WIRELESS ACOUSTIC SENSOR NETWORKS FOR GROUND TARGET TRACKING
}

\author{
by \\ Malaka Jayathu Walpola \\ Florida International University, 2009 \\ Miami, Florida \\ Professor Jinsong Zhang, Co-Major Professor \\ Professor Kang K. Yen, Co-Major Professor
}

With the developments in computing and communication technologies, wireless sensor networks have become popular in wide range of application areas such as health, military, environment and habitant monitoring. Moreover, wireless acoustic sensor networks have been widely used for target tracking applications due to their passive nature, reliability and low cost. Traditionally, acoustic sensor arrays built in linear, circular or other regular shapes are used for tracking acoustic sources. The maintaining of relative geometry of the acoustic sensors in the array is vital for accurate target tracking, which greatly reduces the flexibility of the sensor network. To overcome this limitation, we propose using only a single acoustic sensor at each sensor node. This design greatly improves the flexibility of the sensor network and makes it possible to deploy the sensor network in remote or hostile regions through air-drop or other stealth approaches. Acoustic arrays are capable of performing the target localization or generating the bearing estimations on their own. However, with only a single acoustic sensor, the sensor 
nodes will not be able to generate such measurements. Thus, self-organization of sensor nodes into virtual arrays to perform the target localization is essential.

We developed an energy-efficient and distributed self-organization algorithm for target tracking using wireless acoustic sensor networks. The major error sources of the localization process were studied, and an energy-aware node selection criterion was developed to minimize the target localization errors. Using this node selection criterion, the self-organization algorithm selects a near-optimal localization sensor group to minimize the target tracking errors. In addition, a message passing protocol was developed to implement the self-organization algorithm in a distributed manner. In order to achieve extended sensor network lifetime, energy conservation was incorporated into the self-organization algorithm by incorporating a sleep-wakeup management mechanism with a novel cross layer adaptive wakeup probability adjustment scheme. The simulation results confirm that the developed self-organization algorithm provides satisfactory target tracking performance. Moreover, the energy saving analysis confirms the effectiveness of the cross layer power management scheme in achieving extended sensor network lifetime without degrading the target tracking performance. 


\section{TABLE OF CONTENTS}

CHAPTER PAGE

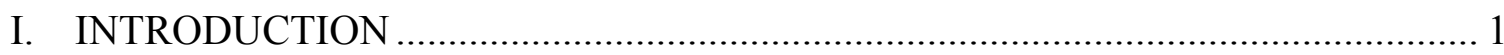

1.1 Background and Motivation...................................................................... 1

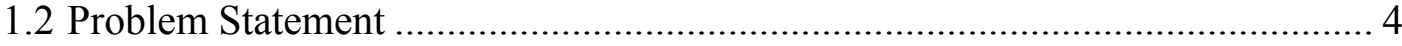

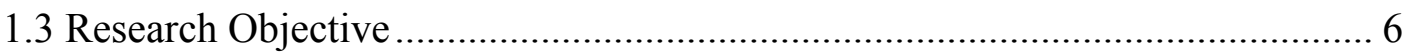

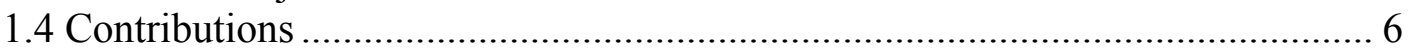

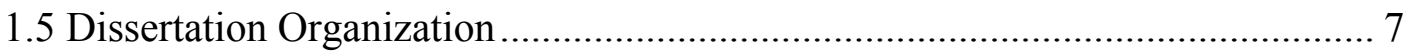

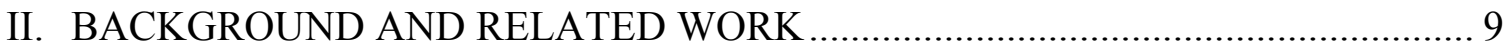

2.1 Acoustic Source Localization Strategies …………........................................ 9

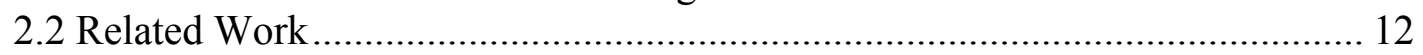

2.2.1 Target Tracking Using Wireless Sensor Networks ................................ 12

2.2.2 Energy Efficient Target Tracking Using Wireless Sensor

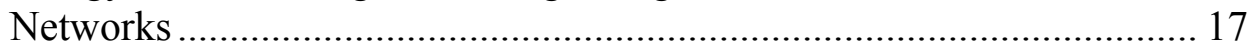

2.2.3 Energy Efficiency in Wireless Sensor Networks .................................... 28

2.2.3.1 Energy Efficiency in Network Layer ............................................. 29

2.2.3.2 Energy Efficiency in MAC Layer .................................................... 35

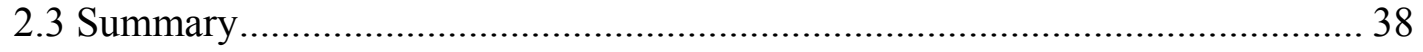

III. TARGET LOCALIZATION AND TRACKING.................................................. 40

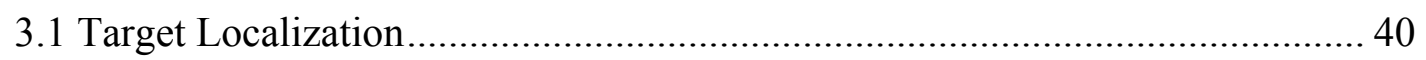

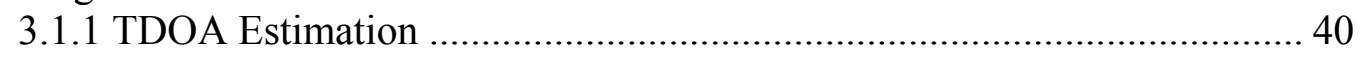

3.1.2 Target Localization............................................................................. 41

3.2 Localization Error Source Analysis............................................................... 44

3.2.1 TDOA Estimation Error ....................................................................... 45

3.2.2 Bearing Estimation Error …………………………........................... 47

3.2.3 Triangulation Error.......................................................................... 47

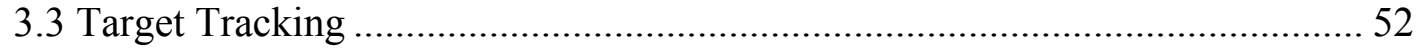

IV. THE ENERGY-EFFICIENT AND DISTRIBUTED

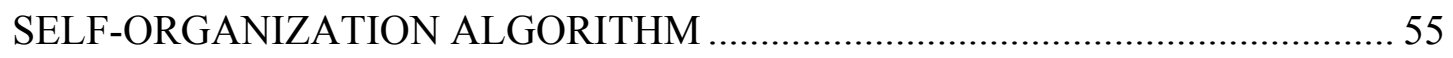

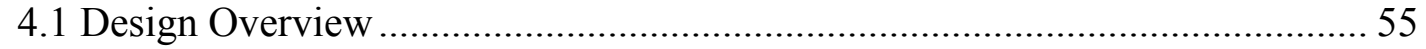

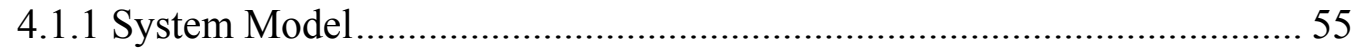

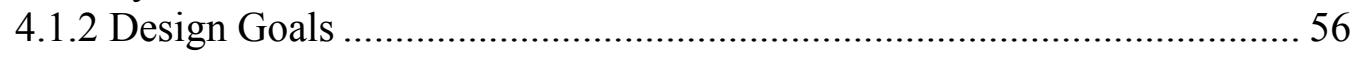

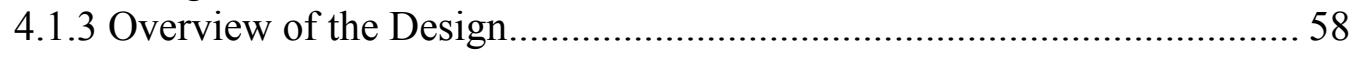

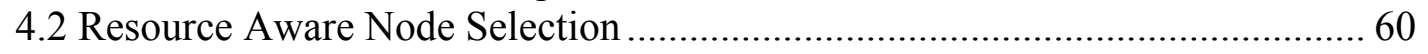

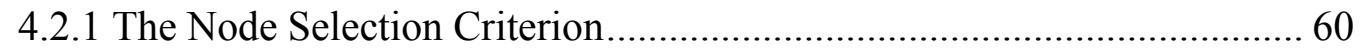

4.2.2 Node Selection with Global Information .................................................. 62

4.2.3 Resource Aware Node Selection Process.................................................... 63

4.2.4 Incorporation of Energy Balancing into the Node Selection ..................... 67

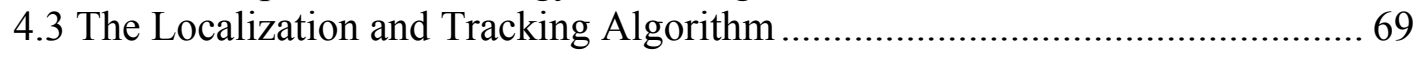

4.3.1 Initialization of the Localization Sensor Group ………............................. 69 


\subsubsection{Initial Four Closest Nodes Localization Sensor}

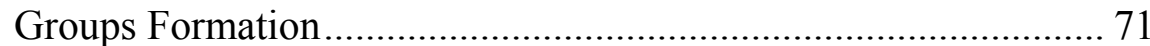

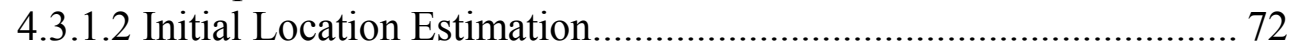

4.3.1.3 Localization Sensor Group Formation .............................................. 72

4.3.2 Target Localization and Tracking ........................................................ 74

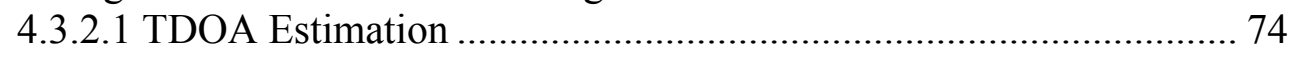

4.3.2.2 Target Localization ................................................................... 74

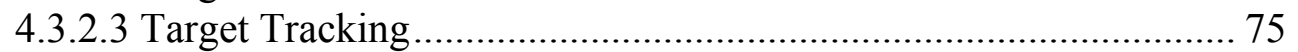

4.3.2.4 Localization Sensor Group Evaluation ........................................ 75

4.3.3 Localization Sensor Group Update ......................................................... 77

4.3.3.1 Desirable Geometry Formation of Master Sensor

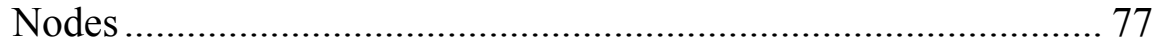

4.3.3.2 Search for the Two Master Nodes................................................... 80

4.3.3.3 Localized Searches for the Two Slave Nodes................................... 81

4.4 Message Passing for Energy-Efficient and Distributed

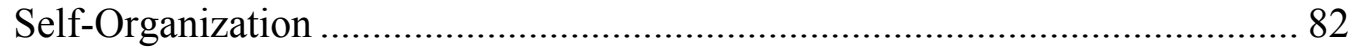

4.4.1 Initialization of the Localization Sensor Group ……………………….... 82

4.4.1.1 Initial Four Closest Nodes Localization Sensor

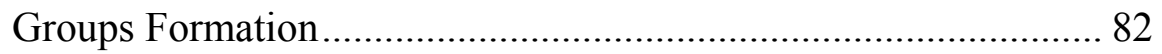

4.4.1.2 Initial Groups Discovery .................................................................. 85

4.4.1.3 TDOA Information Exchange for Initial Location

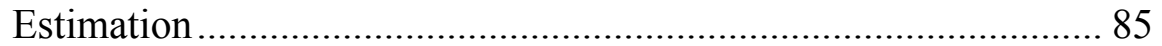

4.4.1.4 Search for Master Nodes.................................................................. 86

4.4.1.5 Localized Search for Individual Slave Nodes ................................. 88

4.4.2 Target Localization and Tracking ....................................................... 89

4.4.3 Localization Sensor Group Update …………….................................... 90

4.5 Energy Conservation ........................................................................................ 92

4.5.1 Energy Conservation Using Sleep-wakeup Management

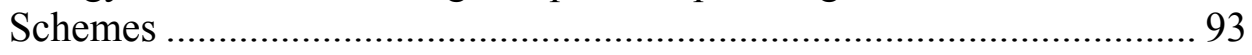

4.5.2 Energy Efficient and Latency Sensitive MAC Protocol ........................... 94

4.5.2.1 The Opportunistic Sleep Scheme.................................................. 95

4.5.2.2 The Anycast Based Data Forwarding Scheme................................. 96

4.5.3 Cross Layer Power Management ............................................................ 98

4.5.3.1 Rationale for Cross Layer Power Management ............................... 98

4.5.3.2 Cross Layer Wakeup Probability Adjustment Scheme.................... 99

4.6 Cross Layer Enhancements .......................................................................... 104

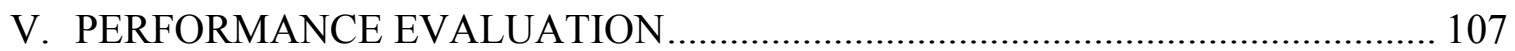

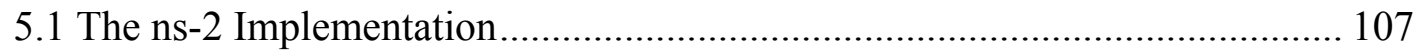

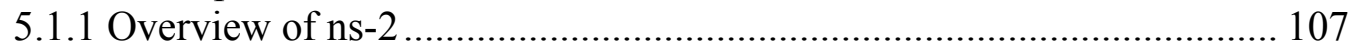

5.1.2 Implementation of Self-Organization Algorithm in ns-2 ....................... 109

5.1.2.1 Implementation of the DSOA Application Agent.......................... 111

5.1.2.2 Modification of GPSR to Implement Interface for

the Application Agent............................................................... 112

5.1.2.3 Modifications to the ns-2 Energy Model ...................................... 112 
5.1.3 Incorporating Energy Conservation ....................................................... 113

5.1.3.1 Wakeup Probability Adjustment Modification ............................... 114

5.1.3.2 Implementation of the Cross Layer Enhancements ...................... 115

5.1.4 Implementation of Tracking Using Four Closest Nodes

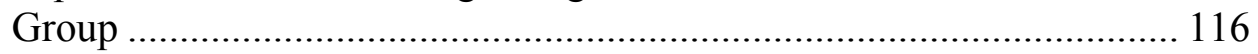

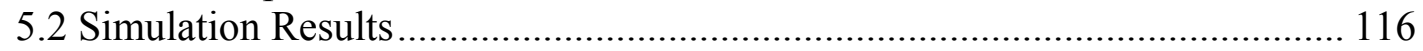

5.2.1 Target Tracking Performance.............................................................. 117

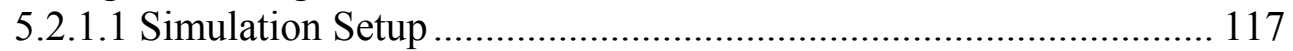

5.2.1.2 Results and Discussion.................................................................. 121

5.2.2 Effect of Application Level Energy Consideration................................. 124

5.2.2.1 Simulation Setup .................................................................... 124

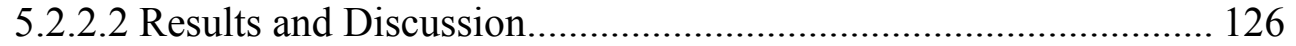

5.2.3 Evaluation of the Developed Energy Conservation Scheme................... 131

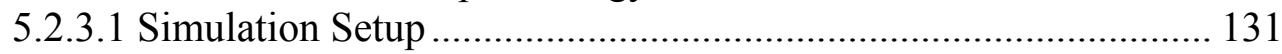

5.2.3.2 Results and Discussion................................................................. 133

5.2.4 The Selection of Localization Sensor Group …………........................ 142

5.2.5 Effect of Sensor Node Density ............................................................ 146

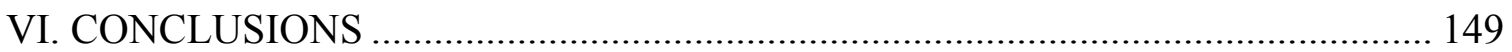

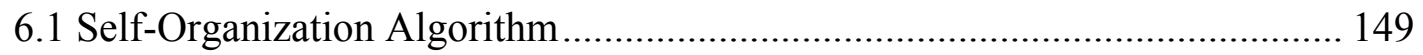

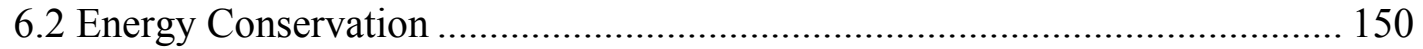

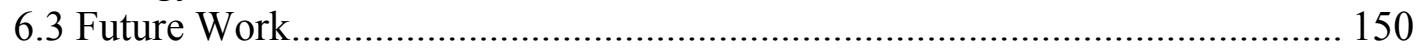

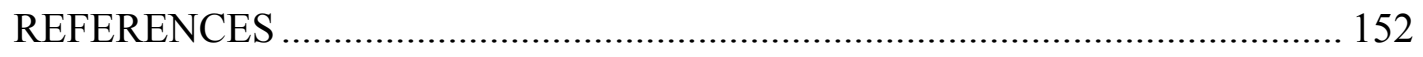

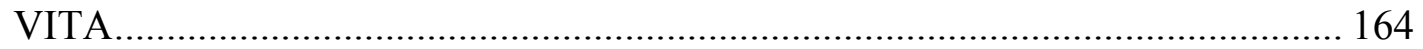




\section{LIST OF TABLES}

TABLE

PAGE

Table 3.1 Position Estimation Error Statistics for Different Bearing Angles 50

Table 3.2 Position Estimation Error Statistics for Different Distances from Sensor Pair Midpoint to Target

Table 5.1 Parameter Values of the Message Passing Protocol for Target

Tracking Performance Evaluation

Table 5.2 Mobile Node Parameter Values for Target Tracking Performance

Evaluation

Table 5.3 Summary of Tracking Performance Comparison for Two

Algorithms

Table 5.4 Energy Model Parameter Values for Analyzing the Effect of

Application Level Energy Consideration

Table 5.5 Parameter Value Changes of the Message Passing Protocol for

Analyzing the Effect of Application Level Energy Consideration

Table 5.6 Summary of Tracking Performance Comparison for Application

Level Energy Consideration

Table 5.7 Mobile Node Parameter Value Changes for Analyzing the Effect of the Cross Layer Power Management

Table 5.8 Parameter Values of the Cross Layer Wakeup Probability

Adjustment Algorithm

Table 5.9 Summary of Tracking Performance Comparison for Three Power Management Scenarios. 


\section{LIST OF FIGURES}

FIGURE

PAGE

Figure 3.1 Approximated Locus of the Target with Sensor Pair Baseline ..................... 42

Figure 3.2 Target Triangulation Using Two Loci.................................................. 43

Figure 3.3 Position Estimation Uncertainty in Target Triangulation ........................... 48

Figure 3.4 Effect of Bearing Angle on Triangulation Error ...................................... 50

Figure 3.5 Effect of Distance from Sensor Pair Midpoint to Target on

Triangulation Error .............................................................................. 51

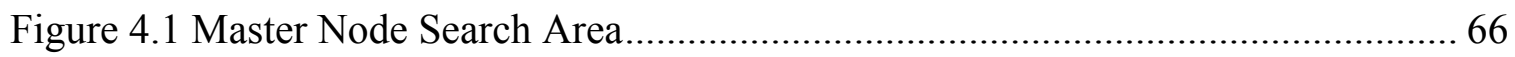

Figure 4.2 The Energy-Efficient and Distributed Self-Organization Algorithm ............. 70

Figure 4.3 Forwarding Path of the Initial Target Location Estimation........................... 73

Figure 4.4 Master Sensor Node Locations............................................................. 77

Figure 4.5 Localization Sensor Group Update ......................................................... 80

Figure 4.6 Opportunistic Sleep/Wakeup Scheme of MAC Protocol .............................. 95

Figure 4.7 Selecting Forwarders for Anycast Based Data Forwarding Scheme............... 97

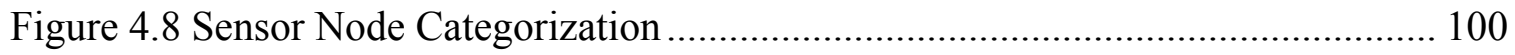

Figure 4.9 Location Based Unicasting Scheme ..................................................... 106

Figure 5.1 Architecture of the Mobile Node Used for the Simulation ......................... 110

Figure 5.2 Overview of the Class Diagram for DSOA Application Agent Implementation................................................................................. 111

Figure 5.3 Architecture of the Mobile Node after Integrating with the Communication Stack 114

Figure 5.4 Mean Target Tracking Error in x Direction for Two Algorithms ................ 121

Figure 5.5 Mean Target Tracking Error in y Direction for Two Algorithms ................ 122

Figure 5.6 Standard Deviation of Target Tracking Error in x Direction for

Two Algorithms 
Figure 5.7 Standard Deviation of Target Tracking Error in y Direction for

Two Algorithms

Figure 5.8 Target Tracking Performance in x Direction without Application

Level Energy Consideration

Figure 5.9 Target Tracking Performance in y Direction without Application

Level Energy Consideration

Figure 5.10 Target Tracking Performance in x Direction with Application

Level Energy Consideration

Figure 5.11 Target Tracking Performance in y Direction with Application

Level Energy Consideration

Figure 5.12 x Direction Tracking Performance for No Sleep-wakeup

Management Scenario

Figure 5.13 y Direction Tracking Performance for No Sleep-wakeup

Management Scenario

Figure 5.14 Sensor Node Energy Availability after Tracking the Second

Target for No Sleep-wakeup Management Scenario.

Figure 5.15 x Direction Tracking Performance for the Fixed Wakeup

Probability Scenario

Figure 5.16 y Direction Tracking Performance for the Fixed Wakeup

Probability Scenario

Figure 5.17 Sensor Node Energy Availability after Tracking the Third

Target for the Fixed Wakeup Probability Scenario. 138

Figure 5.18 x Direction Tracking Performance for the Dynamic Wakeup

Probability Adjustment Scenario.

Figure 5.19 y Direction Tracking Performance for the Dynamic Wakeup

Probability Adjustment Scenario.

Figure 5.20 Sensor Node Energy Availability after Last Target Tracking for the Dynamic Wakeup Probability Adjustment Scenario...

Figure 5.21 A Sample Localization Sensor Groups Selected at Time 11 144

Figure 5.22 A Sample Localization Sensor Groups Selected at Time 29 144

Figure 5.23 A Sample Localization Sensor Groups Selected at Time 42 145 
Figure 5.24 Effect of Sensor Node Density on x Direction Target Tracking

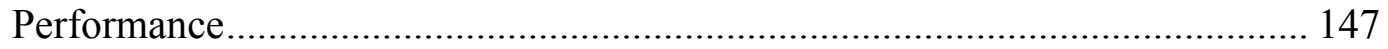

Figure 5.25 Effect of Sensor Node Density on y Direction Target Tracking

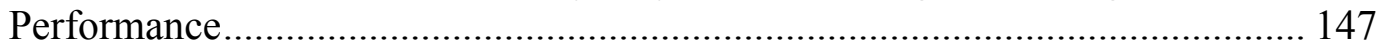




\section{CHAPTER I}

\section{INTRODUCTION}

With the advancements in computing, signal processing and communication technologies, the size and cost of wireless sensor nodes have reduced greatly. Consequently, wireless sensor networks have become popular in a wide range of application areas such as health, military, environment and habitant monitoring [1][2]. Moreover, due to their reliability and low cost, acoustic sensor networks have become attractive in military applications such as ground and underwater target detection, classification and tracking as well as in civilian applications such as environment monitoring [3-5].

\subsection{Background and Motivation}

Acoustic sensor networks have obvious advantages such as extended coverage and improved performance over isolated acoustic sensing devices [3]. In addition, acoustic sensing is completely passive, does not have line-of-sight requirements and acoustic signals are very hard to suppress. Thus, acoustic sensor networks have become popular in battlefield surveillance and situation awareness applications [1][3].

Acoustic source localization strategies are mainly categorized as steered-beamformer based localization strategies, high-resolution spectral-estimation based localization strategies, time difference of arrival (TDOA) information based localization strategies and acoustic energy intensity based source localization strategies [4][6][7]. Another strategy that has gained the attention in recent times is the acoustic energy decay model based localization [8]. In traditional acoustic source localization strategies, acoustic sources are tracked using acoustic sensor arrays built in linear, 
circular or other regular shapes with sensors of the array spatially separated to obtain the desired characteristics in the received acoustic signals [2][3][7][9-12]. The relative geometry of the acoustic sensors is vital for achieving accurate source localization. Thus, maintaining accurate relative positions of the acoustic sensors in the sensor array is very important in these sensor nodes. Therefore, usually the sensor arrays are built on strong and rigid frames to maintain the relative geometry of acoustic sensors. However this restriction significantly reduces the flexibility of the sensor network and makes it infeasible to be deployed in remote or hostile regions through air-drop or other stealth approaches. A simple solution to the above mentioned limitation is to eliminate the presence of acoustic sensor array and develop an acoustic sensor network where each node is equipped with only a single acoustic sensor. This approach allows the flexible and cost effective deployment of sensor nodes through air drop or gun projection to areas that are otherwise inaccessible to human beings. In addition, this also enables the sensor nodes to be very compact, robust and inexpensive. These inexpensive and robust sensor nodes can be densely deployed to form a target tracking sensor network. Moreover, the dynamically formed sensor arrays will provide greater flexibility in the tracking process, compared with the conventional fixed sensor arrays. These features will greatly increase the usability and the flexibility of wireless acoustic sensor networks for target tracking in various environments.

The traditional acoustic sensor nodes with sensor arrays are capable of generating target location measurements themselves. However, the sensor nodes with only a single acoustic sensor at each sensor node cannot perform target localization. Thus, these sensor nodes have to organize themselves into virtual acoustic arrays that can function like 
physical sensor array to perform the target tracking. Achieving this self-organization of sensor nodes is essential for this acoustic sensor network to work effectively. For improved tracking performance, the sensor node selection can be performed based on sensor source geometry to minimize the localization errors. Most of the state of art node selection mechanisms [6][7][9-11] use this approach in the node selection process. In addition, [6] analyzes the major error sources in localization measurement generation. Moreover, as the sensor networks are deployed in ad-hoc manner, the self-organization should be performed in a distributed manner.

Typically, sensor nodes are battery powered and thus have limited power supply. Moreover, once the network is deployed, sensor nodes are physically inaccessible or have very limited accessibility. Thus, replacing or recharging the battery is generally not feasible [1][2][5]. Generally the lifetime of a sensor node is primarily determined by the lifetime of its power supply. Thus, power limitation is a major constraint in sensor network algorithm design, which calls for an effective power management mechanism for an acoustic sensor network to become practical [1-3][5][13]. The major source of power waste in sensor networks is the power consumed by idle listening [13]. The most common method for reducing the idle listening power waste is incorporating a sleep and wakeup management mechanism to the network management protocol. The energy-efficient tracking algorithms proposed in [13][14] and [15] use this approach. In addition to the sleep-wakeup management mechanisms, approaches such as energy aware node selection mechanisms, incorporating of less resource demanding algorithms and employing of energy aware communication mechanisms can be exploited to achieve extended sensor network lifetime. However, the incorporation of power management 
mechanisms could have adverse effects on the tracking performance. In target tracking applications, the goal should be to achieve extended sensor network lifetime while maintaining satisfactory target tracking performance. In addition to the target tracking performance and the energy efficiency, sensor network algorithms should desirably possess characteristics such as scalability, robustness and communication bandwidth efficiency.

\subsection{Problem Statement}

Acoustic sensor networks provide attractive means for tracking ground targets such as tanks and military vehicles. Traditionally, acoustic target tracking is achieved using acoustic sensor arrays at sensor nodes. These sensor arrays are usually rigid shaped and maintaining of the relative positions of these sensors is pivotal for tracking accuracy. This constraint greatly reduces the usability of the sensor network. In order to overcome this limitation, a new acoustic sensor network, which only has a single acoustic sensor at each sensor node, can be used. However, in order to perform as well as traditional sensor arrays, the sensor nodes have to self-organize themselves into virtual sensor arrays to perform target tracking. In this research, we study the self-organization problem and propose an energy-efficient distributed self-organization algorithm for ground target tracking using acoustic sensors with only a single sensor at each sensor node. Inspired by the sensor source geometry based node selection mechanisms studied in [6][7][9][10] and [11], we develop a node selection criterion to minimize the acoustic source localization error. The distributed self-organization algorithm performs the node selection based on the developed node selection criterion and target tracking. As the node selection is based 
on the sensor source geometry, the self-organization algorithm updates the selected sensor group to match the dynamics of the target.

Energy efficiency is a major constraint in designing algorithms for resource limited sensor networks. The energy efficiency of the sensor network management protocol is studied and an energy-efficient and low latency medium access control (MAC) protocol with location based data forwarding mechanism [16][17] is incorporated into the tracking algorithm to support efficient power management. Generally, sensor networks are densely deployed and the interesting events such as presence of a target are rare in sensor networks. In addition, the sensing and communication requirements of the sensor networks are closely attached to these interesting events and are thus temporally and geographically related to these interesting events [1][2][13]. Efficient power management in sensor networks requires this event information as well as the network status and the successful estimation of the future communication requirements of the sensor network. However, all above information is not available in a single network layer. Therefore, effectiveness of cross-layer design in achieving the tradeoff between energy efficiency and the target tracking performance is studied and a cross-layer power management algorithm, which combines the event information from the application layer and network conditions from the MAC layer, is proposed.

The scope of this research is limited to the tracking of a single ground target. In addition, it is assumed that the sensor nodes know their own locations and the sensor nodes are time synchronized. The sensor node self-localization and time synchronization are research areas of their own and are beyond the scope of this research. 


\subsection{Research Objective}

The primary objective of this research is to design an energy-efficient distributed self-organization algorithm to manage sensor nodes for the new wireless acoustic sensor network for ground target tracking. The developed algorithm will use only a single acoustic sensor in each sensor node rather than the traditional approach of using sensor arrays in the sensor nodes. The developed algorithm should be able to perform the distributed collaborations between the nodes of the network to perform target tracking. In addition, the proposed algorithm should be able to achieve effective tradeoff between target tracking quality and the energy efficiency in order to achieve extended network lifetime while maintaining satisfactory near real-time target tracking performance.

\subsection{Contributions}

Acoustic sensor networks are becoming more and more popular for target tracking in military applications such as battlefield surveillance and for civilian applications such as surveillance and habitant monitoring. Our contribution is the development of an energy-efficient distributed self-organization algorithm for ground target tracking using acoustic sensor networks with only a single acoustic sensor at each sensor node.

- The distributed self-organization algorithm: A new type of sensor network with only a single acoustic sensor at each sensor node, which uses the bearing measurements for target localization, is employed for the target tracking. The distributed self-organization algorithm dynamically selects a localization sensor group to perform the target tracking. The localization sensor group selection is performed to minimize the localization error based on the sensor source geometry. The localization 
sensor group is dynamically updated to match the target dynamics. A two-step node selection process is employed to minimize the complexity of the node selection process.

- Cross-layer power management: At the application level, energy considerations are incorporated into the node selection process for effective power management and the two-step node selection process greatly reduces the communication requirements of the algorithm resulting in improved energy efficiency. In addition, a location based message forwarding mechanism is utilized to reduce the communication complexity in localization sensor group selection and update processes. Most importantly, a cross-layer wakeup probability adjustment algorithm, which combines event information from the application layer and the network condition from the MAC layer, is employed to achieve extended sensor network lifetime.

\subsection{Dissertation Organization}

The rest of this dissertation is organized as follows: Chapter II introduces theoretical background of acoustic source localization and related work on energy-efficient target tracking and energy efficiency of sensor networks. Chapter III details the target localization and tracking methods employed in the proposed self-organization algorithm. The basis for the node selection criterion for the proposed self-organization algorithm is discussed in this chapter. The proposed energy-efficient and distributed self-organization algorithm is introduced in Chapter IV. This chapter includes a detailed discussion on the algorithm and the message passing protocol developed to accomplish the algorithm in a distributed manner. In addition, the incorporation of energy conservation to the developed self-organization algorithm is 
discussed in details in this chapter. This discussion includes details on the energy-efficient and delay-sensitive MAC protocol [16][17] that is incorporated to the tracking algorithm and the novel cross layer wakeup probability adjustment scheme developed for efficient power management. Chapter V elaborates the implementation of the proposed self-organization algorithm in network simulator (ns-2, [18]) and the performance evaluation of the algorithm. The final conclusions and future research directions are presented in Chapter VI. 


\section{CHAPTER II}

\section{BACKGROUND AND RELATED WORK}

In this chapter we introduce the background and the literature related to our research. The first section provides an introduction to the acoustic source localization strategies proposed in literature. In the second section, we provide a detailed literature review on energy-efficient target tracking in wireless sensor networks.

\subsection{Acoustic Source Localization Strategies}

Several coherent and non-coherent acoustic source localization strategies are proposed in literature such as the (correlation driven) time difference of arrival (TDOA) based localization strategy, the high-resolution spectral estimation-based localization strategy, the steered-beamformer based localization strategy and the received signal energy measurement based strategy [6-8][19-27]. This section provides an overview of these different strategies.

In high-resolution spectral estimation-based localization strategy, the TDOA of acoustic signal at two acoustic sensors are estimated using the phase information of the cross-power spectrum of the received acoustic signals [6][7]. In contrast, the (correlation driven) TDOA based localization strategies estimate the TDOA value only using the correlation methods [6][7][20]. The direction of arrival (DOA) of the acoustic signal can be estimated with reference to the sensor locations using the calculated TDOA value. Conversely, beamformer based localization strategies calculate the DOA of acoustic signal directly using filtered, weighted and summed version of the received acoustic signals $[6][22][23]$. Once the TDOA or DOA information is available, locus of the acoustic source relative to the sensor nodes can be estimated using this information. 
Multiple such loci are required for the acoustic source localization. The acoustic energy based localization strategies mostly employ the acoustic energy decay model based (distance) range estimation for localization [8]. In this approach, the intersection of the geographic ranges of multiple sensors (determined using the estimated distance range) is used for localization. However, some acoustic energy based strategies estimate the DOA of acoustic signal using the acoustic intensity information at different sensors and perform the localization using this DOA estimation [7].

An acoustic energy decay model based acoustic source localization method that employs maximum likelihood estimation with expectation maximization was proposed by Sheng and $\mathrm{Hu}[8]$. Maximum likelihood estimation and least-squares estimation are widely used with beamforming for the source localization [21]. Chen et al. [22] have proposed a maximum likelihood source localization method for near field source localization. In [23], this method is extended to far field source localization. Yao et al. [25] introduced a blind beamforming technique that can be applied to source localization using randomly distributed placement unknown sensors. A beamformer based localization method within the particle filtering framework was proposed by Ward and Williamson [27]. Brandstein et al. [6][26], proposed framework for speech source localization using the TDOA information based localization strategy. They have proposed linear intersection algorithm, a closed form source localization algorithm using TDOA measurements.

The received acoustic signal strength based strategies are comparatively simple and generally requires less communication and processing resources [7][8]. However, the localization accuracy of the energy based estimation strategies can degrade specially in 
far field target localization [7][20][23]. The acoustic signal intensity based strategies require precise phase and amplitude matching of microphones and also are highly susceptible to the environment conditions [7]. The steered beamformer based localization strategies usually provide high quality localization performances, however accuracy of the method depends on the knowledge of spectral contents of the acoustic signal and the noise, which are typically unavailable [6]. In addition, they usually require costly antenna arrays [8]. Moreover, the computational demands of the strategy are very high and the computational optimizations available are highly ineffective for these strategies [6]. High-resolution spectral estimation-based localization strategies require the spatiospectral correlation matrix derived using the received acoustic signals. Thus, they are highly susceptible to the accuracy of modeling the sensor modeling errors [6]. In addition, they are highly sensitive to noise [7]. Robust variations of the spectral estimations are proposed in the literature, however they are computationally high demanding [7], especially for resource limited wireless sensor networks. Moreover, for wideband signals the computational complexity of the high-resolution spectral estimation-based localization strategies is high [6]. Although TDOA based strategies are resource demanding compared to the energy based localization strategies, they are more robust compared with energy based localization [6][7]. Compared with steered beamformer based strategies and spectral estimation-based localization strategies, the TDOA based strategies are less resource demanding [6][7][20]. In addition, although TDOA based strategies are not as robust as steered beamformer based strategies, they provide satisfactory localization performance with great simplicity and resource efficiency. Moreover, TDOA based strategies can be extended to track multiple targets [7]. 


\subsection{Related Work}

This section provides a detailed discussion on energy-efficient target tracking using wireless sensor networks. We first discuss the target tracking algorithms proposed for wireless sensor networks, primarily focusing on acoustic target tracking. The second part of the discussion investigates energy-efficient target tracking algorithms proposed for wireless sensor networks. In addition to the application level power management, there are several networking level power management methods proposed for wireless sensor networks. The third part discusses the networking level power management strategies for wireless sensor networks with emphasis on power management for target tracking applications.

\subsubsection{Target Tracking Using Wireless Sensor Networks}

Target tracking using acoustic signals has been used from very early times as far back as World War II. With the emergence of tiny sensor nodes with multi dimensional sensors and powerful communication and computing capabilities, target tracking using wireless sensor networks has gained wide attention from researchers. As a result, many algorithms have been proposed for target tracking using wireless sensor networks.

A self-organizing protocol for energy efficient target tracking is proposed by Biswas and Phoha [28]. In their approach, the sensor network self-organization is performed through a four-phase self-organization protocol, which consists of self-awareness, self-reconfiguration, self-repositioning, and self-adaptation phases. These four phases of the protocol enable the sensor nodes to initially organize and then reconfigure into an energy-wise optimal subset of active nodes for target tracking. Energy conservation is achieved by switching off the redundant nodes, which have overlapping 
coverage. A dynamic space time clustering algorithm [29] is used for target tracking. The algorithm performs the node clustering based on closest point of approach (CPA) information. Received acoustic signal strengths are used to detect CPA of a target to a sensor node. Then, the CPA information from itself and neighboring nodes collected in a predefined time window is used for the cluster formation and target tracking.

Ekman et al. have proposed a ground target tracking algorithm using acoustic sensors [30] [31]. The algorithm employs particle filters and statistical data association for target tracking using bearing measurements obtained from acoustic sensors. In this algorithm, solution to the target tracking problem is given within the Bayesian recursive framework, where the state estimations are obtained using the particle filters. The particle filter is based on the classical sampling importance resampling (SIR) scheme [30]. However, it is redesigned to support multiple target tracking. Sequential Monte Carlo techniques are used to represent the solution to particle filter using samples, which reduces the complexity of the solution. The combination of information from sensor nodes is performed using a modified version of sample based joint probabilistic data association (JPDA) scheme. Here, the particle weights of the filter are updated using the posterior probabilities to provide more realistic particle weights that match the tracking environment. Moreover, their algorithm also supports handling of constraints on motion pattern of target such as terrain constraints and velocity constraints by incorporating them into the particle filter.

A cluster based acoustic target tracking system is proposed by Wang et al. [32] for tracking impulsive acoustic targets with known acoustic signatures. The system architecture consists of two main self-contained subsystems, the acoustic target tracking 
subsystem and the communication subsystem. The acoustic target tracking subsystem is responsible for detection and triangulation of the acoustic signal. Sensor nodes are organized into clusters and clusters are the primary units for localization and tracking. A cluster consists of a cluster head and slave sensor nodes, and each sensor node in the cluster collaborates in sensing and communication to perform the tracking. When an acoustic signal with known signature is received, slave nodes report the signal and the timing information to the cluster head and the cluster head performs the localization and reports the results to the data sink. The communication subsystem is responsible for reporting the tracking results to the data sink. In order to achieve effective bandwidth utilization and low latency, they have proposed a quality-driven redundancy suppression and contention resolution scheme and a multi-parent sink tree routing scheme.

Sheng and $\mathrm{Hu}$ [33] have proposed a self-organization algorithm for distributive moving target tracking. They have used energy based source localization [8] with the maximum likelihood multiple-target localization [34] for the target localization in their tracking algorithm. In their scheme, the sensor nodes that receive signal from the same target dynamically form a cluster for target tracking. Sensor nodes within the cluster are further organized into cliques with each clique having a master sensor node and several slave nodes. The communication within the clique is achieved using broadcast messages and the master nodes communicate with each other in peer to peer fashion. The self-organization algorithm performs non-overlapping clustering of sensor nodes in a distributed manner. For this, each sensor node decides which target tracking it should perform depending on a cost function value. The cost function evaluates the information gain it can provide for each target tracking. 
Chen et al. [35] proposed a decentralized dynamic clustering algorithm for single target tracking. A hierarchical sensor network is employed for target tracking in this approach. The hierarchical sensor network consists of a static backbone of sparsely placed high-capability sensor nodes and densely deployed low end sensor nodes. The high-capability sensor nodes become the cluster heads and the low end sensor nodes become slave nodes in the cluster formation. Target localization is performed using energy based acoustic source localization. For the cluster head election, a probabilistic leader volunteering procedure is employed with the use of Voronoi diagram [36]. In this approach, each sensor node calculates the probability of the node being closest to the target, using the known relative sensor node positions and the target distance estimation. This probability is used as the backoff timer for the leader volunteering by high-capability sensor nodes. The cluster heads send out an information solicitation packet when they detect a target, and the slave nodes join the cluster depending on the probability of it being the closest to the target, which is detailed earlier.

Extended Kalman filter based target tracking mechanism using bearing only measurements is proposed by Kaplan and Le [37]. The bearing measurements are obtained using a circular microphone array with nine microphones at each sensor node. A preparation compensated nonlinear least-square localization strategy is developed to perform the target localization using the bearing measurements from several sensor nodes.

A distributed two-tier target tracking algorithm is proposed by Shi et al. [38]. The algorithm is mainly focused on tracking using linearly placed sensor nodes. Energy based acoustic source localization is employed for localization. In this two-tier approach, a 
rough estimation is obtained in the first tier using the Viterbi algorithm [39] based multiple hypothesis tracking algorithm. In the second tier, a set of sensor nodes which are located closer to the target is selected, and maximum likelihood estimation [34] is performed using the measurements from the selected sensor nodes to obtain accurate target location estimation.

A target tracking algorithm using joint acoustic and video sensors is presented by Cevher et al. [40]. A particle filter that is capable of handling multiple modalities is proposed and a time-delay variable is incorporated to handle the acoustic-video data synchronization. Yu et al. [41] proposed a neural network aided Unscented Kalman filter for target tracking. In this approach, the nonlinear filtering is achieved using the Unscented Kalman filter and the neural network is incorporated to correct the modeling errors caused by target maneuvering. A framework for collaborative signal processing in distributed sensor networks for target detection, classification and tracking is proposed by Li et al. [42]. This collaborative signal processing framework incorporates goal-oriented on demand distributed processing, information fusion, and multi-resolution processing capabilities. In addition, the framework is integrated with a location centric networking/routing algorithm [43]. A target tracking algorithm based on mobile agent paradigm is developed by Tseng et al. [44]. In this scheme, a master agent performs the tracking with the support of two slave agents. The master agent always stays in the sensor node that is closest to the target, and dynamically selects the slave agents. The mobile agent prototypes were developed using IEEE $802.11 \mathrm{bNIC}$. 


\subsubsection{Energy Efficient Target Tracking Using Wireless Sensor Networks}

Power limitation is a major design constraint in developing algorithms for wireless sensor networks [1][12-15]. Thus, achieving energy efficiency has been a major goal in target tracking algorithm design for wireless sensor networks. Several approaches have been used for achieving energy efficiency in target tracking algorithms. Some of the popular techniques proposed in literature are, employing less power demanding processing mechanisms, reducing the communication requirements of the algorithms, incorporating energy considerations into node selection, performing data aggregation, using of energy aware routing and MAC layer protocols, and employing sleep-wakeup management schemes [1][12-15][45]. This section provides a detailed investigation on energy-efficient target tracking algorithms for wireless sensor networks.

An energy-efficient cross layer architecture for acoustic target tracking using wireless sensor networks is proposed by Song and Hatzinakos [13]. They proposed a lightweight acoustic energy based target localization algorithm. Specifically, the target location is estimated as an optimal linear combination of the location information of participating sensor nodes. A sleep-wakeup scheme is employed for the efficient power management. In their model, two separate radios are used for node wakeup management (wakeup radio) and communication (primary radio). A cross layer architecture, where application layer and MAC layer interact for high protocol efficiency, is proposed. Initially, the first node that detects the target is selected as the leader node. Then, this leader node awakens and notifies all its neighboring nodes to sense the target by sending a message through the wakeup radio. After sensing the target acoustic signal for some time, each sensor node calculates a utility function based on the received signal strength. 
The sensor node with the highest utility is selected as the new leader node. A subset of nodes that sensed the target, sends their utility function value and location information to the new leader node and the new leader node performs the localization. The number of nodes that participates in the localization is determined by the new leader depending on the quality of service, which is calculated using the improvement in localization accuracy. Moreover, the previous leader node sends the track history to the new leader node, and the new leader node generates track information using history and the calculated location estimation. As the nodes that do not receive a notification from a head node do not have to participate in tracking, they can turn off their sensing unit and the radio for communication unit and transform to the sleep state to conserve power.

VigilNet, implementation of a large scale energy-efficient sensor network for detection, classification and tracking of targets is presented in [46-49]. In VigilNet, the groups of sensors that detect the target cooperate to track the target. The deadline partition method is employed to enforce the real-time target tracking. In addition, the energy conservation is achieved by employing a periodic sleep-wakeup management scheme. In this approach, a set of nodes is selected as sentry nodes and is kept awake for the monitoring of events. The other nodes are allowed to sleep until an interesting event is detected by these sentry nodes. In case of detecting an interesting event, the sentry nodes awaken other nodes in the region to perform monitoring. The tradeoff between energy consumption and the tracking quality is achieved by adjusting the backoff delay employed in sanity node selection, which will adjust the sensitivity of the sensor network.

The energy-quality tradeoffs of target tracking using wireless sensor networks are studied by Gui and Mohapatra [14], and a quality of service metric is proposed to 
evaluate the level of surveillance of the system. Moreover, a sleep-wakeup management scheme that operates in the surveillance state as well as in tracking state is proposed for improved energy-efficiency. In the surveillance state, a neighborhood cooperative sleeping based sleep plan for each sensor node is proposed. This schedule is an extension of Probing Environment and Adaptive Sleeping (PEAS, [50]) algorithm and is termed Probing Environment and Collaborating Adaptive Sleeping (PECAS). In this approach, each node periodically awakens and probes for active nodes. If no active node is found it stays awake for one time duration. If an active node is present, that active node will reply to the probe with the end time of its active duration. Then depending on the replies received by the probing node, it selects its sleep duration depending on the collected end times and enters sleep state. For the tracking state, a collaborative proactive messaging scheme that wakes up and shuts down the sensor nodes with spatial and temporal preciseness is proposed. In this scheme termed proactive wakeup, the sensor nodes that overhear the tracking packets but cannot sense the target keep awake expecting the target to enter its sensing region in the near future. Thus, the combination of two schemes for the two states of the sensor nodes are employed to achieve efficient power conservation through sleep-wakeup management.

$\mathrm{Xu}$ and Lee [51] proposed a localized prediction based energy-efficient tracking algorithm. A hierarchical cluster architecture is employed for the network and a localized prediction termed duel prediction is used for the estimation and prediction of target movements. The sensor field is partitioned into non-overlapping logical cells, which are defined as areas around the sensor nodes. Each cell contains exactly one node and if the target is located within that cell, the tracking is performed by that particular node. In this 
duel prediction method, the sensor node that is responsible for tracking the target as well as the corresponding cluster head generates target state predictions using the target movement information of the previous time intervals. In addition, the sensor node responsible for the tracking will estimate the target location using the measurements from current time interval. Then, it will compare the prediction and the estimated locations and if the prediction is not accurate according to the estimate (obtained from current measurements), the updated estimation will be sent to the cluster head. As most of the nodes in the network are not participating in the tracking process, they enter sleep state and conserve energy. The tracking node awakens the sensor nodes that are in the predicted path of the target for continuation of tracking.

Jiang et al. [52] proposed a target moving direction based sleep scheduling algorithm for target tracking applications. In their approach, initially all the nodes are in the surveillance mode. In this mode, all the nodes follow a random and asynchronous sleep pattern with a fixed duty cycle. Once a target is detected, tracking is performed by the collaboration of sensor nodes in the tracking subarea with one node selected as the root node. The root node broadcasts an alert message with target state information to awaken the nodes in the next tracking subarea depending on the prediction of target path. For target path prediction, a direction-based tracking contour deciding mechanism is proposed. In [53], this work is extended and sleep scheduling algorithm for multiple target tracking (SSMTT) is proposed. In tracking multiple targets, when the paths of targets interfere with each other, some nodes may be eligible to track multiple targets. In such scenarios, the nodes need to be awakened only once and the duplicate wakeup broadcast messages can be eliminated to save power. SSMTT exploits this and eliminates 
the wakeup broadcast messages or reduces the transmission power of wakeup broadcast messages in such scenarios, for improving power conservation.

A cluster based dynamic energy management algorithm is proposed by Wang et al. [54] for tracking acoustic targets. They have employed direction of arrival (DOA) based target localization and a particle filter for target position prediction. In addition, a dynamic adaptive clustering scheme, which uses localized broadcasts for distributed cluster head election is proposed and combined with LEACH (Low-Energy Adaptive Clustering Hierarchy) [55] for the cluster generation. Moreover, an optimal intra-cluster routing mechanism is developed based on the Dijkstra's algorithm. A sleep-wakeup management scheme is employed for the efficient power management. In this scheme, when there is no sensing or communication requirement, the sensor nodes follow a sleep-wakeup pattern with a predefined duty cycle. However, in contrast to other sleep-wakeup schemes, in this approach, even the sensors that participate in tracking and communication tasks also follow the sleep-wakeup pattern. More specifically, the sensors that perform tracking or data forwarding tasks stay awake for the current interval and then transform to sleep state. However, unlike previous case, the time interval of sleeping is decided depending on the target state estimation obtained using the particle filter. As the individual sensor nodes perform the awakening using their own timers, there is no requirement for wakeup message broadcasting in this mechanism. In addition, a discrete binary particle swarm optimization based selection mechanism is proposed for the selection of tracking sensor node group from available active sensor nodes. This method provides further energy conservation in collaborative sensing and data forwarding for target tracking. 
Energy-efficient collaborative target tracking algorithm using cost-reference particle filtering proposed by $\mathrm{Yu}$ et al. [56], too uses a cluster based approach to target tracking. In this approach, only one cluster is responsible for tracking the target at a given time and the target localization is performed using energy based target localization. For target tracking, a set of selected cluster members that lie close to the predicted target location will collect the acoustic energy measurements and send them to the cluster head. The cluster head is responsible for selecting the set of cluster members, gathering measurements from them, estimating and predicting the target state, sending tracking information to base station, and passing the sensing and tracking operation to the next cluster. A new class of particle filter termed cost-reference particle filter (CRPF) is proposed for estimating and predicting the target location. In this CRPF, a user defined cost function is employed for evaluating the quality of target state. In addition, to balance the energy consumption at sensor nodes, an event-driven cluster reforming scheme is proposed. The cluster reforming process rotates the cluster head after each target tracking. Moreover, efficient power management is obtained by putting the idling clusters to sleep. When the currently active cluster head determines that target is moving away from it and is nearing the cluster boundary, it will send an activation message to an appropriate neighboring cluster head with current target state estimations and required algorithm parameters. Then, the new cluster head will notify its members to start sensing the target.

RARE, an energy efficient target tracking protocol is proposed by Olule et al. [57]. Two algorithms to achieve effective power management through reducing the number of nodes participating in the tracking process are proposed. First, the RARE-Area 
algorithm reduces the number of tracking nodes by selecting only the sensor nodes that receive sensing data of a given quality. Then, the RARE-Node algorithm acts upon the set of selected nodes and makes sure that the nodes with redundant information do not participate in the tracking process. These two steps ensure that only a subset of nodes that can provide quality information, participate in the tracking process, which enables the power conservation.

Cao et al. [58] proposed a near-optimal sleep scheduling algorithm for energy-efficient sensor-network-based surveillance applications. In this work, they have proposed a two-level sleep scheduling scheme. In the first level, a minimal set of sensor nodes are selected to maintain the sensing coverage. This set is called the primary subset, and the other nodes in the network are put into sleep. This process is repeated with large duty cycles. In the second level, the nodes that belong to the primary subset are sleep scheduled in duty cycles at a frequency higher than the first level. In addition, they have proposed an iterative duty cycle adjustment scheme, which achieves the local optimum for a given detection delay. Moreover, a connectivity maintenance protocol is proposed to minimize the packet delivery delay.

Abrams et al. [59] proposed a set K cover energy-efficient monitoring algorithm for wireless sensor networks. In this approach, the sensor nodes are divided into covers and the covers are activated in round robin fashion to maintain the monitoring, while the other nodes are put asleep to achieve effective power conservation. Moreover, three algorithms are proposed for generating the covers. In the first algorithm, each sensor node assigns itself to a cover randomly. In the second distributed greedy algorithm, each sensor node assigns itself to a cover based on neighboring information and the current 
state of the covers. The selection of cover is targeted at maximizing the coverage of the cover, which has the minimal local coverage. The third centralized greedy algorithm, which depends on the global information, assigns the sensor nodes into the covers to maximize the coverage of each cover.

An energy-efficient dynamic convoy tree-based collaboration framework is proposed for target tracking by Zhang and Cao [60]. The framework constructs the initial convey tree for target tracking when a target is first detected, and then updates the tree to match the target movements. When a target is first detected, sensor nodes that detect the target collaborate to select a root node and construct the initial convoy tree. The root node then collects target information from the sensor nodes in the tree and obtains a refined target state estimation. As the target moves, the tree is dynamically updated by removing the sensor nodes that are far away from the target and adding the sensor nodes that are close to the target. Two energy-efficient methods, namely conservative scheme and the prediction-based scheme are proposed for the convoy tree updating. When the convoy tree is updated, the tree is reconfigured to update the root node. Two tree reconfiguration schemes, the sequential reconfiguration and the localized reconfiguration are developed for energy-efficient reconfiguration of the updated convoy tree. In addition, for effective power management, the geographical adaptive fidelity (GAF) protocol [61] is incorporated to the framework. In this power management scheme, the sensor network is divided into grids and each grid contains a grid head. When a target is not present, only the grid head is kept awake, while the other nodes in the grid wakeup periodically. The root node of the convoy tree activates the sensor nodes in the target moving direction by notifying the grid heads. 
Yang et al. [62] proposed an energy efficient distributed target tracking algorithm called Predict-and-Mesh. A prediction based node awakening scheme with a mesh process for recovering from prediction failures is used in this algorithm. Initially, the sensor nodes are in sleep mode and are awakened depending on the prediction of the target movement. Two prediction models namely, $n$-step prediction and collaborative prediction are utilized for the target movement prediction. In $n$-step prediction, the same set of nodes predicts the target for several steps and for collaborative prediction, the prediction information from previous set of nodes is passed onto the next set of nodes. The proposed mesh process enables recovering from prediction failures by awakening the surrounding nodes to form a mesh around the target. The energy-efficiency is achieved through the employed sleep-wakeup scheme.

An energy efficient multiple target tracking scheme is proposed by Yeow et al. [63]. They have used cluster based network architecture, and power savings are achieved by making the non tracking nodes sleep and by minimizing the workload of the tracking nodes. In this scheme, the sensor nodes are dynamically managed using the spatial information obtained by predicting a target's trajectory through experience. Target trajectory model is assumed to be Gauss-Markov mobility model. Moreover, the tracking problem is formulated as a hierarchical Markov decision process (HMDP) and is solved through neurodynamic programming. In addition, the sensing rate is dynamically adjusted depending on the estimation accuracy to further improve the power conservation.

Chu et al. [12] proposed an energy-efficient sensor querying technique and a novel routing mechanism for sensor network applications. The key idea of their work is 
utilizing an information utility measure for sensor node selection and dynamically guided data routing. In the sensor querying technique termed information-driven sensor querying (IDSQ), the nodes that can contribute to maximize the information gain are selected. They have used a cluster based approach in node selection and the cluster head selects the set of sensor nodes that can maximize the information gain and queries them. For routing the query and the reply packets, a generalization of directed diffusion routing, constrained anisotropic diffusion routing (CADR) is proposed. In this routing algorithm, the information gain and communication cost are considered in dynamic route formation. In [64], the IDSQ approach is formulated for target tracking applications. Extending the IDSQ approach to acoustic energy based localization, and acoustic beamformer based localization are studied in [65].

A novel dynamic grid-based tracking scheme for tracking mobile targets in energy-efficient manner is proposed by Zhou and Sen [66]. In their approach, they have formulated the target tracking problem as a problem of finding the sequence of optimal aggregation trees that minimize the energy consumption. In order to achieve this in a distributed manner, a virtual grid structure is employed. The sensor network field is divided into a grid structure and the nodes closest to the grid points are selected as the grid nodes. Then, each node selects the grid node within its neighborhood, which is closest to the target, as the aggregation node. Target localization is performed using the received signal strength measurements shared by the neighboring nodes. Once the localization is complete, the target location information is forwarded to the aggregation node and then to the sink. This data aggregation results in reduced communication overhead, which enables achieving energy-efficiency. 
Oh et al. [67] proposed a scalable and hierarchical multiple target tracking algorithm based on Markov chain Monte Carlo data association [68]. The hierarchical sensor network consists of few super nodes with higher capabilities and average nodes. Each average sensor node is assigned to a tracking group headed by a super node. When a super node detects a target, it performs the tracking using the information from average nodes in its tracking group. The information from super nodes is combined in a hierarchical manner to achieve energy efficiency. A localized clustering algorithm and a direction diffusion based localization mechanism are proposed by Estrin et al. [69]. The clustering algorithm is capable of performing scalable hierarchical cluster architecture, which will result in reduced power consumption.

A distributed, energy-efficient and light-weight framework for target tracking using wireless sensor networks is proposed by Lee et al. [70]. They have employed energy based acoustic source localization strategy with a novel localization algorithm, called Ratiometric Vector Iteration (RVI). This algorithm provides localization estimations based on distance ratio estimates from the sensed signal strength ratios. The localization is performed using measurements from three sensors and is iteratively updated until a satisfactory estimation is obtained. A received signal strength based backoff timer is employed for selecting the localizing nodes and the leader node. The leader node performs the localization using RVI algorithm and reports the estimations to the subscriber. In addition, the reporting frequency is dynamically adjusted considering the target's movement to achieve energy-efficiency while maintaining the tracking quality. 
Yu et al. [71] proposed a quality aware information collection framework for target tracking using wireless sensor networks. However, their approach is centralized and the sensor nodes are controlled by a central server. Energy based localization is employed for localization and localization is performed at the server. Importantly, the sensor nodes are dynamically controlled by the server depending on the tracking quality and the required tracking quality to achieve the energy conservation. A power control mechanism to achieve an optimal trade-off between target tracking accuracy and the power efficiency is proposed by Wagner and Cristescu [72]. In this approach, a hidden Markov model is employed for the target movement. The problem of achieving the energy-quality trade-off is modeled as an optimal control problem with partial observations of target location, and a hand-off technique is proposed to achieve energy efficiency.

\subsubsection{Energy Efficiency in Wireless Sensor Networks}

The power management methods discussed in the previous subsection are mainly targeted at target tracking wireless sensor networks. They are primarily application level power management protocols that may use the support from the networking layers (lying below) for effective power management. In addition to the power conservation methods employed by the target tracking applications, there are several energy-efficient networking protocols that are proposed for wireless sensor networks. These networking level power management approaches can provide power management on their own as well as can be incorporated for wireless sensor network applications for improved cross layer power management. Several such network layer and medium access control (MAC) layer protocols are discussed here. 


\subsubsection{Energy Efficiency in Network Layer}

ASCENT, an adaptive self-configuring sensor network topology for efficient power management is proposed by Cerpa and Estrin [73]. ASCENT incorporates a sleep-wakeup management scheme for power conservation. The algorithm adaptively selects a set of nodes to be active nodes to perform multi-hop packet routing. The other nodes enter sleep state and periodically check whether they should become active. More precisely, the sensor nodes in the network can be on one of four states, namely active, passive, checking and sleeping. The nodes in the passive state after some time transfer to sleep state, and after sleeping for some time transfer back to passive state. When an active node detects a higher packet loss rate, it sends out a help message, requesting neighboring nodes to become active. When a neighboring node in passive state receives this message, it transfers to the checking state. The nodes in checking state probe the communication for some time and decide whether they can help to improve the communication. If they decide that they can improve quality of communication, they become active. Otherwise they go back to the passive state. If the node becomes active, it sends out a message to announce its presence and participates in communication. Thus, the individual sensor nodes locally self-configure their states so that the communication quality is maintained while the effective power management is achieved.

Greunen et al. [74] proposed an adaptive sleep discipline for energy conservation in wireless sensor networks. This approach allows the sensor nodes to sleep while maintaining the performance requirements of the application. In this approach, each node independently decides when to sleep and wakeup based on its local information. When a sensor node in sleep state becomes active, it announces to the neighboring nodes that it is 
ready to forward packets. Then, it stays awake till all the waiting packets are received. In addition, depending on the number of packets it received and the longest waiting time of the packets, it dynamically adjusts its maximum sleep time to match the application requirements. After this, it forwards the received packets and goes to sleep for a random time period, which is within the adjusted maximum sleep time. Moreover, an opportunistic routing mechanism is employed for timely delivery of packets. This mechanism is a modification of geographic routing where the sender forwards the packets to one of the available nodes from a set of equivalent node set rather than forwarding the packets to a fixed node. The equivalent node set consists of the nodes that are suitable for forwarding the packet to the destination.

Geographical adaptive fidelity (GAF), an energy-efficient protocol for wireless sensor networks is proposed by $\mathrm{Xu}$ et al. [61]. GAF is an add-on to ad-hoc routing protocols which can support the routing algorithm to attain higher energy efficiency. The GAF algorithm identifies the nodes that are equivalent from the routing perspective and makes the redundant nodes sleep to achieve extended network lifetime. In addition, the algorithm maintains a constant routing fidelity, which can be determined based on application requirements. The nodes can be in one of three states, active, sleeping or discovery. Initially all the nodes are in discovery state. At this state, nodes send out a discovery message and wait for some time. Then, the node transfers into active state and participates in routing activities. After spending some time on active state, the node goes back to the discovery state and sends out a discovery message again. The node enters the sleep state (from active or discovering states) if it finds out that there is a better (higher ranked) equivalent node that can handle the routing. The node equivalence is determined 
using the node location and a virtual grid, where the nodes that belong to same grid are considered equivalent. The nodes are ranked using a scheme that can be specified by the application, and a simple ranking method would be to rank based on the energy left in the nodes. A node in the sleeping state enters the discovery state after sleeping some time. The sleep time and the active time of the protocol can be adaptively adjusted by the application depending on its requirements, thus enabling the adaptive fidelity.

SPAN, an energy-efficient coordination algorithm for topology maintenance in ad hoc wireless networks is proposed by Chen et al. [75]. Contrasting to GAF [61], SPAN is placed below the routing layer and on top of MAC layer and is a proactive protocol. However, similar to GAF [61], SPAN also makes the nodes sleep periodically for extended network lifetime. In SPAN, each node locally decides whether to sleep or participate in routing, depending on how many nodes it can benefit and the amount of energy left in the node. The number of nodes it can benefit is measured by the number of nodes it can connect. The nodes that are sleeping periodically check whether there are any nodes that are not connected in its neighborhood and decide to participate in the routing process. Each node that participates in routing, periodically checks whether the nodes, which are connected through it can maintain the connectivity without its service. If it is possible, the node decides to sleep. In addition, after participating in the routing process for some time, a node will decide to sleep if there are other nodes that can replace its role.

Sparse Topology and Energy Management (STEM), which enables effective power management through sleep-wakeup is proposed by Schurgers et al. [76]. In contrast to sleep-wakeup management mechanism discussed above that exploits the high 
node density of sensor network, this protocol provides energy conservation at the cost of increased latency. In this approach, two separate radio communication links, a low power consuming link for wakeup management and a regular link for communication are used. The radio link for communication is always kept off except when transmitting or receiving. The low power link follows a periodic sleep-wakeup pattern. When a node wants to send data, it will send a beacon packet to the destination through the wakeup management link and establish a connection. Then, the data is sent through the communication link. In addition, the STEM protocol can be combined with protocols such as GAF [61] and SPAN [75] to further improve the power conservation.

Zheng et al. [77] proposed asynchronous wakeup for effective power management in wireless sensor networks. They derived an optimal wakeup schedule and a neighbour discovery and schedule bookkeeping protocol. This protocol operates on the proposed optimal wakeup schedule to support effective power management.

A balanced-energy sleep scheduling mechanism intended to achieve a reduced energy consumption as well as balanced energy consumption over the network is proposed by Deng et al. [78]. A static cluster based sensor network is employed and a cluster head managed sleep-wakeup management mechanism is proposed for the power conservation. The cluster head dynamically selects a set of sensor nodes to sleep for power conservation. The node selection is performed based on the distance from the sensor node to the cluster head and is expected to maximize the network lifetime by minimizing the energy consumption as well as balancing the energy consumption among nodes in the cluster. 
Xing et al. [79] proposed minimum power configuration (MPC) approach, a cross layer approach to the power conservation in wireless sensor networks. In this approach, the power management considers combination of topology control, energy aware routing as well as sleep management, thus combining the information and functions from different networking layers to achieve effective power management. The problem of achieving minimum power configuration of the sensor network considering these factors is addressed, and minimum power configuration protocol (MPCP) is proposed.

Developing hierarchical clusters in the network is another approach employed for power conservation in sensor networks. In these hierarchical clusters, the cluster members communicate only with their immediate cluster head and the cluster head aggregates the data and sends them to the cluster head of the upper level. Thus, the cluster members need only to communicate with its local cluster head and can use low transmission power for communication. In addition, as data aggregation is performed at each level, the amount of data transmitted is reduced without loss of obtained information. This reduction in data requirement also supports power conservation. A distributed and randomized clustering algorithm, which generates a hierarchical cluster architecture, is proposed by Bandyopadhyay and Coyle [80]. In their approach, the cluster architecture is developed in bottom up manner and at each level the participating sensor nodes volunteer to become cluster heads with a given probability. Data aggregation is performed at the cluster head at each level for improved power conservation. Moreover, an energy efficient clustering scheme (EECS) is proposed by Ye et al. [81]. Remaining energy based cluster head election mechanism using broadcast 
messages, is proposed for the cluster head selection and request response based cluster formation is employed for cluster member selection, in this scheme.

Heinzelman et al. [55][82] have proposed low-energy adaptive clustering hierarchy (LEACH), a protocol architecture for sensor networks that support energy efficiency through cluster based routing and data aggregation. The clusters are dynamically formed in a distributed manner by each node electing itself as a cluster head with a certain probability, and then announcing itself as a head. The other nodes select the head that is closest to them as their cluster head and joins that cluster. The cluster head is responsible for generating a schedule for the cluster members to send data to cluster head. In addition, the cluster head performs the data aggregation to eliminate the redundancies. Moreover, the cluster heads are rotated and clusters are regenerated periodically to achieve even energy consumption across the network, which results in extending the sensor network lifetime.

Shah and Rabaey [83] proposed an energy aware routing algorithm designed to provide extended network lifetime for sensor networks. In this algorithm, the routes are dynamically generated as required and the route selection is based on energy left in the nodes along the path, as well as the energy consumption of the path. This combination provides energy-efficiency through the minimum energy path selection approach and the balancing of energy consumption at nodes by considering the energy left in the node. Schurgers and Srivastava [84] proposed a combination of data aggregation and uniform energy utilization at sensor nodes to achieve extended network lifetime. In this routing algorithm, the uniform energy utilization is achieved by selecting paths in a manner so that the data flows will select different paths. A random path selection approach, energy 
left in the nodes based path selection approach and a stream based approach are proposed for this purpose. All these methods perform node selection from local information. In addition, data aggregation is performed at nodes where more than one data flow is present to achieve power conservation. Directional Source-Aware Protocol (DSAP) is proposed by Salhieh et al. [85]. This protocol assumes fixed network topology and availability of global topology information. The routing is performed using a unique identifier assigned to each node. This identifier is calculated by assigning a 2D or 3D mesh topology to the sensor network. Energy efficiency is attained through the elimination of route generation and power aware path selection.

\subsubsection{Energy Efficiency in MAC Layer}

Sensor MAC (S-MAC), energy-efficient and self-configuring MAC protocol for wireless sensor networks is proposed by Ye et al. [86][87]. S-MAC too uses periodic sleep scheduling with low duty cycle for effective power management. Each sensor node decides its sleep schedule independently and the neighboring nodes synchronize their sleep schedules through exchange of periodic synchronization messages. This synchronization reduces the control overhead of the protocol, which improves energy efficiency. In addition, the sensor nodes create virtual clusters based on the common sleep schedule to effectively support adaptive wakeup depending on the traffic demands

of the network. Moreover, S-MAC uses in-channel signaling and message passing to reduce overhearing unnecessary traffic and collisions, which will also support power conservation.

$\mathrm{Lu}$ et al. [88] proposed DMAC, an adaptive energy-efficient and low-latency MAC protocol for wireless sensor networks. Similar to S-MAC, DMAC employs 
synchronized sleep scheduling of nodes to achieve effective power conservation. The protocol is inspired by the observation that in sensor networks with a single sink, data delivery paths from sources to sink are in a tree structure. It is developed to deliver data along this tree structure. Thus, the sleep schedule of the nodes in the different levels of the tree are arranges in a staggered manner. In addition, the duty cycle is dynamically adjusted according to the traffic load, by passing the information about remaining data load along with the data packets. Moreover, a traffic prediction mechanism with channel reservation through a control packet is used to reduce channel contention and collisions.

Timeout MAC (T-MAC) proposed by Dam and Langendoen [89] also uses synchronized sleep scheduling with dynamic duty cycle adjustment for power conservation. In this protocol, the active part of the duty cycle is dynamically ended when no data transmission is present. Thus, when the traffic load is low the duty cycle will be small and in presence of high traffic, the nodes will have large duty cycles to support the traffic requirements. Similar to S-MAC [87], T-MAC employs synchronization and virtual clustering of nodes, through synchronization messages. However, in this case, upon receiving the synchronization message the receiving node will adopt the combined schedule of both the nodes and notify the sender with its new schedule.

Polastre et al. [90] proposed Berkeley MAC (B-MAC), another energy efficient MAC protocol with sleep scheduling. It combines low power operation, effective collision avoidance, and high channel utilization, for efficient power conservation. In contrast to S-MAC [87], D-MAC [88] and T-MAC [89], B-MAC uses asynchronous sleep scheduling and preamble messages are used for waking up nodes. An adaptive preamble sampling method is employed to reduce the duty cycle of sleep scheduling to 
achieve low power operation. Collision avoidance is achieved through clear channel assessment and packet backoff.

Liu et al. [91] proposed convergent MAC (CMAC), an energy efficient MAC protocol and a geographical data forwarding algorithm for wireless sensor networks. CMAC too uses low duty cycle asynchronous sleep scheduling to achieve energy efficiency. It incorporates aggressive RTS (ready to send) messages with double channel check and convergent packet forwarding to achieve this. In this protocol, when a node wants to send some data, it anycasts a burst of RTS messages with short fixed gaps. Upon receiving this packet, nodes that are eligible to forward this packet will reply with CTS (clear to send) message and then the data is sent to the selected forwarder. In order to allow low duty cycles, channel sensing mechanism called double channel check is employed. In this approach, rather than continuously sensing the channel, the channel is sensed twice with a short gap. In addition, to reduce the overhead of using the anycast based forwarding, after the initial path setup, the route is converged to a unicast based routing. For this purpose, if the forwarder selected by the anycast forwarding has a near-optimal routing metric, then unicast based forwarding is employed for the remaining data forwarding.

Energy and rate based MAC protocol for wireless sensor networks is proposed by Kannan et al. [92]. This algorithm is based on S-MAC [87] and uses sleep scheduling to achieve power conservation. However, in contrast to above algorithms, it adjusts the duty cycle based on the criticality of the sensor node, and the nodes sleep only during the time slots allocated to them. The criticality of a sensor node is assessed based on the energy left in the sensor node as well as the amount of traffic flows going through the sensor 
node. An energy-efficient MAC protocol and a geographic random data forwarding mechanism, (GeRaF) are proposed by Zorzi and Rao [93]. The protocol uses periodic sleep scheduling to achieve effective power management. The geographic random packet forwarding method randomly selects the best node to forward the packet based on the geographic information.

Wan et al. [16][17] proposed an energy-efficient and low latency MAC protocol with an energy-aware anycast based data forwarding mechanism for wireless sensor networks. The MAC protocol employs an opportunistic sleep scheme to adaptively balance the energy consumption and end-to-end delay of the network. The energy aware anycast based data forwarding mechanism employs geographic data forwarding with forwarding node selection based on geographic optimality as well as on the energy left in the sensor nodes. We incorporate this protocol to our self-organization algorithm to achieve effective power management. A detailed discussion of the protocol and achieving of effective power management using the protocol is provided in Chapter IV.

\subsection{Summary}

In this chapter, first we briefly introduce the acoustic source localization strategies proposed in literature. Among the strategies discussed, the TDOA information based localization strategies provide a good tradeoff between the localization accuracy and the computational demands. In addition, the TDOA based strategies can be easily extended to track multiple targets.

The second part of the chapter provides a detailed discussion on energy-efficient target tracking using wireless sensor networks with primary focus on acoustic target tracking and the energy efficient networking protocols for wireless sensor networks. It is 
evident from this discussion that, for target tracking applications, simple localization and tracking methods are preferred compared to robust, resource demanding methods. Moreover, it is also apparent that incorporating sleep-wakeup management schemes is the most effective and popular power conservation methodology used in wireless sensor networks. In addition to sleep-wakeup management mechanisms, utilizing less resource demanding processing methods, reducing the communication complexity of the methods, localized processing and decision making, and data aggregation, are popular means used to achieve energy-efficiency in wireless sensor networks for target tracking applications. Furthermore, it is evident from the discussion that cross layer power management strategies have higher capability to effectively support efficient power management in wireless sensor networks compared to the isolated power management strategies employed at the different network layers. 


\section{CHAPTER III}

\section{TARGET LOCALIZATION AND TRACKING}

This chapter provides the details of the methods used for target localization and tracking. First we present the target localization method used in our algorithm. The second section analyzes the major error sources of the localization method. This forms the foundation for the node selection method used in the proposed self-organization algorithm. The details of the Kalman filter employed by the self-organization algorithm for target state estimation and prediction are discussed in the last section.

\subsection{Target Localization}

Time difference of arrival (TDOA) based localization strategy provides a good trade-off between localization accuracy and simplicity [6][20]. Thus, TDOA based acoustic localization is employed in our work. In TDOA based localization, the relative time delay of signal arrivals at two acoustic sensors are estimated from the received acoustic signals. Then, the locus of the target is estimated from the obtained time delay. The intersection of multiple such loci is used to obtain the estimation of the target location $[6][7]$.

\subsubsection{TDOA Estimation}

Several methods [94-96] are proposed for the correlation based estimation of TDOA of two acoustic signals received at acoustic sensors which are located close to each other with sufficient separation. In these methods, the cross-correlation function of the two signals is calculated and filtered in some optimal sense, and the maximum is obtained using a peak detector [4][7][20][94-99]. The time difference value, which corresponds to the obtained maximum, is the TDOA estimation. 


\subsubsection{Target Localization}

Once the TDOA estimation is obtained, the locus of the target can be generated from the location information of the acoustic sensors and the estimated TDOA. Brandstein et al. [6][26] analyzed the geometric relationship between the sensors and the target. For a given sensor pair location and a TDOA value, the locus of the target is a hyperboloid centered at the sensor pair midpoint. The sensor pair baseline (line connecting two sensors) is the axis of symmetry. The curvature of the hyperboloid is determined by the TDOA value and the sensor pair baseline distance. However, under the far field assumption this hyperboloid can be approximated by a circular cone with its center at the sensor pair midpoint [6]. Sensor pair baseline is the axis of the cone. The TDOA value and the sensor pair baseline distance determine the aperture of the cone. The far field assumption states that the distance between the two sensors should be very small compared to that of the sensors and the target [6]. The target location can be calculated from three or more such loci.

In our work, as we restrict the scope to tracking ground targets and model the sensor network to be deployed on the ground, the localization becomes 2D localization problem. For the $2 \mathrm{D}$ scenario, the locus is a parabola and the cone approximation becomes a pair of straight lines starting from sensor pair midpoint. This approximated locus can be represented by the sensor pair baseline and the bearing of locus with respect to the sensor pair baseline. The locus of the target is shown in Figure 3.1. 


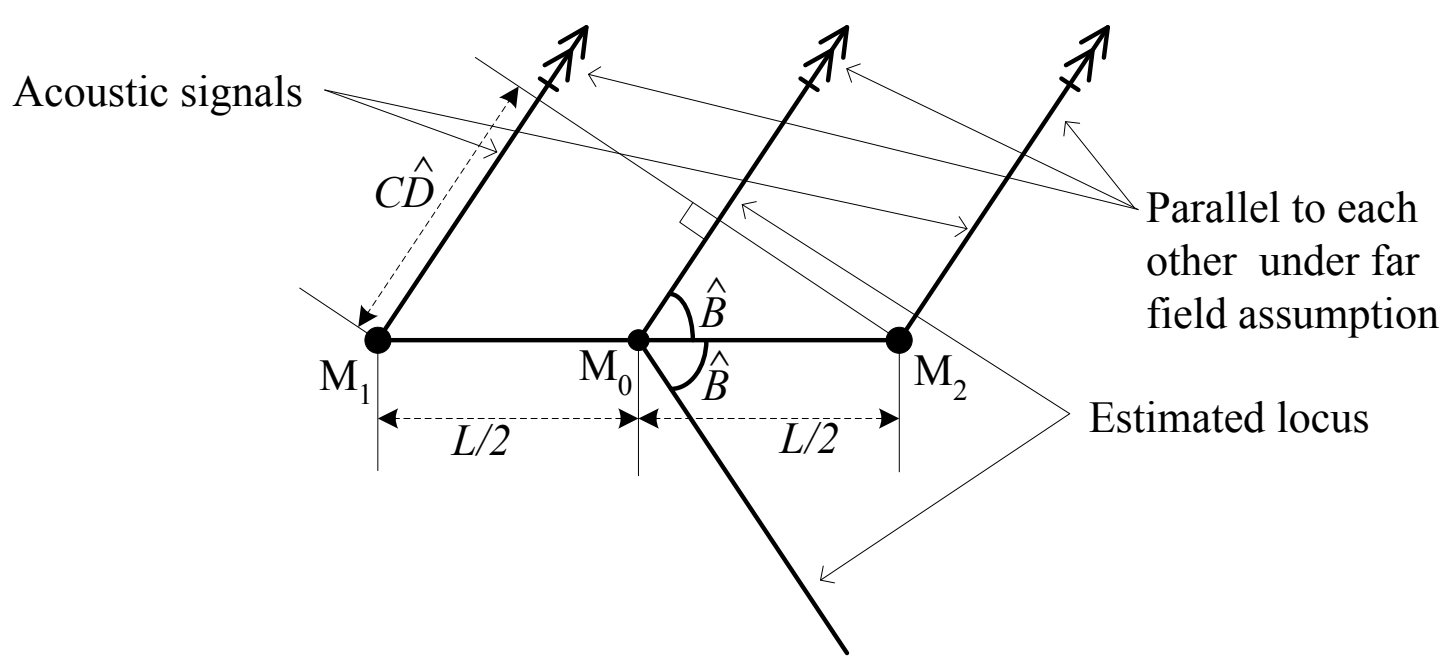

Figure 3.1 Approximated Locus of the Target with Sensor Pair Baseline

Here, $M_{1}$ and $M_{2}$ are positions of the two sensors. $M_{0}$ is the midpoint of $M_{1}$ and

$\mathrm{M}_{2} . \hat{B}$ is the estimated bearing of the target related to the sensor pair baseline. $\hat{D}$ is the estimated TDOA value and $C$ is the speed of sound. $L$ is the distance between the two sensors, termed sensor pair baseline distance. The bearing of the locus can be estimated by;

$$
\hat{B} \approx \cos ^{-1}\left(\frac{C \hat{D}}{L}\right)
$$

In the case of 2D localization scenario, two loci are enough. The target triangulation using two such loci is shown in Figure 3.2. 


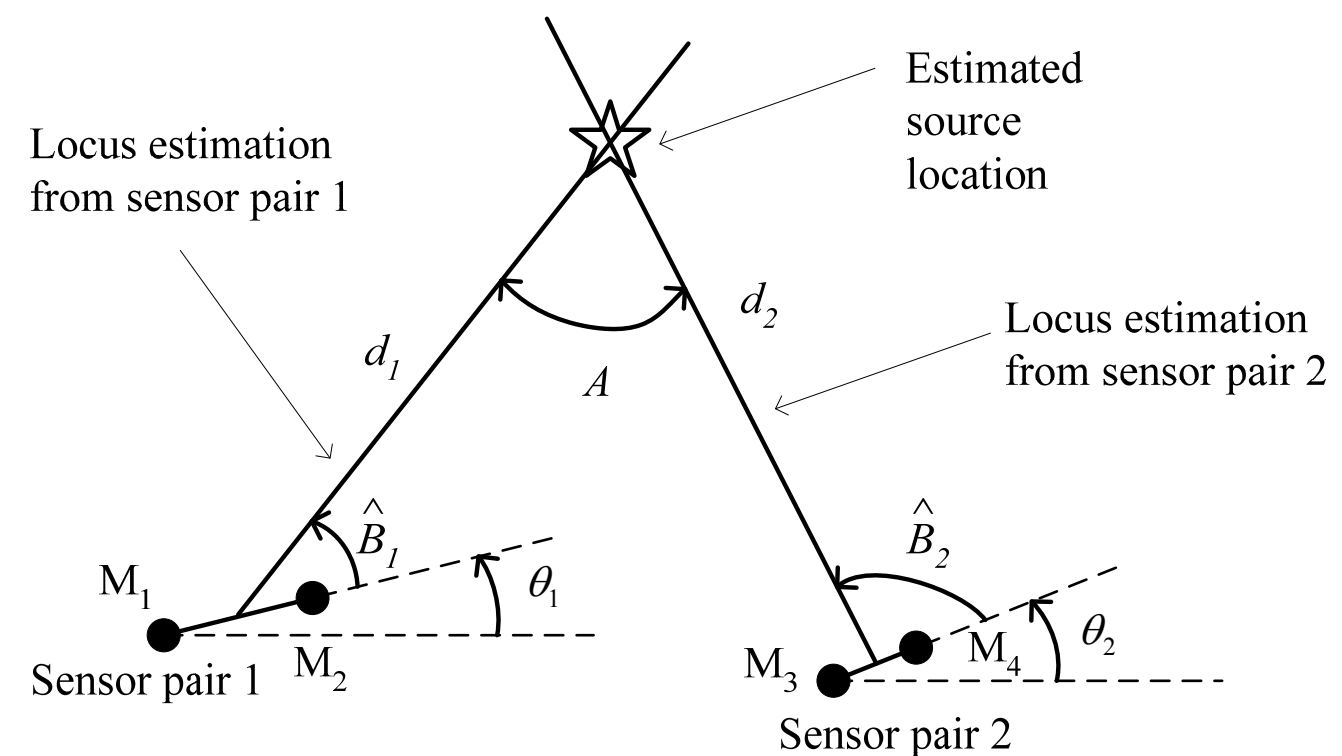

Figure 3.2 Target Triangulation Using Two Loci

Here, $\mathrm{M}_{1}, \mathrm{M}_{2}, \mathrm{M}_{3}$ and $\mathrm{M}_{4}$ are positions of the acoustic sensors. $\hat{B}_{1}$ and $\hat{B}_{2}$ are the bearing estimations from sensor pairs one and two, respectively. Distance from estimated target location to the sensor pair middle points are represented as $d_{1}$ and $d_{2}$. $A$ is the angle between two bearing lines, termed bearing angle. $\theta_{1}$ and $\theta_{2}$ are the angle between microphone pair baselines and the $x$ axis of the coordinate system. The source location, $(\hat{x}, \hat{y})$ can be estimated as;

$$
\begin{gathered}
\hat{x}=\frac{y_{2}-y_{1}+\tan \left(\hat{B}_{1}+\theta_{1}\right) x_{1}-\tan \left(\hat{B}_{2}+\theta_{2}\right) x_{2}}{\tan \left(\hat{B}_{1}+\theta_{1}\right)-\tan \left(\hat{B}_{2}+\theta_{2}\right)} \\
\hat{y}=\tan \left(\hat{B}_{1}+\theta_{1}\right)\left(\hat{x}-x_{1}\right)+y_{1}
\end{gathered}
$$

where $\left(x_{1}, y_{1}\right)$ and $\left(x_{2}, y_{2}\right)$ are the sensor pair midpoint for the first and the second sensor pair, respectively. Although use of more loci may improve the performance of the 
localization, it would increase the complexity of the node selection process. Therefore, we propose using only two loci for target triangulation.

\subsection{Localization Error Source Analysis}

The proposed self-organization algorithm has to select a set of sensors for the target localization using the localization method discussed in the previous section. In order to achieve accurate localization, the selection should be performed in a manner that minimizes the target localization error. In target tracking applications, the node selection is traditionally based on either to maximizing the information gain ([12][13][57]) or minimizing the target localization error $([9-11][100])$. The node selection methods employed in [12][13] and [57] perform node selection based on maximizing an information measure, which are defined to minimize the localization accuracy while minimizing the resource requirements.

Kadar [9] developed a geometry dilution of precision (GDOP) measure through the analysis of maximum likelihood estimation for the target localization problem. The GDOP measure relates the sensor bearing measurement errors to the target position estimation errors as a function of sensors-to-target geometry. A node selection method which selects the best three nodes that minimize the GDOP measure was developed in [100]. Torrieri [101] proposed a localization method using TDOA measurements and analyzed the bearing estimation error and the GDOP measure in details. Inspired by GDOP, Kaplan [10] developed the global node selection (GNS) method. GNS is a centralized method that performs node selection to minimize the expected filtered mean squared position error with the global knowledge of all node locations. Local node selection, a distributed version of the GNS was developed in [11]. All these node 
selection methods are designed for sensor networks with a sensor array at each node, where a single sensor node can provide the TDOA estimation or the DOA estimation. However, we consider a sensor network with only a single acoustic sensor at each sensor node and therefore these results cannot be directly applied for the node selection. Therefore, inspired by above work, we developed a node selection mechanism based on minimizing the localization error source, which considers sensor-to-source geometry. In order to form the basis for node selection, the major error sources of the localization method and propagation of error thought the localization process are analyzed here.

\subsubsection{TDOA Estimation Error}

Error for TDOA estimation using correlation strategies is studied in detail by Carter [94], Weiss et al. [97][102] and Ash \& Moses [4]. According to these results, the TDOA estimation error can be statistically bounded using Cramer-Rao lower bound (CRLB) under the constraint that the joint signal-to-noise ratio (SNR) is higher than a threshold value $\left(\mathrm{SNR}_{\mathrm{TH}}\right)$. This is the well known threshold effect in TDOA estimation [97]. In addition, CRLB only provides a lower bound of the root mean square (RMS)

error variance $\left(\sigma_{D}^{2}\right)$ for the TDOA estimation. According to Ash \& Moses [4], the TDOA estimation error variance is bounded by;

$$
\sigma_{D}^{2} \geq \frac{1}{8 \pi^{2} b T F_{c}^{2} S N R}, S N R>S N R_{T H}
$$

where $b$ is the signal bandwidth in $\mathrm{Hz}, T$ is the signal duration in seconds, and $F_{c}$ is the center frequency of the signal. $S N R$ is the joint signal to noise ratio and the $S N R_{T H}$ is the threshold value of the $S N R$. SNR is defined in Equation 3.5 [4][97]. 


$$
S N R=\frac{S N R_{1} S N R_{2}}{1+S N R_{1}+S N R_{2}}
$$

where $S N R_{1}$ and $S N R_{2}$ are the effective signal to noise ratios of the acoustic signals received at acoustic sensor one and two, respectively. The threshold value, $S N R_{T H}$ can be determined using the following equation [4][97].

$$
S N R_{T H}=\frac{6}{\pi^{2} b T}\left(\frac{F_{c}}{b}\right)^{2}\left[\phi^{-1}\left(\frac{b^{2}}{24 F_{c}^{2}}\right)\right]^{2}
$$

where,

$$
\phi(y)=\frac{1}{\sqrt{2 \pi}} \int_{y}^{\infty} e^{\frac{-t^{2}}{2}} d t
$$

When SNR drops below the threshold, the TDOA estimation error dramatically increases [4][94][97][103].

In passive acoustic source localization, we do not have control over most parameters in Equation 3.6. In addition, as the tracking is performed in real-time, the signal duration $(T)$ is limited. However, Ash \& Moses [4] have observed that under the spherical expansion and constant background noise assumption, the $S N R$ and the sensor separation distance are interrelated. Specifically the $S N R$ (defined in Equation 3.5) decreases $6 \mathrm{~dB}$ per doubling of the separation between the sensors [4]. Thus, by keeping the sensor separation distance below a threshold value $\left(L_{T H}\right)$, the $S N R$ can be kept above the required threshold value and a dramatic increase of the TDOA estimation error can be avoided. This maximum microphone separation distance $\left(L_{T H}\right)$, can be determined through off-line analysis and simulations. 


\subsubsection{Bearing Estimation Error}

Exact formulation of bearing estimation error would require statistics of the TDOA estimation. However, under the assumption that the probability density function of TDOA is concentrated near its mean value, standard deviation of the bearing estimation error can be approximated as a function of TDOA estimation error standard deviation [6][96] and is given by;

$$
\sigma_{B} \approx \frac{C \sigma_{D}}{L \sin (B)}
$$

where, $\sigma_{D}$ is the TDOA estimation error standard deviation, $L$ is the sensor pair baseline distance, and $B$ is the estimated bearing of the target related to the sensor pair baseline. The term " $L \sin (B)$ " reflects the projected length of the sensor pair base line on to an axis that is perpendicular to the line connecting the target and the sensor pair midpoint, and is termed the effective length of the sensor pair baseline. It is evident from Equation 3.8 that a large effective baseline length will result in smaller bearing estimation error.

\subsubsection{Triangulation Error}

Position estimation uncertainty in target triangulation using two microphone pairs is shown in Figure 3.3. Here, $\mathrm{M}_{1}, \mathrm{M}_{2}, \mathrm{M}_{3}$ and $\mathrm{M}_{4}$ are positions of the acoustic sensors. $B_{1}$ and $B_{2}$ are the actual bearings for actual target location from midpoints of sensor pairs one and two, respectively. Distance from actual target location to the sensor pair midpoints are represented as $\mathrm{d}_{1}$ and $\mathrm{d}_{2}$. $A$ is the actual bearing angle. $\theta_{1}$ and $\theta_{2}$ are the angle between microphone pair baselines and the $x$ axis. $\sigma_{B 1}$ and $\sigma_{B 1}$ represent the bearing estimation error standard deviations for sensor pairs one and two. The area of 
positional uncertainty (shaded area in Figure 3.3) is considered as the variance of position estimation error and can be approximated by;

$$
\sigma_{x} \sigma_{y} \approx \frac{4 d_{1} d_{2} \sigma_{B 1} \sigma_{B 2}}{\sin (A)}
$$

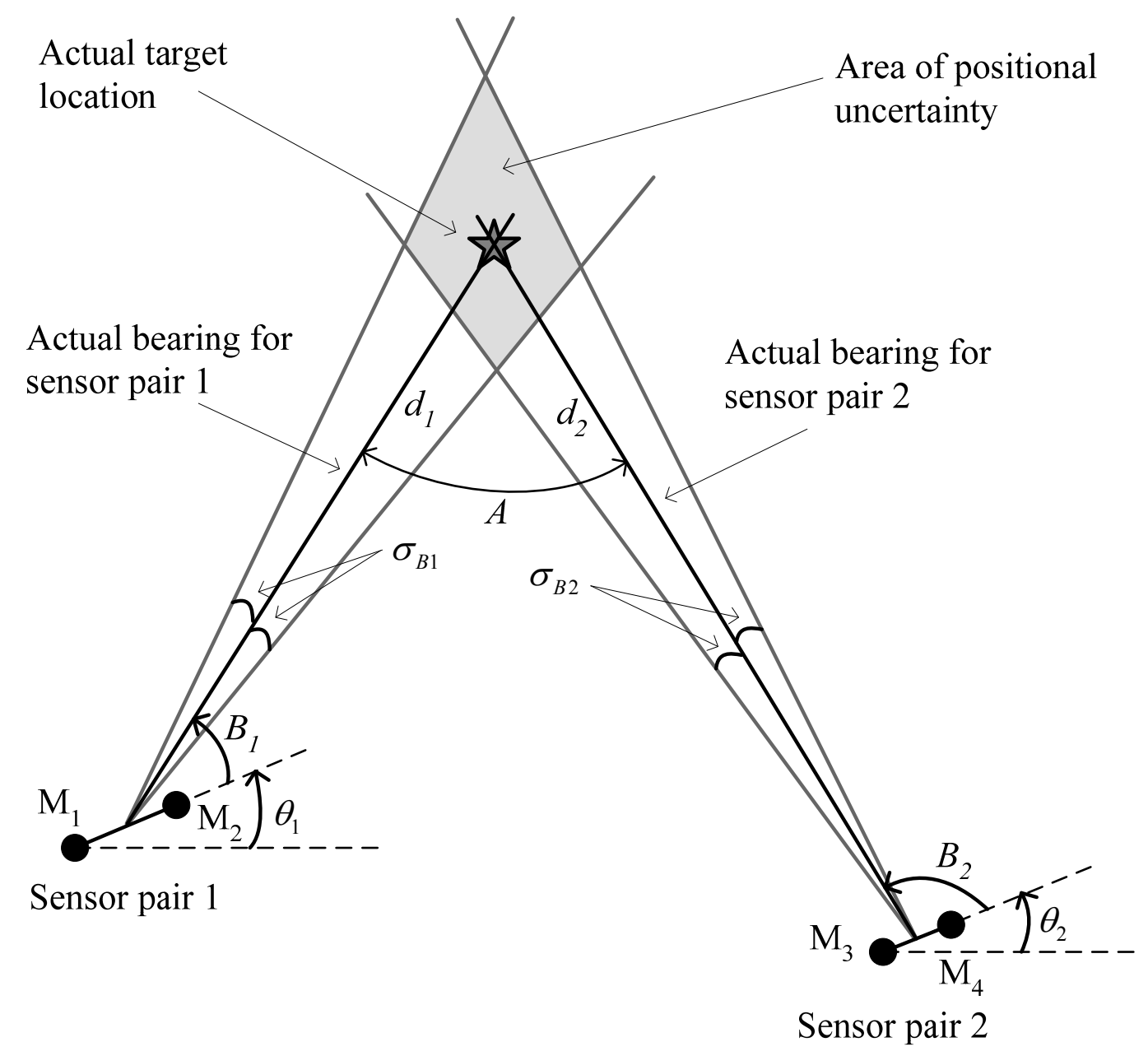

Figure 3.3 Position Estimation Uncertainty in Target Triangulation

Equation 3.9 formulates the relationship between the target position estimation error and the bearing estimation error depending on the sensor-to-source geometry. According to this relationship, small position estimation errors can be achieved by selecting sensor nodes close to the target. In addition, the position estimation error can be minimized by 
selecting the sensor pairs that have the angle between the bearing lines $(A)$ close to $90^{\circ}$. Resembling conclusions have been drawn in [9] and [10].

Above conclusions are illustrated by the following simple simulation results. In this simulation, the localization method described in Section 3.1 was employed to localize an acoustic source located at the origin of the $x-y$ plane. In the first part of the simulation $d_{1}$ and $d_{2}$ were set to $100 \mathrm{~m}$. The first sensor pair was fixed in the field with the sensor pair midpoint at $(-100,0)$. The midpoint location of the second sensor pair was varied to achieve bearing angles $(A)$ of $\frac{\pi}{6}, \frac{\pi}{3}, \frac{\pi}{2}$ and $\frac{5 \pi}{6}$. The bearing estimations were generated by adding random noise to the actual bearing, where $\sigma_{B 1}$ and $\sigma_{B 2}$ were set to $1.25^{\circ}$. In the second part of the simulation, the bearing angle was set to $\frac{\pi}{3}$ and $\frac{\pi}{2}$. The distances $d_{1}$ and $d_{2}$ were set to $100 \mathrm{~m}$ in one scenario and $200 \mathrm{~m}$ in another. Localization of the target was simulated for $10^{6}$ times for each setting and the estimated target locations were observed. The results are shown in Figure 3.4 and Figure 3.5. In addition, the mean and the standard deviation of the position estimation error are tabulated in Table 3.1 and Table 3.2. The results clearly rationalize the above conclusions. 


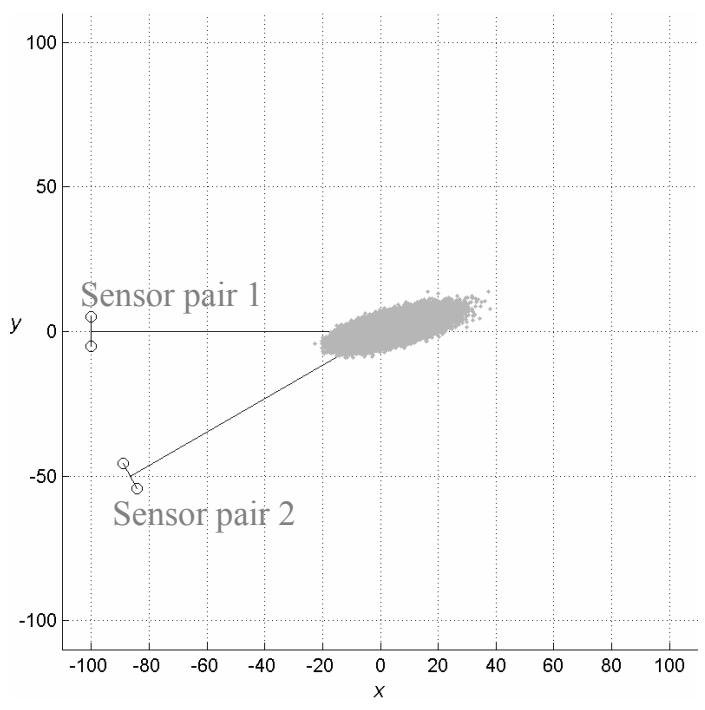

(a) $A=\frac{\pi}{6}$

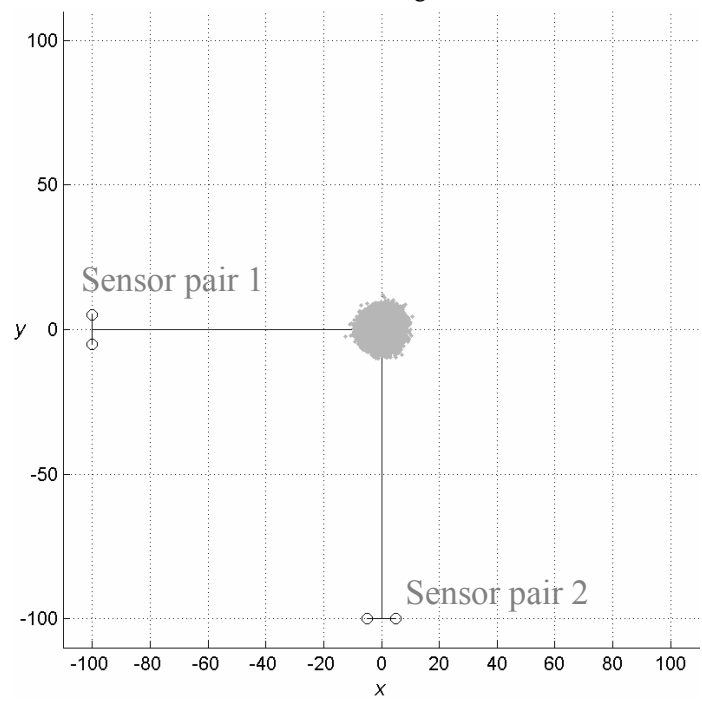

(c) $A=\frac{\pi}{2}$

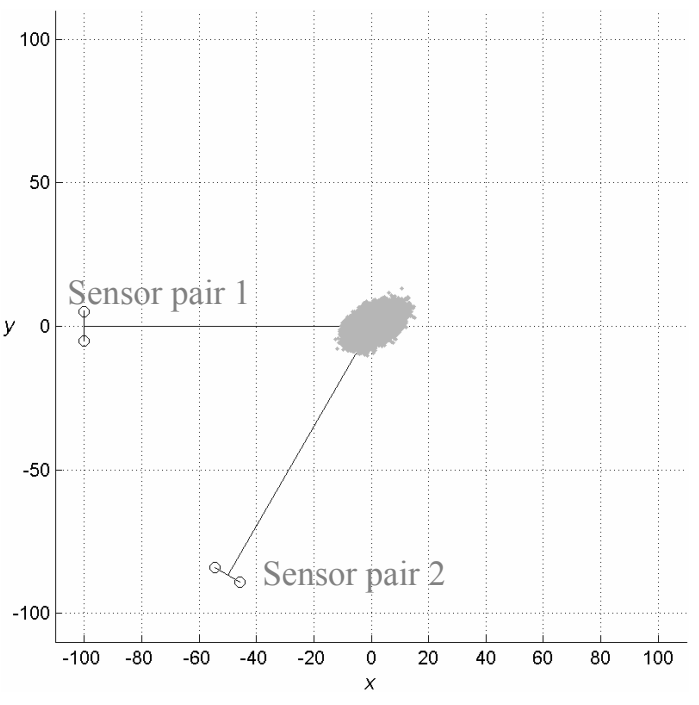

(b) $A=\frac{\pi}{3}$

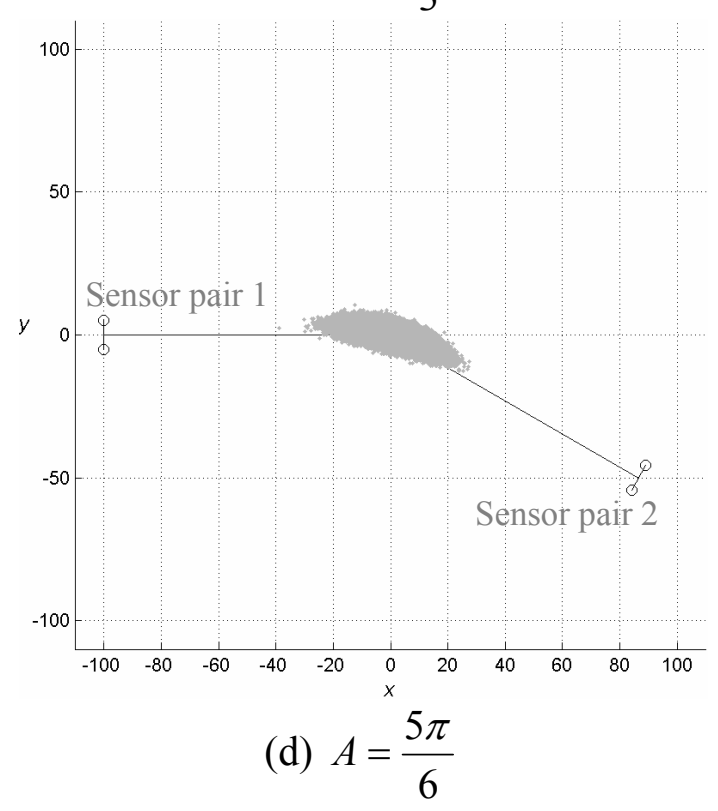

Figure 3.4 Effect of Bearing Angle on Triangulation Error

Table 3.1 Position Estimation Error Statistics for Different Bearing Angles

\begin{tabular}{|c|c|c|c|c|}
\hline Bearing Angle & $A=\frac{\pi}{6}$ & $A=\frac{\pi}{3}$ & $A=\frac{\pi}{2}$ & $A=\frac{5 \pi}{6}$ \\
\hline Mean Error & 5.1771 & 3.1102 & 2.7358 & 5.1523 \\
\hline $\begin{array}{c}\text { Standard } \\
\text { Deviation of } \\
\text { Error }\end{array}$ & 3.5331 & 1.7613 & 1.4320 & 3.4399 \\
\hline
\end{tabular}




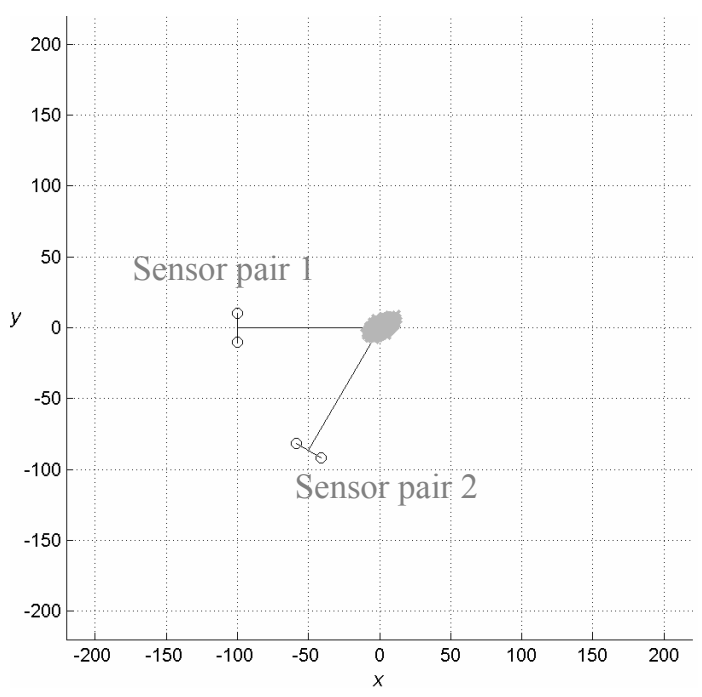

(a) $d_{1}=d_{2}=100$ and $A=\frac{\pi}{3}$

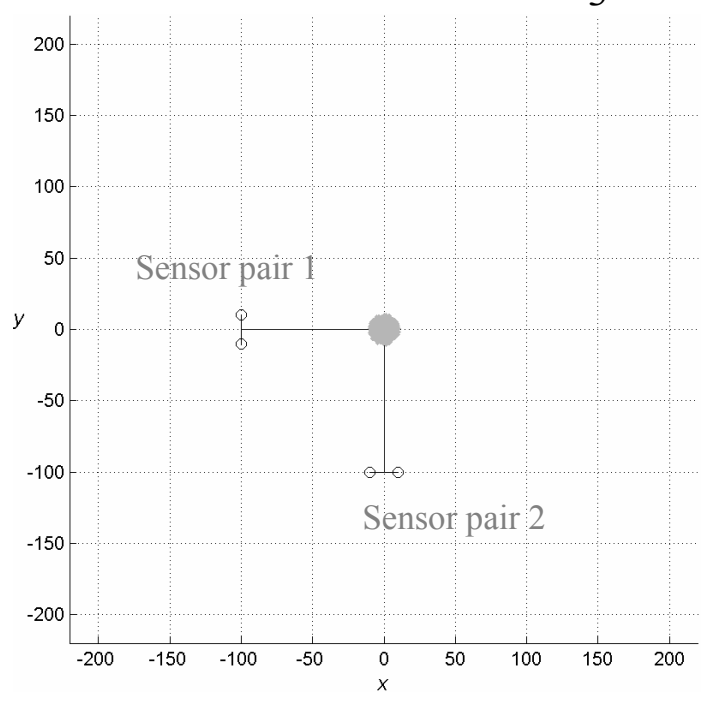

(c) $d_{1}=d_{2}=100$ and $A=\frac{\pi}{2}$

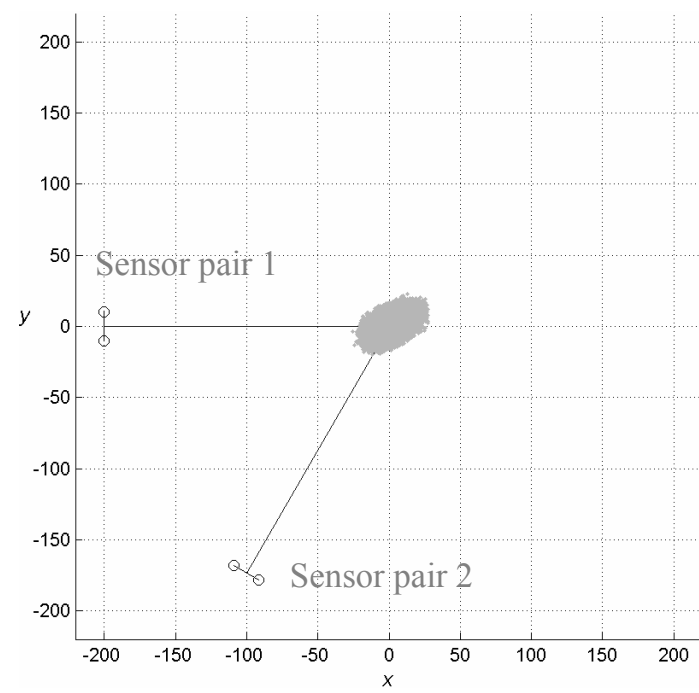

(b) $d_{1}=d_{2}=200$ and $A=\frac{\pi}{3}$

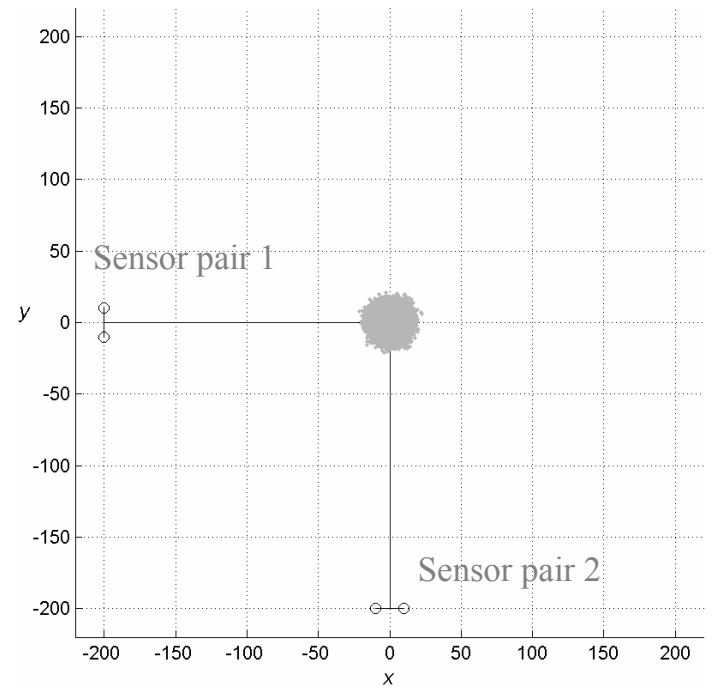

(d) $d_{1}=d_{2}=200$ and $A=\frac{\pi}{2}$

Figure 3.5 Effect of Distance from Sensor Pair Midpoint to Target on Triangulation Error

Table 3.2 Position Estimation Error Statistics for Different Distances from Sensor Pair Midpoint to Target

\begin{tabular}{|c|c|c|c|c|}
\hline Scenario & $d_{1}=d_{2}=100$ & $d_{1}=d_{2}=200$ & $d_{1}=d_{2}=100$ & $d_{1}=d_{2}=200$ \\
\hline Mean Error & 3.1103 & $A=\frac{\pi}{3}$ & $A=\frac{\pi}{2}$ & $A=\frac{\pi}{2}$ \\
\hline $\begin{array}{c}\text { Standard } \\
\text { Deviation of } \\
\text { Error }\end{array}$ & 1.7609 & 3.5146 & 1.4336 & 2.7365 \\
\hline
\end{tabular}




\subsection{Target Tracking}

In target tracking applications, different approaches have been employed for measurement improving, target state estimation and prediction. Kalman filter, Extended Kalman filter (EKF), Un-scented Kalman Filter (UKF), Particle filter and their variations are some of the popular methods used for target tracking applications $[10][11][27][30][31][41][104][105]$. We employ a Kalman filter based tracking method for target position and speed estimation and position prediction. The details of the Kalman filter employed are discussed in this section.

The measurement to the Kalman filter is the target location estimates using the above discussed localization method. A constant velocity dynamic model with a constant sampling interval $(\tau)$ is used and the acceleration is treated as process noise in the derivation. Position and speed of the target were selected to represent the target state. Let

the state of the system be $\boldsymbol{x}(k)=[x, y, \dot{x}, \dot{y}]^{\mathrm{T}}$. Then, the system is described using the following system and measurement equations.

$$
\begin{aligned}
& \boldsymbol{x}(k+1)=\boldsymbol{F}(k+1, k) \boldsymbol{x}(k)+\boldsymbol{G}(k+1, k) \boldsymbol{w}(k) \\
& \boldsymbol{z}(k+1)=\boldsymbol{H}(k+1) \boldsymbol{x}(k+1)+\boldsymbol{v}(k+1)
\end{aligned}
$$

where

$$
\boldsymbol{F}(k+1, k)=\left[\begin{array}{cccc}
1 & 0 & \tau & 0 \\
0 & 1 & 0 & \tau \\
0 & 0 & 1 & 0 \\
0 & 0 & 0 & 1
\end{array}\right]
$$




$$
\begin{gathered}
\boldsymbol{G}(k+1, k)=\left[\begin{array}{cc}
0.5 \tau^{2} & 0 \\
0 & 0.5 \tau^{2} \\
\tau & 0 \\
0 & \tau
\end{array}\right] \\
\boldsymbol{H}(k+1)=\left[\begin{array}{llll}
1 & 0 & 0 & 0 \\
0 & 1 & 0 & 0
\end{array}\right]
\end{gathered}
$$

$\boldsymbol{w}(k)$ and $\boldsymbol{v}(k)$ are zero-mean white Gaussian process noise and measurement noise with covariance $\boldsymbol{Q}(k)$ and $\boldsymbol{R}(k)$, respectively. $\boldsymbol{Q}(k)$ and $\boldsymbol{R}(k)$ are given by;

$$
\begin{gathered}
\boldsymbol{Q}(k)=\left[\begin{array}{cc}
\sigma_{a x}^{2} & 0 \\
0 & \sigma_{a y}^{2}
\end{array}\right] \\
\boldsymbol{R}(k)=\left[\begin{array}{cc}
\sigma_{x}^{2} & 0 \\
0 & \sigma_{y}^{2}
\end{array}\right]
\end{gathered}
$$

$\sigma_{a x}^{2}, \sigma_{a y}^{2}$ are the $x$ and $y$ components of disturbance processes variance and $\sigma_{x}^{2}, \sigma_{y}^{2}$ are the variances of source localization errors in $x$ and $y$ directions. Then, with the knowledge of previous state estimation $\hat{\boldsymbol{x}}(k \mid k)$, the estimation error covariance matrix for the previous state $\boldsymbol{P}(k \mid k)$, and the currant measurement, the currant state of the target $\hat{x}(k+1 \mid k+1)$ can be estimated by,

$$
\hat{\boldsymbol{\lambda}}(k+1 \mid k+1)=\boldsymbol{F}(k+1, k) \hat{\boldsymbol{\lambda}}(k \mid k)+\boldsymbol{K}(k+1)[z(k+1)-\boldsymbol{H}(k+1) \boldsymbol{F}(k+1, k) \hat{\boldsymbol{x}}(k \mid k)]
$$

The Kalman gain matrix, $\boldsymbol{K}(k+1)$ of the above equation, can be calculated using following equations.

$$
\begin{aligned}
& \boldsymbol{P}(k+1 \mid k)=\boldsymbol{F}(k+1, k) \boldsymbol{P}(k \mid k) \boldsymbol{F}^{\mathrm{T}}(k+1, k)+\boldsymbol{G}(k+1, k) \boldsymbol{Q}(k) \boldsymbol{G}^{\mathrm{T}}(k+1, k) \\
& \boldsymbol{S}(k+1)=\boldsymbol{H}(k+1) \boldsymbol{P}(k+1 \mid k) \boldsymbol{H}^{\mathrm{T}}(k+1)+\boldsymbol{R}(k+1)
\end{aligned}
$$




$$
\boldsymbol{K}(k+1)=\boldsymbol{P}(k+1 \mid k) \boldsymbol{H}^{\mathrm{T}}(k+1) \boldsymbol{S}^{-1}(k+1)
$$

The estimation error covariance matrix of the current state $\boldsymbol{P}(k+1 \mid k+1)$, is required for the next state estimation and can be calculated using;

$$
\boldsymbol{P}(k+1 \mid k+1)=[\boldsymbol{I}-\boldsymbol{K}(k+1) \boldsymbol{H}(k+1)] \boldsymbol{P}(k+1 \mid k)
$$

More complex system representation can easily be substituted into above system model to obtain more accurate results with the penalty of higher computational intensity. 


\section{CHAPTER IV}

\section{THE ENERGY-EFFICIENT AND DISTRIBUTED SELF-ORGANIZATION ALGORITHM}

The developed energy-efficient and distributed self-organization algorithm is presented in this chapter. First the overview of the overall design is provided. The proposed energy aware node selection criterion that minimizes the localization error is discussed in the second section. This is followed by the details of the energy-efficient self-organization algorithm and the distributed message passing protocol developed to implement the proposed self-organization algorithm. Finally the incorporation of the sleep-wakeup management scheme with the cross layer wakeup probability adjustment and the other cross layer enhancements incorporated to the self-organization algorithm are discussed.

\subsection{Design Overview}

Major focus of the self-organization algorithm is to select a sensor node group for target localization and tracking in a distributed manner while balancing the energy efficiency and the target tracking performance. An overview of the design of the energy-efficient and distributed self-organization algorithm is provided here.

\subsubsection{System Model}

The scope of the work is to track a moving ground target using a wireless acoustic sensor network. Each sensor node in the network consists of a single microphone for sensing, a microprocessor to provide information processing capabilities, a wireless interface for communication and a battery for power supply. In addition, the following assumptions are made about the sensor network. First, it is assumed that each node knows 
its location and all sensor nodes are time synchronized. Various sensor node self-localization and time synchronization methods have been proposed in the literature [4][109-119]. However, the self-localization and the time synchronization of the wireless sensor network are beyond the scope of our work. Second, it is assumed that sensor nodes can operate in two states, sleep state and active state. When the sensor node is in active state, the node is fully functional and ready to send/receive data packets. When it is in the sleep state, it will turn off its wireless radio interface and operate in the low power mode. However, a sensor node in the sleep state is still capable of capturing acoustic signals. In addition, it is assumed that each sensor node will have a rough estimation of the number of nodes that are in its one-hop neighborhood.

\subsubsection{Design Goals}

The algorithm design for wireless sensor networks is a challenging task due to the various limitations inherent to the sensor networks and the application domains. Resource limited and application specific nature of the requirements are two major constraints present in wireless sensor networks. Thus, the designing requires a careful consideration of many critical factors such as fault tolerance, scalability, hardware constraints, production cost, sensor network topology, environment and power consumption [1][13]. In particular, the proposed energy efficient distributed self-organization algorithm has been developed based on the following major design considerations.

\section{A. Power Consumption}

The sensor nodes are battery powered and thus have a limited energy supply. In addition, in most practical scenarios once they are deployed, the very limited accessibility of these sensor nodes makes battery replacement or recharging impossible. Thus, power 
limitation is a major constraint in sensor network algorithm design, which calls for an effective power management mechanism for such an acoustic sensor network to become practical [1-3][5][13][106][107]. For target tracking applications, our design goal of the algorithm is to achieve extended network lifetime while maintaining satisfactory target tracking performance.

\section{B. Scalability}

Depending upon the application, the size of the sensor network may vary from a few nodes to thousands (or even millions) of nodes. Often, sensor networks are densely deployed [1][2][5][13][106]. For the developed algorithms to work in these settings, scalability is a major design consideration. To make the developed algorithm scalable, it should be distributed in nature and only rely on local information exchange for self-organization. Moreover, the algorithm should be able to effectively exploit the high node density to prolong the lifetime of the sensor network by incorporating efficient power management.

\section{Resource Limitations}

The individual nodes in a sensor network usually have limited processing capabilities, memory and communication bandwidth due to the energy and cost considerations [1][13]. However, they are required to generate accurate results under varying environment conditions. Thus, it is essential that the processing techniques used in sensor network applications are capable of operating with limited resources, while providing good quality of service. For target tracking applications, careful selection of target localization and tracking techniques would enable achieving the tradeoff between the desired tracking performance and the resource demand in the sensor nodes. In 
addition, the communication requirements for the self-organization and target tracking should be minimized to support efficient operation under limited communication bandwidth.

\subsubsection{Overview of the Design}

The resource aware TDOA based localization method discussed in Chapter III is used for the target localization. To achieve localization using this method, two bearing estimations are required. However, as the sensor nodes contain only a single acoustic sensor, a single sensor node is not capable of generating a bearing estimation on its own. Thus, the self-organization algorithm has to select a group of sensor nodes to form a virtual acoustic sensor array for target localization. The selected sensor group is termed the localization sensor group (LSG). It consists of two sensor pairs, which independently generate their own bearing estimations. In each sensor pair, one node is selected to perform the bearing estimation and it is referred as the master sensor node. The other node is termed slave sensor node. In addition, one of the sensor pairs will be selected to perform the triangulation and target tracking, and that sensor pair is called the master sensor pair (MSP). The other sensor pair is termed slave sensor pair (SSP). Thus, the LSG consists of the master and the slave nodes of the master sensor pair, and the master and the slave nodes of the slave sensor pair. These four nodes are denoted as $M_{m}, M_{s}, S_{m}$, and $S_{m}$, respectively. The master and slave sensor pairs are denoted by $P_{m}\left(M_{m}, M_{s}\right), P_{s}$ $\left(\mathrm{S}_{\mathrm{m}}, \mathrm{S}_{\mathrm{s}}\right)$, and the localization sensor group is denoted by LSG $\left(\mathrm{P}_{\mathrm{m}}, \mathrm{P}_{\mathrm{s}}\right)$.

The node selection criterion for the formation of localization sensor group is developed based on the localization error source analysis presented in Section 3.2. In addition, the energy balancing is incorporated into the node selection process by 
including energy factors in the node selection criterion. The major goals of the proposed node selection criterion are to accomplish near-optimal localization performance and extended sensor network lifetime. A two-step master and slave node search process has been proposed to minimize the communication requirements and computational complexity of selecting the localization sensor group in a distributed manner. The Kalman filter based tracking method discussed in Section 3.3 is applied for the target state estimation and prediction. Moreover, the localization sensor group is dynamically updated to match the dynamics of the target.

In addition to energy aware node selection, a sleep-wakeup management scheme is incorporated into the algorithm for improved power management. For this purpose, an energy-efficient and latency sensitive MAC protocol with a location-based data forwarding mechanism proposed by Wan et al. [16][17] is integrated to the algorithm. This MAC protocol employs an opportunistic sleep management algorithm to improve energy conservation by reducing the energy loss from idle listening. To achieve efficient power management using this MAC protocol, a novel cross layer wakeup probability adjustment method is developed. This method combines event information from the application layer and network condition from the MAC layer to dynamically adjust the wakeup probability to achieve efficient power management while maintaining satisfactory target tracking performance. Moreover, a location based unicasting mechanism and a cross layer data packet priority assignment mechanism are introduced to the location based data forwarding mechanism, so that it can better support the self-organization algorithm. 


\subsection{Resource Aware Node Selection}

The core of the self-organization algorithm is the selection of proper localization sensor group based on the sensor source geometry to achieve near-optimal target localization performance. In addition, the maximization of the network life time should also be considered in the localization sensor group selection. Based on the localization error source analysis in Section 3.2, the localization sensor group should be selected to minimize the triangulation error variance defined in Equation 3.9. This can be achieved by performing the localization sensor group selection through a two-step selection process. The first step is the pairing of sensor nodes in such a way that the bearing estimation error (given by Equation 3.8) of the sensor pair is minimized. The second step is grouping the two best sensor pairs that will minimize the triangulation error, to form the localization sensor group.

\subsubsection{The Node Selection Criterion}

The bearing estimation accuracy depends on the TDOA estimation accuracy as well as the relative geometry of the target and the sensor pair. Thus, the cost functions that should be considered in the first step are TDOA estimation error variance (Equation 3.4) and the bearing estimation error variance (Equation 3.8). Specifically, the bearing estimation depends on the effective length of the baseline and the TDOA estimation error variance. According to bearing estimation error analysis (Section 3.2.2), the bearing estimation error can be minimized by maximizing the effective baseline length of the sensor pair. This requires the sensor pair baseline distance to be as large as possible. However, according to the TDOA estimation error analysis (Section 3.2.1), a longer sensor pair baseline distance will result in a lower $S N R$ (defined in Equation 3.5). The 
decrease in the $S N R$ will cause higher TDOA estimation error and if the $S N R$ drops below the required threshold $\left(S N R_{T H}\right.$ specified in Equation 3.6), the TDOA estimation error would dramatically increase. Trivially, higher TDOA estimation errors would cause higher bearing estimation errors. This analysis suggests contrasting requirements of sensor pair baseline distance for the bearing estimation error minimization. However, if the $S N R$ is maintained above the required threshold value $S N R_{T H}$, a dramatic increase in the TDOA estimation error can be avoided and in the region of $S N R \geq S N R_{T H}$, the increase in the TDOA estimation error due to the decrease of the SNR is comparatively small. According to the TDOA estimation error analysis, this can be achieved by maintaining the sensor pair baseline distance below the threshold distance $\left(L_{T H}\right)$. Thus, a good compromise can be achieved by maximizing the sensor pair baseline distance while maintaining it below the required threshold distance $\left(L_{T H}\right)$. In addition, the effective baseline length can be maximized by setting the sensor pair baseline in such a manner that it will face the target. This can be achieved by selecting the bearing to be close to $90^{\circ}$. Moreover, in the target localization process, the target locus approximates to a straight line under far field condition. Thus, in order to satisfy this constraint, the distance from the sensors to the target should be kept above a threshold value $\left(R_{F A R}\right)$. This threshold value $\left(R_{F A R}\right)$ will depend on $L_{T H}$ and can be determined off-line once the $L_{T H}$ is determined. Therefore, combining all these requirements, the sensor pairing should be performed to meet the following criteria.

1. The distance from each sensor to the estimated target location should be above the $R_{F A R}$. 
2. The sensor pair baseline distance should be maximized while maintaining it below the required threshold $L_{T H}$.

3. The bearing of the estimated target should be close to $90^{\circ}$.

Once the sensor pairing is completed, the second step performs the selection of two best sensor pairs for the localization sensor group. According to the triangulation error analysis (Section 3.2.3) and Equation 3.9, the sensor pairs that are best suited should have small bearing estimation errors, should be close to the target, and should form a bearing angle close to $90^{\circ}$ between them. In addition, to satisfy the far field condition, the distance from each sensor pair baseline midpoint to the estimated target location should be above the $R_{F A R}$. Thus, the selection of two sensor pairs should be performed based on the following criteria.

1. The distance from each sensor pair baseline midpoint to the estimated target location should be minimized, while maintaining it above the $R_{F A R}$.

2. The bearing estimation errors of the two selected sensor pairs should be minimal.

3. The bearing angle between the two sensor pairs should be close to $90^{\circ}$.

\subsubsection{Node Selection with Global Information}

The two-step node selection process can be carried out to match the criteria specified in the previous section with availability of global node information through two greedy searches. In the first search, the sensors that are located further than $R_{F A R}$ from the estimated target location are paired with the best node specified by the sensor pairing criterion. Specifically for each such node, the node that is located within $L_{T H}$ from its location and that minimizes the cost function given by Equation 3.8 is selected from set of nodes that are located further than $R_{F A R}$ from the estimated target location. This will 
generate the master-slave sensor pairs. The majority of the sensor pairs that are generated from this process will have the distance from sensor pair baseline midpoint to the estimated target location higher than $R_{F A R}$. However, it is possible that some sensor pairs may not match this criterion. Although these pairs do not match the criterion, the distance from sensor pair baseline midpoint to the estimated target location will be very close to $R_{F A R}$, under the condition that $L_{T H}$ is comparatively very small compared to $R_{F A R}$. Thus, this tiny error is negligible. In addition, the $R_{F A R}$ can be adjusted to a slightly higher value to compensate for this error.

The second search is carried out among the generated master-slave sensor pairs. In this search, the cost function specified by Equation 3.9, is evaluated for all the possible combinations of sensor pair groups, which consist of two sensor pairs. The sensor pair group that minimizes the cost function is selected as the localization sensor group. This process selects the optimal sensor group to match the above described sensor selection criteria.

\subsubsection{Resource Aware Node Selection Process}

The above discussed two-step process is highly computationally complex and requires global node information for both the searches. In addition, implementing this selection process in a distributed manner would require large amount of information exchange, making it highly demanding for limited communication resources. Therefore, this localization sensor group selection process will not have desired energy efficiency and scalability characteristics. However, the densely deployed nature of the wireless sensor network and the characteristics of the optimal localization sensor group can be effectively utilized to achieve a simplified localization sensor group selection process, 
which can perform the node selection based on geographically local information. This would result in a suboptimal, but highly desirable localization sensor group selection process.

First, to simplify the implementation and reduce the computational complexity and the communication demand of the localization sensor group selection process, the above discussed two steps are performed in reverse order. In this process, first the two master nodes are selected according to the above specified sensor pair selection criteria. For this selection, the bearing estimation error of the two sensor pairs and the sensor pair baseline midpoint information are required. However this information is not available as the master node selection is performed before master-slave sensor pair generation. Therefore, the following approximations are used for this process.

1. The bearing estimation error variances of all the sensor pairs are assumed to be the same, and to be equal to the bearing estimation error variance of an optimal sensor pair. Thus, when the bearing estimation error variance is not available, it is estimated by,

$$
C_{b e, \text { CONSTANT }}=\frac{C \sigma_{D, C O N S T A N T}}{L_{T H}}
$$

where $C$ is the speed of sound, $\sigma_{D, \text { CONSTANT }}$ is the preset constant TDOA estimation error variance and $L_{T H}$ is the predefined maximum allowable threshold distance for the sensor pair baseline.

2. In order to estimate the distance from sensor pair baseline midpoint to the target estimated location ( $d_{l}$ and $d_{2}$ in Equation 3.9), the sensor pair baseline midpoints 
are required. However, as this information is not available, the sensor pair baseline midpoints are approximated by the location of the master sensor nodes.

The rationale for the first approximation is that, as the sensor network is densely deployed, it will be possible for each sensor node to select a nearly optimal salve node for any target location. The second approximation is supported by the fact that under the condition of far field assumption, the sensor pair baseline is much smaller compared to the distance from target to the sensor nodes. Thus, the approximation of microphone pair baseline midpoint using the master sensor location will not introduce drastic errors. After these approximations, the cost function for master node selection $\left(C_{\text {geo }}\right)$ will be;

$$
C_{g e o}=\frac{\hat{d}_{1} \hat{d}_{2} C_{b e 1} C_{b e 2}}{\sin (\hat{A})}
$$

where $\hat{d}_{1}$ and $\hat{d}_{2}$ are the distance from the estimated target location to the master sensor of the sensor pair. $C_{b e 1}$ and $C_{b e 2}$ are bearing estimation error cost function values of two sensor pairs and when this information is not available, $C_{b e, C O N S T A N T}$ will be used as approximations for them. $\hat{A}$ is the bearing angle approximated from the lines connecting the two candidate master nodes and the estimated location of the target. The two nodes that minimize the cost function $C_{\text {geo }}$ (Equation 4.2) are selected as the master nodes.

The two optimal master sensor nodes should be very close to the estimated target location (see cost function $C_{g e o}$ in Equation 4.2) and have to be further than $R_{F A R}$ away from the estimated target location (to satisfy the far field condition). Thus, in densely deployed sensor networks they will be located just outside the boundary of the circular 
region, which is centered at the estimated target location and has a radius of $R_{F A R}$. The area that is outside this circular region is referred as the far field in the future discussion. The circular boundary that separates the circular region and the far field is referred as the far field boundary. With this observation, the scope of the master node selection process can be further limited to the nodes that are located in the far field and are very close to the far field boundary. This will enable the search to be carried out using only the information of nodes present in the region close to the far field boundary, eliminating requirement for global information exchange. Thus, this will reduce the communication demands of the search process. The ideas of far field, far field boundary and the high probability region of master node locations are shown in the following figure.

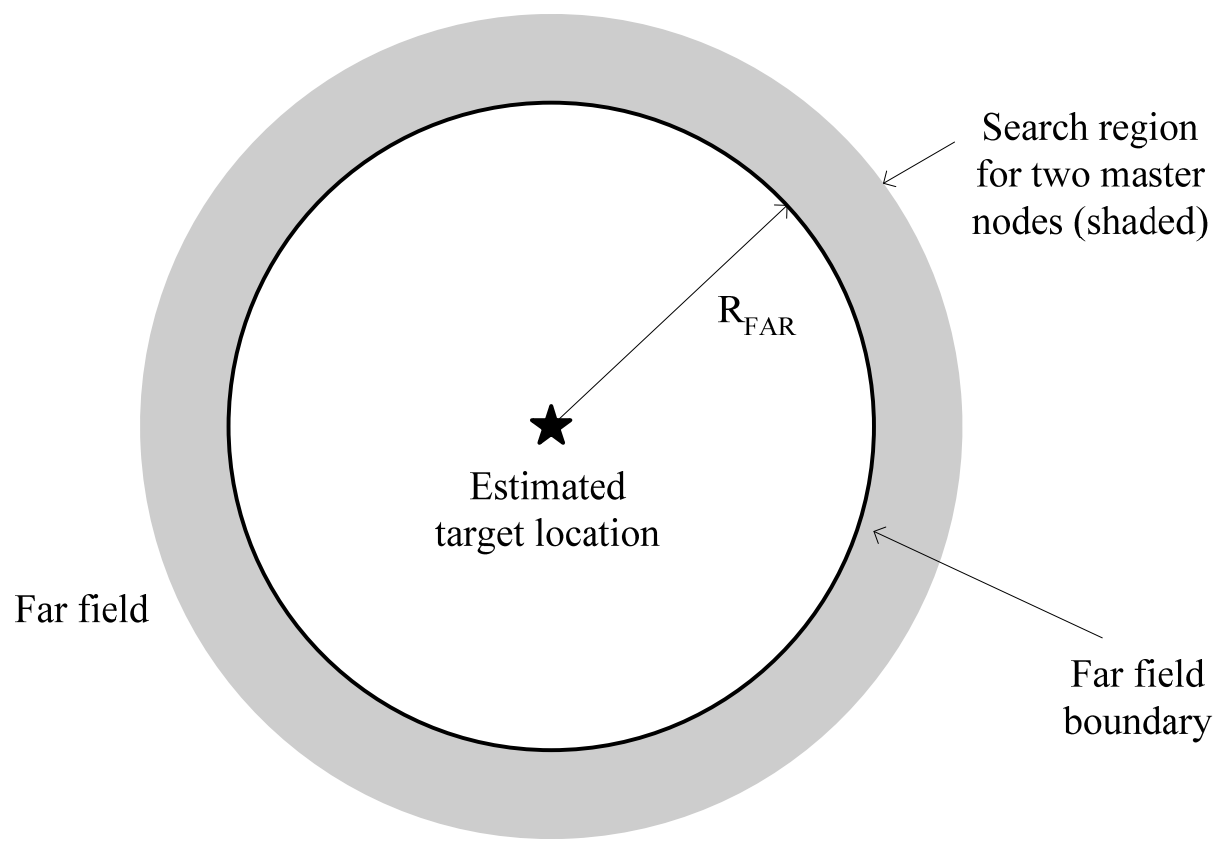

Figure 4.1 Master Node Search Area

Once the two master sensor nodes are selected, they will independently perform local searches for the respective slave nodes within their own one-hop neighborhoods to match the criterion specified in the sensor pairing process. In this process, the sensor 
nodes that are located within $L_{T H}$ from the master sensor node and in the far field are considered as candidate slave nodes. The cost function that will be used to evaluate the candidate slave nodes is given by,

$$
C_{b e}=\frac{C \sigma_{D, C O N S T A N T}}{L \sin (\hat{B})}
$$

where $C$ is the speed of sound, $\sigma_{D, C O N S T A N T}$ is the preset constant TDOA estimation error variance and $L$ is the sensor pair baseline distance. $\hat{B}$ is the bearing approximated for the estimated target location related to the sensor pair baseline. This cost function is derived from Equation 3.8 with the assumption the deviation in TDOA estimation error is negligible in the region of $S N R \geq S N R_{T H}$. The candidate slave node that minimizes the cost function $C_{b e}$ (Equation 4.3) is selected as the slave node. In addition, the sensor group that has the master node closer to the estimated target location will be selected as the master sensor pair. The two search processes are based on geographically local node information and have reduced computation and communication requirements. Thus this is more resource efficient and more desirable for distributed implementation.

\subsubsection{Incorporation of Energy Balancing into the Node Selection}

The resource aware node selection process described above does not consider the energy available in the nodes when selecting nodes. Therefore, in some scenarios, the same set of nodes may be selected for the tracking group, resulting in some of the nodes in the network being overused while some others are underused. This could result in unbalanced energy distribution in the sensor network causing a portion of network to run out of battery life quickly, weakening or even partitioning the wireless sensor network. 
Thus, energy consideration is incorporated into the node selection process in order to achieve better balanced energy utilization over the network. Moreover, in densely deployed sensor networks, there will be multiple localization sensor groups, that are very similar (in terms of tracking quality/objective function values) to the selected near-optimal localization sensor group. Thus, the selection of such a group which has higher energy left in its nodes will not cause a dramatic degradation in the target tracking performance. However, it will result in better network utilization and extended network lifetime.

The energy consideration is incorporated to the node selection process by introducing an energy element to the cost functions $C_{g e o}$ (Equation 4.2) and $C_{b e}$ (Equation 4.3). With the incorporation of the energy elements, the cost functions $C_{g e o}$ will be transformed into $C_{g e o, e}$ given by,

$$
C_{g e o, e}=\left(\alpha+(1-\alpha) \frac{E_{r, s n 1} E_{r, s n 2}}{2 E_{m}}\right) C_{g e o}
$$

where, $E_{r, s n 1}$ and $E_{r, s n 2}$ are the remaining energy of the two candidate master sensor nodes, $E_{m}$ is the full charged energy, and $\alpha$ is the weight factor used to adjust the importance of the remaining energy and localization error.

The cost function for slave node selection $C_{b e}$ (Equation 4.3) is transformed into $C_{b e, e}$ given by,

$$
C_{b e, e}=\left(\beta+(1-\beta) \frac{E_{r}}{E_{m}}\right) C_{b e}
$$

where, $E_{r}$ is the remaining energy of the candidate slave node, $E_{m}$ is the full charged energy, and $\beta$ is the weight factor used to adjust the importance of the remaining energy 
and localization error. These new cost functions make the sensor node selection process energy-aware and provide a method to balance target tracking performance and sensor network lifetime.

\subsection{The Localization and Tracking Algorithm}

The developed energy-efficient distributed self-organization algorithm has three major stages, namely, the initialization stage, the target tracking stage and the localization sensor group update stage. In the initialization stage, a rough estimate of the target location is obtained, and the localization sensor group is initialized using this location estimation. Target localization using the selected localization sensor group is performed in the target tracking stage. In addition, the Kalman filter based tracking method is employed in this stage for the target state estimation and prediction. Updating of the localization sensor group according to the target dynamics is performed in the localization sensor group update stage. The algorithm is shown in Figure 4.2 and the detailed description follows.

\subsubsection{Initialization of the Localization Sensor Group}

The node selection process described in Section 4.2 requires an estimate of the target location to perform the node selection. Thus, the formation of the localization sensor group requires an initial estimation of the target location. This initial estimation is obtained using multiple initial localization sensor groups formed using the closest node selection method. 


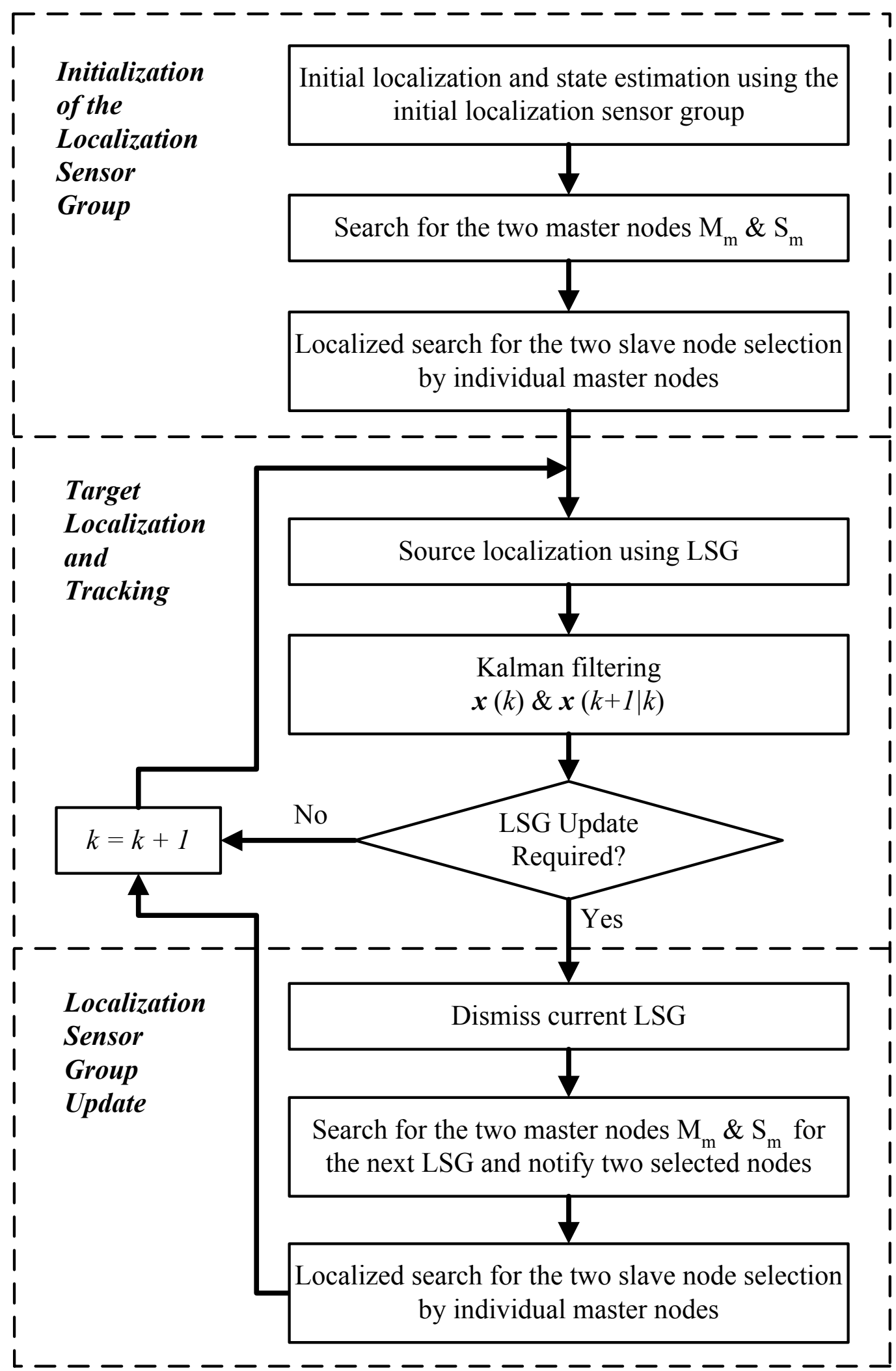

Figure 4.2 The Energy-Efficient and Distributed Self-Organization Algorithm 


\subsubsection{Initial Four Closest Nodes Localization Sensor Groups Formation}

In the closest nodes selection method, the " $n$ " sensor nodes in the local neighborhood that have the highest received acoustic signal strength, which are located in the far field, are selected for a localization sensor group. In our work, we have selected the four closest nodes for an initial localization sensor group. The localization sensor groups are formed in the following manner. Once a node detects a target with received signal strength within a certain range, it decides to become a candidate for a group head. Then it sends out a request message for other nodes in its one-hop neighborhood to join its group. There will be a contention between the neighboring nodes to become the group heads. The contentions for the group heads are handled using a backoff scheme, where backoff time is calculated based on the received acoustic signal strength. In this scheme, each candidate group head node will backoff for some time interval before sending the request message to join its group. The backoff time depends on the received acoustic signal strength and the nodes with higher received acoustic signal strength will have a lower backoff time. If a candidate head node receives a join request message from another candidate head node during this backoff time, it will give up the head state. Once the request message is sent, the candidate head node waits for some time and selects the best three other nodes from the received replies and forms an initial four closest nodes (FCN) localization sensor group. When the group formation is complete, the group head node will notify the selected member nodes. The notification also includes the time to start the tracking. There would be multiple such groups formed throughout the sensor network. The range of the received signal strength is decided off-line and is set in a 
manner so that these groups will be formed in the far field, close to the far field boundary.

\subsubsection{Initial Location Estimation}

When the selected sensor nodes receive the notification, they will start to track the target by sending the received acoustic signal to the group head node on time intervals defined by the head node. The head node will collect these signals and calculate the corresponding TDOA value estimations. The target location estimations can be achieved using these estimated TDOA values by the localization method discussed in Section 3.1. In addition, once the initial localization sensor group formation is complete, the head nodes will broadcast a head notification message before the tracking starts. When the neighboring nodes receive this message, they will broadcast it again. When a head node receives this message, it will add the message originating head node to its neighboring head list. Thus, after first couple of time intervals from initial localization sensor group formation, a rough estimation of the target location and information about the neighboring group head nodes will be available at each group head node. After this, the neighboring group head nodes exchange the TDOA estimations and sensor group location information to refine the target location estimation.

\subsubsection{Localization Sensor Group Formation}

Once a refined target location estimation and state prediction is obtained, head node of the each initial four closest nodes localization group sends out a message with the estimated target location and state predictions information. This message is forwarded along the far field boundary, which is determined using the estimated initial target location. The following figure illustrates the forwarding path of these messages. 


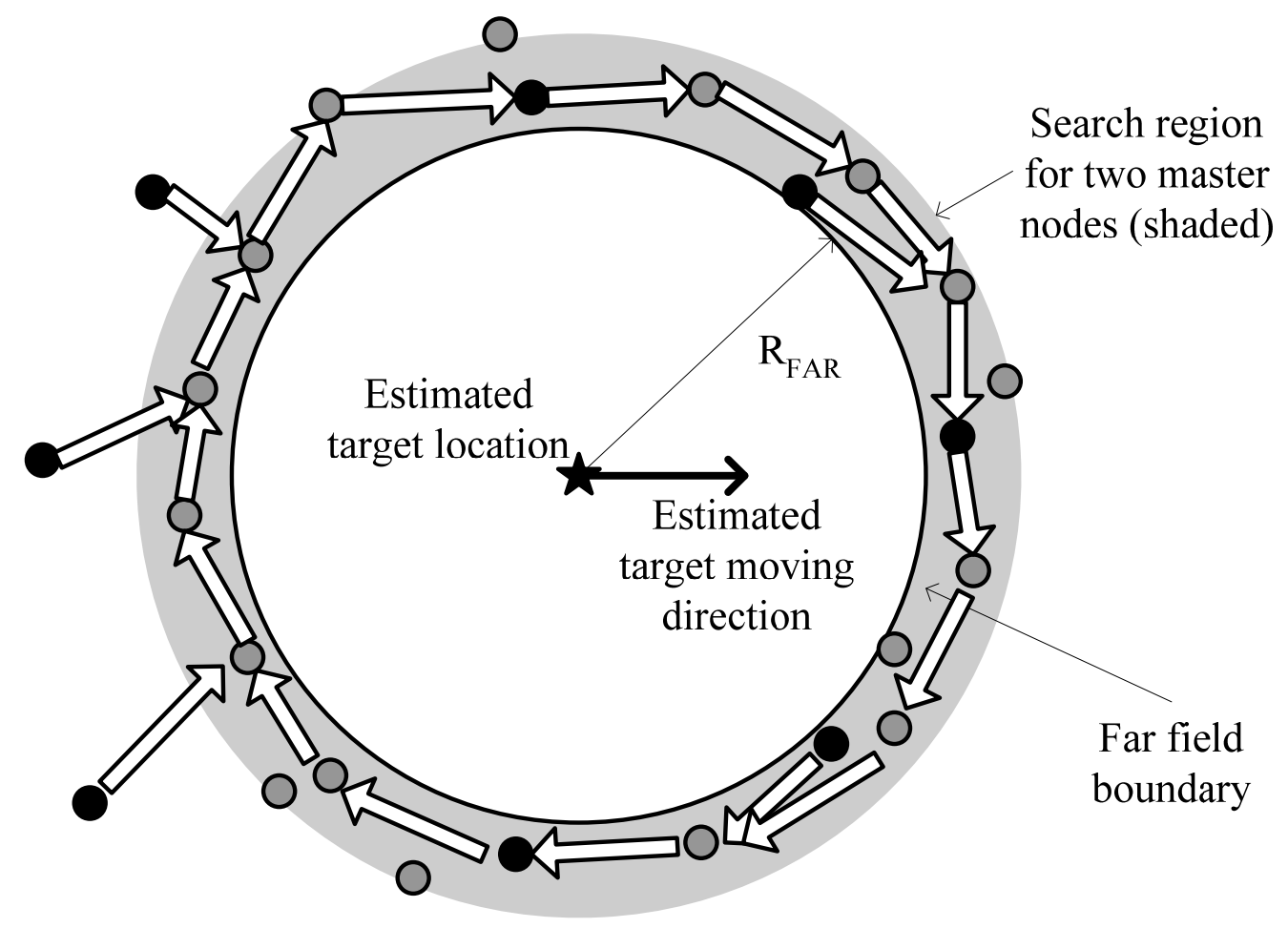

O Sensor nodes $\bigcirc$ FCN group head nodes $\Rightarrow$ Message forwarding path

Figure 4.3 Forwarding Path of the Initial Target Location Estimation

When a node receives such a message it will check whether it has already forwarded a message from a head node with higher received acoustic signal strength. If so, it will discard the current message. Otherwise, it will forward the message along the path after adding its own node information into the message. Thus, only the message from the FCN group head node with highest received acoustic signal strength will travel the full path. The other messages will be eliminated by the nodes along the path. Then, finally when a message completes traversing the path along the far field boundary, the last node that receives the message performs the master node selection. It selects the two master nodes using the information contained in the message and notifies the two selected master nodes. The message will contain the target location estimation, target 
state prediction and the information about the nodes in the region around the far field boundary, which the message traversed. With this information, the two master nodes are selected using the resource aware node selection process described in Section 4.2. Then, the two master nodes will individually perform the slave node selection step of the node selection process described in Section 4.2 and select and notify the two slave nodes. Once both the master nodes select their respective slave nodes, the localization sensor group initialization is complete.

\subsubsection{Target Localization and Tracking}

The localization sensor group will perform the target localization and tracking using the localization and tracking methods described in Section 3.1 and Section 3.3.

\subsubsection{TDOA Estimation}

Once the tracking process starts, each sensor node in the localization sensor group will record the received acoustic signal. Then, the two slave nodes will send their acoustic signals to the respective master nodes. Upon receiving the message, the master nodes will obtain an estimation of the TDOA using the received acoustic signal and its own acoustic signal.

\subsubsection{Target Localization}

After calculating the TDOA estimation, the master node of the slave sensor pair will send its TDOA estimation and the location information of the slave sensor pair to the master node of the master sensor pair. When the master node of the master sensor pair receives this message, it will combine this information with the TDOA estimation and the location information of the master sensor pair, and calculate the target location estimation using the localization method described in Section 3.1. 


\subsubsection{Target Tracking}

Once the target location estimation is obtained, this estimation is used as the measurement to the Kalman filter based tracking method. Using this measurement, the filtered current state and the predicted next state of the target are calculated. Specifically,

the Kalman filter will generate the filtered estimate of the current target state $\boldsymbol{x}(k)$, which contains the location and the velocity of the target. In addition, the next target state, $\boldsymbol{x}(k+1 \mid k)$ will be generated. This prediction will be used for the localization sensor group update to match the target dynamics.

\subsubsection{Localization Sensor Group Evaluation}

Since the localization accuracy is closely related to the geometry between the sensors and the target, the localization performance of current localization sensor group may degrade as the target moves. Hence, the suitability of the localization sensor group should be reevaluated after each tracking interval and if required, it should be updated to match the target movement in order to maintain satisfactory tracking performance. This process is carried out in the following manner.

When the acoustic signal from the target is captured, each sensor node in the localization sensor group monitors the received acoustic signal strength. If the signal strength is above a predefined threshold, the sensor node assumes that the sensor is not far enough from the target and the far field assumption is violated. If a slave node detects that it is violating the far field condition, then it will notify the corresponding master node when it sends the captured acoustic signal to master node. The master node of the slave sensor pair notifies the detected far field condition violations in the slave group (either a violation notified by the slave node or its' own violation detected based on the received 
acoustic signal strength) to the master node of the master sensor pair. After the target tracking step, the master node of the master sensor pair checks whether any sensor node is the group has reported a far field condition violation and if so, decides to update the localization sensor group. This decision is based on the condition that if any node is not in the far field of the target, then the group is not suitable for performing the localization.

If no far field condition violations are reported by the sensor nodes, the master node of the master sensor pair will check whether the current localization sensor group will violate the far field assumption in the next step. For this, after obtaining the next state prediction $\boldsymbol{x}(k+1 \mid k)$, the distance from each sensor node in current localization sensor group to the predicted next location of the target is calculated. Then, if any of these distances is less than $R_{F A R}$, the master node of the master sensor pair will predict a far field condition violation in the next step and decides to update the localization sensor group.

If far field condition violation is neither reported nor predicted, the master node of the master sensor pair will then evaluate the suitability of the localization sensor group for performing the localization in the next step. First, it will calculate the bearing estimation cost function values $\left(C_{b e, e}\right.$ defined in Equation 4.5) for master and slave sensor pairs. Then, using these values, the cost function $C_{\text {geo,e }}$ (defined in Equation 4.4) will be reevaluated for the current localization sensor group using the predicted next target location. If the cost function $C_{g e o, e}$ value increases over a certain threshold $\left(C_{T H}\right)$ compared to the previous cost function value, then the master node of the master sensor pair will decide to update the localization sensor group. The predefined threshold value, $C_{T H}$ can be used for the balancing of the target tracking quality and the localization sensor 
group update frequency. This is another parameter that can be adjusted to balance the tracking performance and the resource usage for tracking.

\subsubsection{Localization Sensor Group Update}

If the master node of the master sensor pair decides to update the localization sensor group, it will initiate the localization sensor group update by dismissing the current localization sensor group. Then, the search for the two master nodes of the next localization sensor group is initiated.

\subsubsection{Desirable Geometry Formation of Master Sensor Nodes}

According to our analysis in Section 4.2, the near-optimal localization sensor group will be located adjacent to the boundary of the far field. Thus, the two near-optimal master nodes too will be located in the same region. A possible optimal geometry formation of master sensor nodes is illustrated in Figure 4.4.

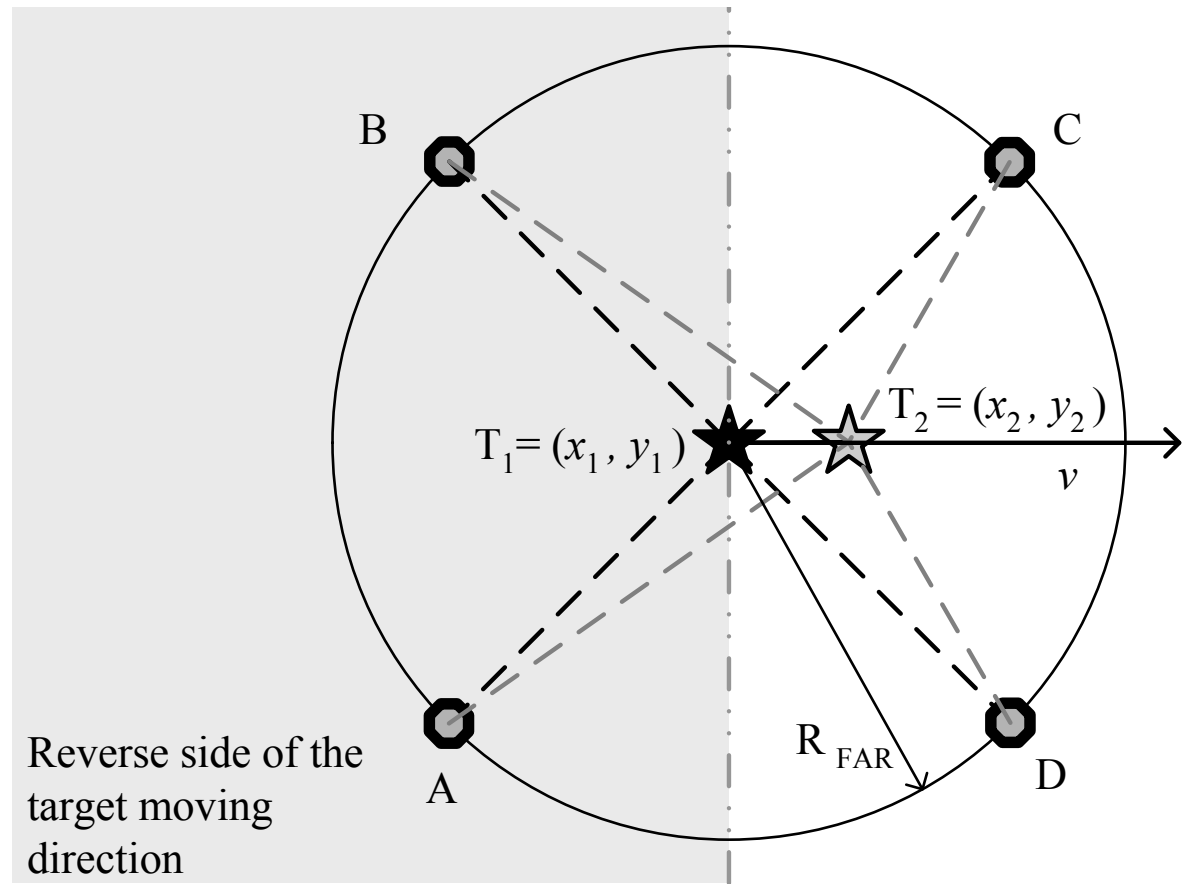

Figure 4.4 Master Sensor Node Locations 
Here, four potential sensor nodes are marked as A, B, C, and D with respect to the current target location $T_{1}$ and future target location $T_{2}$. The velocity of the target is denoted by $v$ and $R_{F A R}$ is the threshold distance required for satisfying the far field condition. The four candidate master node pairs ((A, B), (B, C), (C, D) \& (A, D)) are optimal selections with respect to the current target location $\mathrm{T}_{1}$.

Since the sensor network is densely deployed, each of these candidate master node pairs is equally attainable. In addition, if we consider the criterion for the two master nodes selection (cost function $C_{g e o}$ specified in Equation 4.2), all these candidate master node pairs will be optimal. Actually the positions of these nodes can be moved along the (circular) far field boundary as long as the relative geometry of the nodes and the target location are preserved. Although these candidate master node pairs can achieve almost the same cost function value in terms of $C_{\text {geo }}$, they differ greatly if we consider the group updating in tracking a moving target.

If we consider the candidate master node pairs (A, B) and (B, C), the baseline of the candidate master node pair (A, B) is perpendicular to the target moving direction and the baseline of the candidate master node pair $(\mathrm{B}, \mathrm{C})$ is parallel to the moving direction of the target. Although these two candidate master node pairs have the same $C_{\text {geo }}$ cost function value when the target is at position $\mathrm{T}_{1}$, the angle $\angle B T_{2} C$ will decrease rapidly compared to the angle $\angle A T_{2} B$. This will cause the tracking performance of the candidate master node pair (B, C) to degrade faster than the candidate master node pair (A, B). In addition, the sensor node $\mathrm{C}$ is in the front of the target moving direction. Thus, as the target moves, the distance between the sensor node $\mathrm{C}$ and the target will decrease and this can cause violation of the far field condition. In contrast, the nodes A and B are in the 
reverse side of the target moving direction and are less likely to cause a far field condition violation. Thus, it is evident that the candidate master node pair $(\mathrm{B}, \mathrm{C})$ is more likely to be updated frequently. This analysis is also valid for candidate master node pair (A, D).

Meanwhile, if we consider the candidate master node pairs (A, B) and (C, D) the baseline of the candidate master node pair (C, D) is also perpendicular to the moving direction of the target. Even though the deviation of angle $\angle A T_{2} B$ from the desired $90^{\circ}$ will be lower compared to that of angle $\angle C T_{2} D$, the difference can be considered very small (in the initial stages). In addition, the distance from future target location $\mathrm{T}_{2}$ to the sensor nodes $\mathrm{C}$ and $\mathrm{D}$ are smaller compared to that of sensor nodes $\mathrm{A}$ and $\mathrm{B}$. Thus, it may seem like that the candidate master node pair $(C, D)$ is a better choice than candidate master node pair (A, B). However, both the sensors of the candidate master node pair (C, D) are in the front of the target moving direction. Thus, it is highly possible that when the target moves the far field assumption condition will be violated causing frequent localization sensor group updates.

According to the above analysis, the candidate master node pairs similar to (A, B) have the most desirable geometry formation for tracking moving target as they result in more stable localization sensor groups. Therefore, when selecting the master node pairs for next localization sensor group, focus will be given to selecting such pairs. This is achieved by selecting the two master sensor nodes from the reverse side of the target moving direction, highlighted in Figure 4.4. 


\subsubsection{Search for the Two Master Nodes}

The search for the two master nodes is initiated by the current master node of the master sensor pair. The search request message is in the direction parallel to the predicted moving direction of the target by the two master nodes. The rationale behind the selection of such a path is, since the current master and slave sensor pairs are nearly optimal for the current target location, if the current sensor pairs can follow the target movement, it is highly possible that they will remain the best sensor pairs for the predicted target location. Thus, the search for the best sensor pair for the predicted target location can be limited to the path parallel to the movement of the target. In addition, to minimize the effect of sub-optimality that may be present in the previous sensor group and the target movement prediction error, the search request message is forwarded along the outside border of the far field boundary of the predicted target location. This will also provide more flexibility in selecting the sensor pair with energy considerations. The search message forwarding direction or the master node of the master sensor pair is illustrated in Figure 4.5. Master node of the slave sensor pair will forward the request in a similar path.

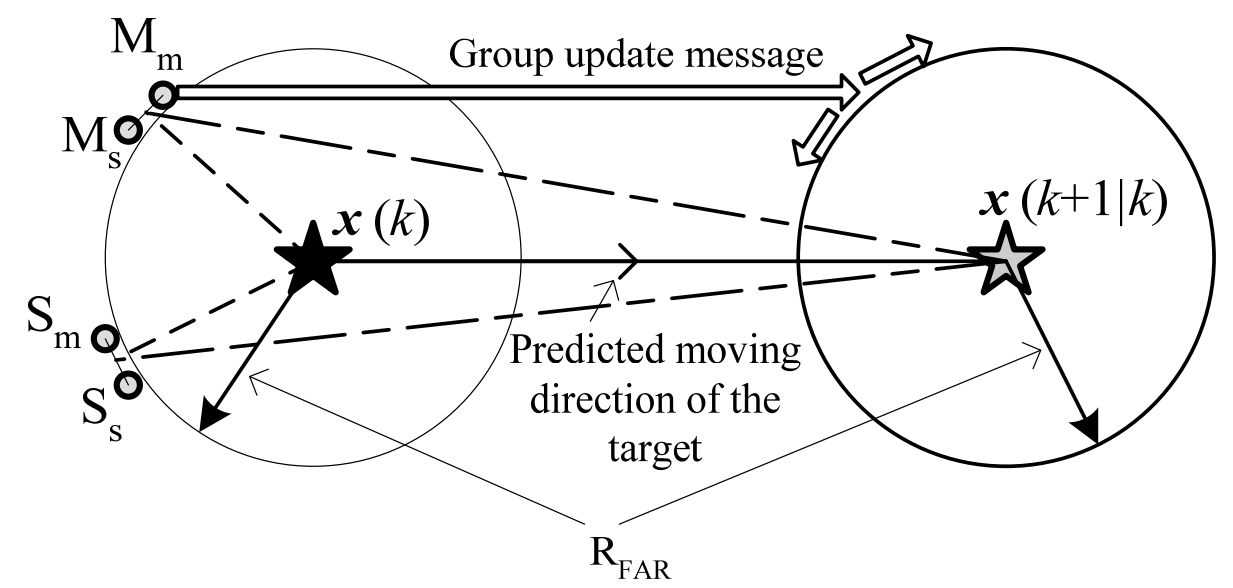

Figure 4.5 Localization Sensor Group Update 
Here, $M_{m}, M_{s}, S_{m}$ and $S_{s}$ represent the master and slave nodes of the master and slave senor pairs of the current localization sensor group. $R_{F A R}$ is the threshold distance required for satisfying the far field condition. $\boldsymbol{x}(k)$ and $\boldsymbol{x}(k+1 \mid k)$ represent the current and next (predicted) target states and the stars indicate the current and next (predicted) target locations.

Upon receiving the search request messages, the nodes will add their own locations and energy usage information to the messages and forward them. Once the forwarding limit is reached, the last nodes that receive the messages will send the accumulated information present in the messages to the master node of the master sensor pair. Then, the master node of the master sensor pair will select the two best master nodes based on the resource aware node selection process described in Section 4.2 and notify the two selected nodes. The master sensor node that is closer to the target is designated as the master node of the master sensor pair. The current target state estimation and the details of the Kalman filter are forwarded to this node along with the notification.

\subsubsection{Localized Searches for the Two Slave Nodes}

The two master nodes will select the respective slave nodes from their one-hop neighborhoods. Thus, the two master nodes will individually perform the slave node selection step as described in Section 4.2, and select and notify the two slave nodes. This completes the localization sensor group update and after the localization sensor group update is completed, the new localization sensor group will take over the target tracking task. 


\subsection{Message Passing for Energy-Efficient and Distributed Self-Organization}

This section provides the implementation details of the energy-efficient and distributed self-organization algorithm. The message passing protocol developed to achieve the distributed self-organization is discussed in detail. Thus, the details of the message contents and the actions performed upon the receiving of the messages are elaborated in this section. In addition, the important parameters of the message passing algorithm are also discussed.

\subsubsection{Initialization of the Localization Sensor Group}

Initially, all the nodes are sensing the environment for possible presence of a target. If a node perceives the received acoustic signal strength within a certain range, it initializes the localization sensor group formation. The range of the signal strength is defined in the following manner. Signal strength expected at the far field boundary $\left(R S S_{\text {High }}\right)$ is the upper bound for the range. In addition, this value can be reduced slightly to cater for the inaccuracies caused by environmental conditions. The lower bound of the range $\left(R S S_{\text {Low }}\right)$ is the expected signal strength at a distance equal to the far field range distance plus the communication range of the sensor nodes.

\subsubsection{Initial Four Closest Nodes Localization Sensor Groups Formation}

Once a node detects a target with received signal strength within the above

described range, it decides to become a candidate for the group head node of the four closest nodes group. Then, it calculates its backoff time $\left(T_{\text {BackOff }}\right)$ as follows.

$$
T_{\text {BackOff }}=T_{B O, M A X}\left(\frac{R S S-R S S_{\text {Low }}}{R S S_{\text {High }}-R S S_{\text {Low }}}\right)
$$


where $T_{B O, M A X}$ is the predetermined maximum backoff time value that will depend on the sensor node density, application requirements and the one-hop communication delay. RSS is the received signal strength of the node and $R S S_{\text {High }}$ and $R S S_{\text {Low }}$ are the limits of the acoustic signal strength range discussed above. Then, the node backoff for $T_{\text {BackOff }}$ and broadcasts a message to its one-hop neighborhood with a request to join its group. However, if a node receives an initial group join request message during its backoff time, it relinquishes its group head state and replies to the request it received. The format of this initial group join request (IGJReq) message is shown below.

IGJReq $=($ Head ID; Head Location; Head Received Signal Strength; Head Neighboring Node Count)

where "Head Neighboring Node Count (HeadNNC)" is the number of nodes in the one-hop neighborhood of the group head node, according to its knowledge. When a node receives initial group join request message, it first evaluates its received signal strength to determine whether it is below the received signal strength of the head node. If it is above this limit the node will simply discard the message. Else, it will check whether the distance between the head node and itself is less than the maximum allowable sensor separation distance $\left(L_{T H}\right)$. If it is less than $L_{T H}$, the node will discard the message. Otherwise, it will evaluate its suitability to join the group by evaluating the cost function;

$$
C_{I G}=\frac{R S S}{R S S_{\text {Head }}} \times \frac{d}{L_{T H}}
$$

Here, RSS is the received signal strength of the node and $d$ is the distance between the node and the group head node. $R S S_{\text {Head }}$ is the received signal strength of the head node. If 
this value is above a threshold, $\left(C_{I G, T H}\right)$, then the node will send the reply to the group head node. Otherwise, it will decide to send the reply with a probability of $P_{r}$.

$$
P_{r}=\max \left\{1, \frac{1.5 \times n}{H e a d N N C}\right\}
$$

where HeadNNC is obtained from the request message. $n$ is the group size of the closest node group, 4 in this case. The format of the initial group join reply message (IGJRep) is shown below.

\section{IGJRep $=($ Node ID; Node Location; Node Received Signal Strength $)$}

If a group head node, that has sent the initial group join request message, receives a request from another head node, the receiving head node will compare the received signal strengths of the two nodes. If the signal strength of the receiving group head node is lower, then it relinquishes its group head state and replies to the request it received. Otherwise, the request will be discarded.

After sending the initial group join request message, the group head node will wait for a predetermined time $\left(T_{I G S}\right)$ for the neighboring nodes to reply to the request and select the three nodes with highest received signal strength to complete the four closest nodes group. If the head node does not get enough replies, then it will relinquish its group head state. Once the group selection is complete, the group head node notifies its group members by sending initial group announcement (IGA).

$$
\begin{aligned}
\mathbf{I G A}= & (\text { Initial Group Nodes }(\text { Head ID, Node_1 ID, Node_2 ID, Node_3 ID }) \\
& \text { Tracking Start Time })
\end{aligned}
$$

where "Tracking Start Time" is the first time to start sending the received acoustic signal to head. 


\subsubsection{Initial Groups Discovery}

Once the initial four closest nodes group is formed, the group heads will obtain information about each other through flooding. To achieve this, the group heads will broadcast an initial group head notification message (IGHN) with time to live field set to a predefined time to live $\left(T T L_{I G H N}\right)$ value.

IGHN = (Head ID; Head Location; Head Received Signal Strength; Time to Live $)$ When a node receives this message, if it has received it earlier it will discard it. Otherwise it will reduce the TTL value of the message by 1 and rebroadcast it. If the receiving node is a group head node, then it will add the message originating head node to its neighboring head nodes list.

\subsubsection{TDOA Information Exchange for Initial Location Estimation}

The non-head nodes of the initial group will start to send the captured acoustic signal to the group head at the time specified by head node $\left(T_{T S}\right)$. They will send $N_{I T}$ number of such samples in time intervals of $T_{S}$ (sampling time). This initial group node acoustic signal message (IGNAS) has the following format.

IGNAS $=($ Node ID; Capture Time; Acoustic Signal $)$

When group head node receives this message, it will calculate the TDOA value for the sensor pair using its own acoustic signal and the received signal and store it. After all $N_{I T}$ samples are collected, it will send the location information of all the nodes in the group and the calculated TDOA estimations to the known neighboring head nodes. The message format is,

IGTE $=($ Head ID; Head Location; Noad_1Location; Noad_2 Location; Noad_3 Location; TDOA Estimations) 
where TDOA Estimations $\equiv\left[\begin{array}{cccc}\text { Capture Time 1 } & \text { TDOA1 } & \text { TDOA2 } & \text { TDOA3 } \\ \text { Capture Time 2 } & \text { TDOA1 } & \text { TDOA2 } & \text { TDOA3 } \\ \vdots & \vdots & \vdots & \vdots \\ \text { Capture Time } N_{I T} & \text { TDOA1 } & \text { TDOA2 } & \text { TDOA3 }\end{array}\right]$

After sending out the TDOA estimations, the head node will backoff for some fixed time interval $\left(T_{I G E}\right)$ to receive TDOA information from the other head nodes. Then it estimates the current location, and the next state, of the target using the TDOA information it receives.

\subsubsection{Search for Master Nodes}

In order to form the localization sensor group, all the initial group head nodes have to come to an agreement. Since there is no central control, this requires collaboration between all the group head nodes. However, all the group head nodes do not know about the existence of all other group head nodes. Therefore, to overcome this collaboration requirement, the localization sensor group is formed in the following manner. Once the target state estimation is obtained, each head node checks whether it is located in the far field of the target location estimation. If this condition is not met, that suggests that either estimation is inaccurate or the initial group does not satisfy the far field condition. Thus, the group head will discard its estimates. Otherwise, the group head nodes send out the initial location estimation forwarding message (ILEF) with the estimated target location and state prediction information along the far field boundary of the estimated target location.

\section{ILEF $=($ Head ID; Head Received Signal Strength; Current Target State; Generated Time; Kalman Filter Parameters; Time to Live; Node Details)} The "Time to Live" (TTL) of this message is set to $T T L_{I G T E}$ and it can be estimated by, 


$$
T T L_{\text {IGTE }}=\frac{2 \pi R_{F A R}}{R_{\text {Comm }}}
$$

Here $R_{F A R}$ and $R_{C o m m}$ are far field range distance and the one-hop communication range. The field "Node Details" contains the node location and the energy level of each node the message has reached. The "Kalman Filter Parameters" field contains the prediction error covariance matrix $(\boldsymbol{P}(k+1 \mid k))$ of the Kalman filter. When a node receives this message, first it checks whether it received a similar message with generated time in similar range. If it already received such a message, it compares the received acoustic signal strengths of the two head nodes. If the new message has a lower received acoustic signal strength, then that message will be discarded. This process eliminated the estimates generated by the group heads other than the group head that is located closest to the target. If the received acoustic signal strength is higher, the node checks whether it is in the far field of the target estimation. If it is in the far field of the target estimation, the node adds its location and energy information to the "Node Details" field. Then, the node decreases the $T T L$ value by one and forwards the message along the far field boundary. When, an initial location estimation forwarding message (ILEF) reaches $T T L$ value of ' 0 ', the node that receives the message will select the two master nodes from the information contained in the message, using the resource aware node selection process described in Section 4.2. The target state prediction for the next time interval $(x(k+1 \mid k))$ will be used in this master node selection process. Then, the master node that is closer to the target estimation is selected as the master node of the master sensor pair $\left(M_{m}\right)$ and other node is the master node of the slave sensor pair $\left(S_{m}\right)$. Then, these two master nodes are notified through the 
master sensor pair master node notification message (MSPMNN) and slave sensor pair master node notification message (SSPMNN).

MSPMNN $=\left(M_{m} I D ; S_{m} I D ; S_{m}\right.$ Location; Current Target State; Tracking Start Time; Kalman Filter Parameters; $C_{g e o, e}$ Value )

SSPMNN $=\left(S_{m} I D ; M_{m} I D ; M_{m}\right.$ Location; Current Target State; Tracking Start Time)

The field "Kalman Filter Parameters" is similar to that of initial location estimation forwarding message (ILEF).

\subsubsection{Localized Search for Individual Slave Nodes}

When the master nodes receive these messages, they will broadcast a slave node request message (SNReq) to its one-hop neighborhood. In addition, the master node of the master sensor pair will store the Kalman filter parameters and the cost function value for future use.

\section{SNReq $=($ Master Node ID; Master Node Location; Predicted Target Location; Master Node Neighboring Node Count)}

When the neighboring nodes receive this message, they will check whether they are in the far field of the target. If not, the message will be discarded. Otherwise, they will calculate their bearing estimation cost function $C_{b e, e}$. If this value is above a threshold value $\left(C_{b e, e, T H}\right)$, it will reply to the master node. Otherwise, the node will decide to reply with a probability $P_{s}$,

$$
P_{s}=\max \left\{1, \frac{s}{\text { Master Node NNC }}\right\}
$$

The format of this reply message (SNRep) is, 
SNRep $=\left(\right.$ Node ID $;$ Node Location; $C_{b e, e}$ Value $)$

After sending the slave node request message, the master node will wait some time $\left(T_{S N S}\right)$ for the neighboring nodes to reply. Then it will select the node with the highest $C_{b e, e}$ value as its slave node and notify it.

SNNot $=($ Node ID; Tracking Start Time $)$

\subsubsection{Target Localization and Tracking}

When the selected slave nodes receive the notification messages, they will start tracking and sending the captured acoustic signals to the respective master nodes. This will begin at the tracking start time specified by master node and will be done in intervals of $T_{s}$ (sampling time) until the localization sensor group is dismissed. The message format of the slave node acoustic signal (SNAS) is,

SNAS $=($ Capture Time $;$ Acoustic Signal; Slave Node Energy Level; Is Slave Node in Far Field)

When the master node receives the acoustic signal from the slave node, it will estimate the TDOA value using that signal and its own acoustic signal. After calculating the TDOA value, the master node of the slave sensor pair will send the estimated TDOA value and the energy levels of the two nodes of the slave sensor pair, to the master node of the master sensor pair. In addition, this message will optionally contain the location of the slave node of the slave sensor pair (if this is the first target tracking of this group). This slave group TDOA estimation message (SGTE) will have the following format.

SDTE $=($ Capture Time $;$ TDOA Estimation; Master Node Energy Level; Slave Node Energy Level; Is Master Node in Far Field; Is Slave Node in Far Field [; Slave Node Location]) 
The fields "Is Master Node in Far Field" and "Is Slave Node in Far Field" indicate whether the respective nodes received a signal strength that would indicate a violation of the far field assumption.

\subsubsection{Localization Sensor Group Update}

If the master node of the master sensor pair decides to update the localization sensor group, it will dismiss the current localization sensor group. First it will send the master node dismiss message (MND) to the master node of the slave sensor pair.

$$
\mathbf{M N D}=(\text { Dismiss Time } ; \text { Next Target State Prediction } ; \text { LSGUReq Expire Time })
$$

The predicted next target state and expire time of the localization sensor group update request message (LSGUReq) are included in this message so that the master node of the slave sensor pair too can initiate the search process for the next master nodes. Upon receiving the dismiss message, the master node of the slave sensor pair will send the slave node dismiss message (SND) to its slave node.

$\mathbf{S N D}=($ Dismiss Time $)$

Also, the master node of the master sensor pair too will send the dismiss message to its slave node. Upon receiving the dismiss message, the slave nodes will give up their slave node roles. After dismissing slave nodes, each master node will independently start the search for the next master nodes by initiating a localization sensor group update request message (LSGUReq).

LSGUReq $=\left(M_{m} I D ; M_{m}\right.$ Location; Next Target State Prediction; LSGUReq Expire Time; Time to Live; Node Details)

Initially, the node details will be empty. However, it will have the data format as the "Node Details" field and as the message passes forward in the path described in Section 
4.3.3, the nodes will add their information to the message. The time to live value will be set to a threshold value $\left(T T L_{L S G U}\right)$, which will depend on the communication latency, far field range distance $\left(R_{F A R}\right)$, communication range of the nodes $\left(R_{C o m m}\right)$ and the real-time requirements of the algorithm. After initiating this message, the master node of the slave sensor pair will give up its role. When a node receives this message, it will check whether it is in the far field of the target and if so will add its information to the "Node Details" field. Then, it will reduce the TTL value by one and forward it along the path described in Section 4.3.3. When the TTL value becomes " 0 ", the node that receives the message will send the node information present in the message to the master node of the master sensor pair.

\section{LSGURep $=($ Node Details $)$}

In addition, an expire time is assigned to the LSGUReq message, so that the timeliness of the localization sensor group update process can be maintained. In forwarding the LSGUReq, each node will check whether expire time of the message has elapsed. If so, rather than forwarding it, the node will send the node details contained in the message to the master node of the master sensor pair.

After sending the LSGUReq, the master node of the master sensor pair will backoff for some time $\left(T_{L S G S}\right)$ to receive the replies and then will select the next master nodes using the node information it receives from replies. In addition, the master node that is closest to the target is selected as the master node of the master sensor pair. The above described expiring of the LSGUReq message is introduced to maximize the chance of receiving the LSGURep (may be with partial information) before the selection is carried out, especially when unexpected communication delays occur. After the two 
master nodes are selected, the current master node of the master sensor pair notifies the new master nodes (through MSPMNN and SSPMNN) and gives up its role. The new master nodes will carry out the localized search process for individual slave node selection (described in Section 4.4.1.5) to complete the localization sensor group update.

\subsection{Energy Conservation}

Incorporation of energy conservation mechanisms that can reduce the energy usage is vital for the extended lifetime of the energy limited sensor networks $[13-16][106][107]$. One approach for reducing energy usage is the selection of less power consuming processing and communication hardware. Although this approach can reduce the energy usage, it has limits on the reduction of energy usage. Thus, effective power management is very important in achieving extended network lifetime. Using simple

processing algorithms, reducing the communication requirements, using energy aware node selection for target tracking and use of energy aware routing and MAC layer protocols that consider node energy levels in communication mechanisms are a few examples of such power management mechanisms. Another method that has attracted interest is exploiting the densely deployed and the event and location centric nature of sensor networks to improve energy efficiency by incorporating efficient sleep-wakeup management mechanisms.

In the designed self-organization algorithm a simple and resource efficient localization method is employed for target localization. In addition, the algorithm is developed in a distributed manner. In order to minimize the communication requirements, a geographically local resource aware node selection method is employed. To further improve the energy conservation, a sleep-wakeup management scheme is 
incorporated to the self-organization algorithm. The sleep-wakeup management mechanism is implemented using the combination of energy efficient low latency MAC protocol proposed by Wan et al. [16][17] and a novel cross layer wakeup adjustment algorithm. This section provides the details of the sleep-wakeup management mechanism.

\subsubsection{Energy Conservation Using Sleep-wakeup Management Schemes}

Wireless sensor networks are usually densely deployed and this can be effectively utilized for achieving energy conservation. Krishnamachari et al. [108] explained that phase transition phenomena are present in wireless sensor networks. Specifically, they pointed out that, "There are many contexts in distributed wireless networks where there is a critical threshold, corresponding to a minimum amount of the communication effort or power expenditure by individual nodes, above which a desirable global property exists with high probability" [108]. This provides the notion that, for successful operation of densely deployed wireless sensor networks only a subset of active nodes is sufficient. Thus, the densely deployed nature of the wireless sensor networks is exploited by many researchers, to reduce the energy waste by incorporating sleep-wakeup management mechanisms into the power management [13-16][106].

Moreover, interesting events such as presence of a target are rare in wireless sensor networks [13][106]. However, when an interesting event occurs, often the sensor network has to detect the event and respond to it in real-time. Therefore, in wireless sensor networks, the sensor nodes are often waiting in idle mode for such events to occur. Energy waste caused by this idle listening of sensor nodes is a major source of energy waste in wireless sensor networks [13]. In addition, the traffic in the sensor network is usually generated by such events and hence is geographically and temporarily correlated 
to these interested events [13]. Thus, handling such events and the traffic requirements of the wireless sensor networks can be successfully supported using only a subset of nodes in the sensor network which are located in close proximity to interesting events. Thus, by keeping only the required set of nodes awake and making the other nodes in the network sleep, considerable energy savings can be achieved. In addition, the geographically and temporarily correlated nature of events and traffic can be considered in activating nodes to achieve effective power management.

However, the power management may have adverse effects such as tracking inaccuracies, packet losses and increased communication latency. Thus, the successful power management will require support from the tracking algorithm and the underlying communication mechanisms (MAC and routing protocols) so that these effects can be either eliminated or minimized to achieve satisfactory tracking performance. Therefore, an energy efficient low latency MAC protocol and energy-aware anycast based message forwarding scheme proposed by Wan et al. [16][17], which support sleep-wakeup management of sensor nodes, is incorporated into the self-organization algorithm to further improve the energy efficiency. The details of the energy efficient MAC protocol and energy-aware message forwarding scheme and the proposed cross layer power management scheme are provided next.

\subsubsection{Energy Efficient and Latency Sensitive MAC Protocol}

Motivated by the phase transition phenomena explained by Krishnamachari et al. [108], the MAC layer protocol applies an opportunistic sleep scheme to achieve the energy efficiency. A wakeup probability is utilized to maintain the required number of active nodes and manage the network connectivity and the performance. Each node 
adapts to the changes in the local network traffic conditions by dynamically adjusting the wakeup probability. In addition, an anycast based data forwarding mechanism is proposed to reduce the end-to-end communication latency.

The MAC protocol is designed for wireless sensor networks with stationary nodes where multi-hop communication is employed for data delivery. In addition, it is assumed that the nodes are time synchronized and know their locations. An introduction to the major components of the protocol is provided here. More details about the MAC protocol and the energy saving and latency characteristics of the protocol can be found in [16] and [17].

\subsubsection{The Opportunistic Sleep Scheme}

In the proposed sleep scheme, the channel is divided into same length time slots and each node maintains its own wakeup probability depending on the local network condition and the performance expectations. In addition, the length of the time slot can be changed according to the application requirements. At the beginning of the time slots, each node decides to enter sleep or active state depending on its own wakeup probability. Thus each node will follow an alternating sleep and active pattern based on its own wakeup probability. This idea is illustrated in the following figure (Figure is a modified version of Figure 3.2 of [17]).

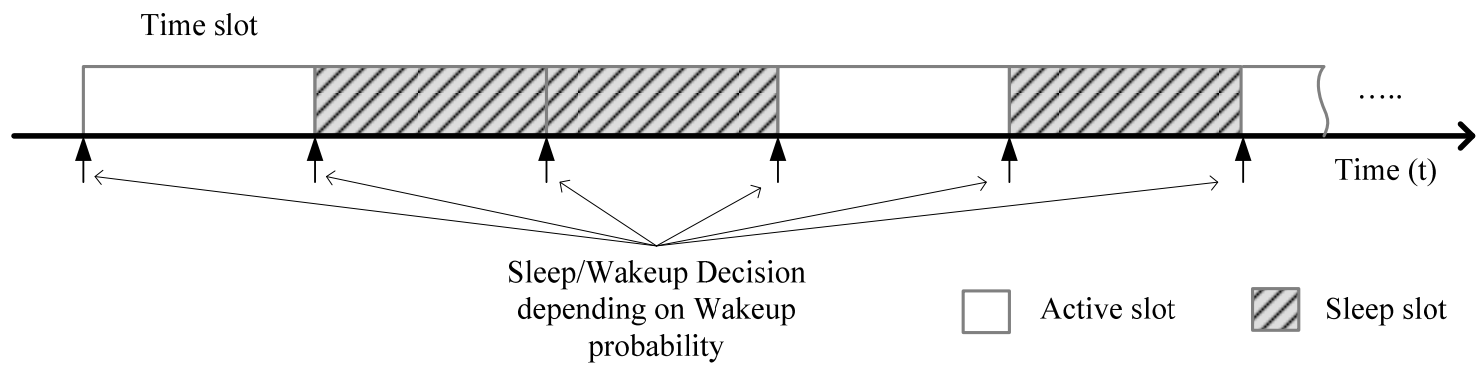

Figure 4.6 Opportunistic Sleep/Wakeup Scheme of MAC Protocol 
The wakeup probability determines the number of active nodes in the network and thus, the connectivity of the network. Higher wakeup probability will result in higher degree of connectivity while reducing the energy conservation. Thus, the wakeup probability should be dynamically adjusted by the sensor nodes to maintain the desired connectivity requirements of the network while maximizing the energy savings. The wakeup probability is dynamically adjusted by individual sensor nodes based on the local network condition, which is heuristically determined by monitoring the local traffic condition of the network and the performance expectations specified by the application layer. In this scheme, if no suitable forwarding node is discovered, it will cause increase in the wakeup probability assuming that higher degree of connectivity is required. However, if a collision is detected or the perceived packet loss rate is less than the desired threshold packet loss rate, it will assume that degree of connectivity is above the required threshold. Thus, the wakeup probability will be reduced to achieve better power savings.

\subsubsection{The Anycast Based Data Forwarding Scheme}

In order to minimize the effect of sleep management on communication latency and to improve the communication reliability, an anycast based packet forwarding scheme is introduced in the MAC protocol. In this forwarding scheme, the best node to forward the packet is chosen from the nodes in the direction towards the destination node. The selection is based on the geographical information and the remaining energy of the individual nodes.

Initially, the sender anycasts a request-to-send (RTS) message to a selected area which is determined by the forwarding trajectory pointing to the best direction toward the 
destination location. The selected forwarding area and the selection of the forwarding node are illustrated in Figure 4.7 (Figure is a modified version of Figure 3.6 in [17]).

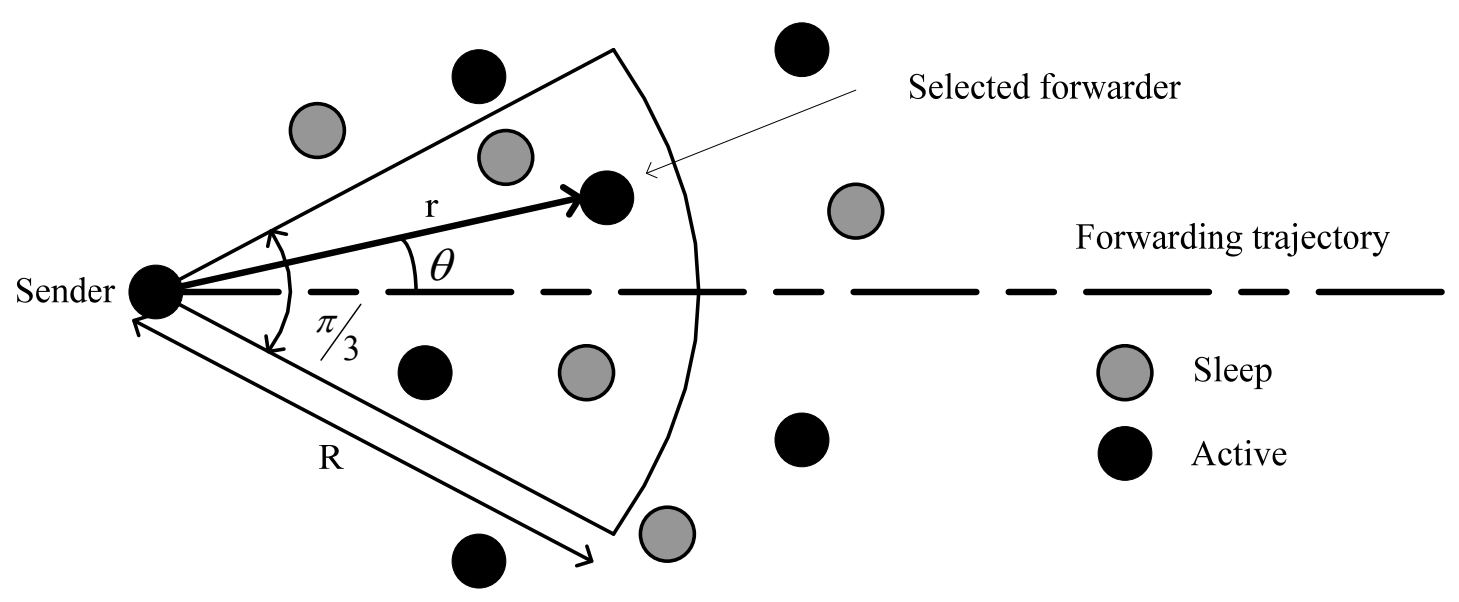

Figure 4.7 Selecting Forwarders for Anycast Based Data Forwarding Scheme

As it can be seen, the sector with central angle of $\pi / 3$ and radius $R$ (communication range) towards the forwarding trajectory is the selected forwarding area. The active nodes in this area are the candidate forwarding nodes. The RTS message contains the location information of the sender and destination nodes for the forwarding trajectory determination. Once a node receives this RTS message, first it will determine whether it is a candidate node by calculating the forwarding area and comparing it with its own location. If it is not a candidate node, it will go to sleep. The candidate nodes will calculate their priorities by evaluating the following cost function [17].

$$
C=\mu \frac{E_{r}}{E}+(1-\mu) \frac{r \cos \theta}{R}
$$

Here, $\mu$ is the weighting factor which balances the energy left in node and the geographic optimality of selected forwarder. $E_{r}$ is the remaining energy of the node, $E$ is the full charge energy and $r$ and $\theta$ are shown in Figure 4.7. After calculating the cost function, the nodes will backoff for a certain time interval depending on the priority. Then, the 
node with the highest priority will send its clear-to-send (CTS) message first. The other candidate nodes that hear this CTS message will discard their CTS messages and go to sleep. Once the sender receives the CTS message, it will send the packet to the selected forwarder and the forwarder will reply with an acknowledgement (ACK) message. This process will continue until the destination is reached.

In order to further reduce the end-to-end delay and improve energy efficiency, the location information of the sender and the destination nodes are included in the CTS message. Thus, the nodes that overhear the CTS message will determine whether they will be in the future forwarding trajectory and if they are in the future forwarding trajectory, they will decide to stay awake when the current transmission is complete. Moreover, the ACK messages which should be sent by the selected forwarding nodes at the end of each forwarding step are eliminated. This is achieved by letting the RTS message of the current forwarding step act both as the RTS message for the current step, and the ACK message for the previous step. This will reduce the transmission latency and the control overhead of the scheme resulting in improved energy efficiency.

\subsubsection{Cross Layer Power Management}

\subsubsection{Rationale for Cross Layer Power Management}

The adaptive adjustment of the wakeup probability from the MAC layer is mainly based on the local network condition, which is estimated by monitoring the presence of forwarding nodes, packet loss rate, and the presence of collisions in the network. Hence the wakeup probability adjustment is reactive to the network traffic conditions. Thus, when used in our target tracking application, the wakeup probability adjustment will be 
reactive to the communication requirements of the tracking algorithm. This could introduce a delay to the real-time target tracking.

Moreover, it is well known that the communication traffic patterns of the sensor networks are event and location centric [13]. This is especially true for the target tracking applications, where majority of traffic is generated by the interesting events such as detection of a target [13]. Thus, intuitively the combination of network condition and the knowledge about these events will allow a better adjustment of wakeup probability which will result in improved energy efficiency. In addition, the knowledge of these events will enable the adjusting of wakeup probability proactive to the requirements of tracking algorithm which will provide better communication facilities for real-time target tracking. However, only the application layer has the capability to identify these interesting events and the networking layers (MAC layer and routing layer) have the network status information. Therefore, a cross layer design, which combines event information from the application layer as well as the network condition from the MAC layer, is used in the proposed power management scheme of the energy-efficient distributed self-organization algorithm. This will enable the proactive adjustment of the wakeup probability by considering the event information as well as the network conditions. Moreover, as the wakeup probability adjustment is proactive, wakeup probability can be further reduced

during the idling time period in order to achieve increased power savings. The proposed cross layer wakeup probability adjustment scheme is presented next.

\subsubsection{Cross Layer Wakeup Probability Adjustment Scheme}

The major design goal of the cross layer wakeup probability adjustment is to combine the event information from the application layer and the network conditions 
from the MAC layer to achieve high energy efficiency while ensuring satisfactory tracking performance. The estimated application level requirements and the estimated sensor network local conditions are considered when sensor node wakeup probability is adjusted using this scheme.

In the application layer, the nodes are categorized based on the likeliness of the node participating in the target tracking process during the next few time intervals. According to this assessment, the node is classified into one of the four classes, namely "Very Low Important", "Low Important", "Medium Important" and "Highly Important". Following figure illustrates the category each node should be assigned depending on the region it is located.

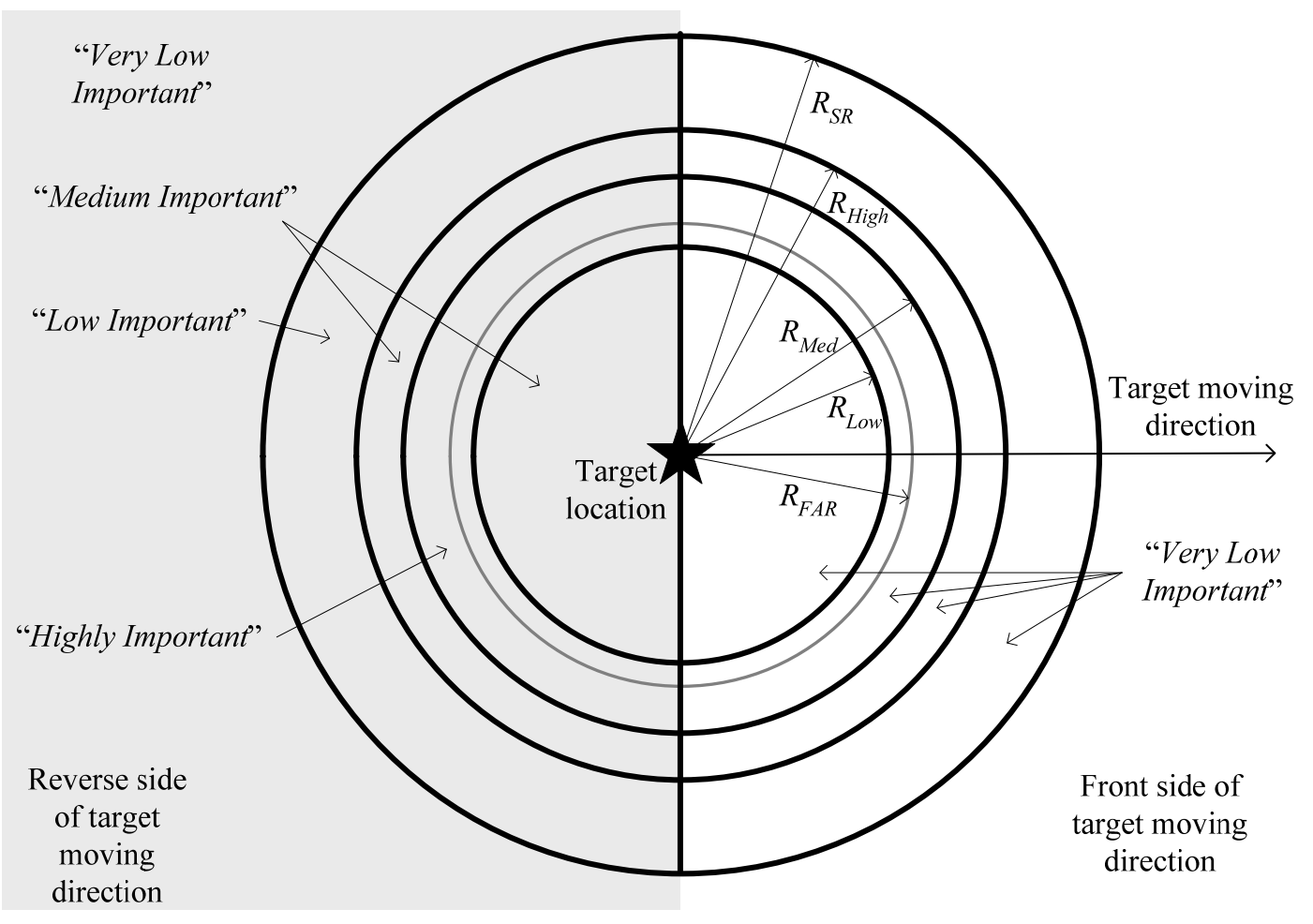

Figure 4.8 Sensor Node Categorization

The distances $R_{S R}$ and $R_{F A R}$ denote the sensing range of the sensor nodes and the far field range threshold distance. $R_{\text {Low }}, R_{\text {Med }}$ and $R_{\text {High }}$ are predetermined distance values 
that define the regions with $R_{\text {Low }} \leq R_{F A R} \leq R_{\text {Med }}<R_{H i g h} \leq R_{S R}$. The values of $R_{\text {Low }}, R_{\text {Med }}$ and $R_{H i g h}$ will depend on the sensor node density, $R_{F A R}$ and $R_{S R}$.

The nodes that are located close to the far field boundary and in the reverse side of the target moving direction are the most likely to be selected for the localization sensor group (within next few time intervals) and are categorized as "Highly Important". The nodes that are very close to the target at the reverse side of the target moving direction are highly unlikely to be selected as members of the localization sensor group. However, they are highly likely to participate in the communication between two master nodes. Thus, they are placed in the "Medium Important" category. Since the nodes that are located in the front side of the target moving direction are undesirable for the localization sensor group, they are placed in the "Very Low Important" category. The other nodes are categorized as "Medium Important", "Low Important" or "Very Low Important" based on the distance from the target, with the intuition that closer the nodes are, higher the chance of them participating in the tracking process.

Above categorization assigns the node category based on the region the node is located. The regions are defined by the target location and moving direction. Therefore, it is not possible to achieve this categorization at each node independently in a distributed manner, as most of the nodes will not be aware of the target state estimation. Thus, the region each node belongs to is heuristically determined based on the received signal strength. Specifically, recently received acoustic signal strengths during the last few time intervals are stored in the application layer. Then, the change pattern of the received signal strengths is used to roughly estimate whether the target is approaching, leaving or staying stationary compared to the sensor node. In addition, the received acoustic signal 
strengths are used to roughly estimate the distance from node to the target. Using this information, the category each node belongs to is determined by the following algorithm.

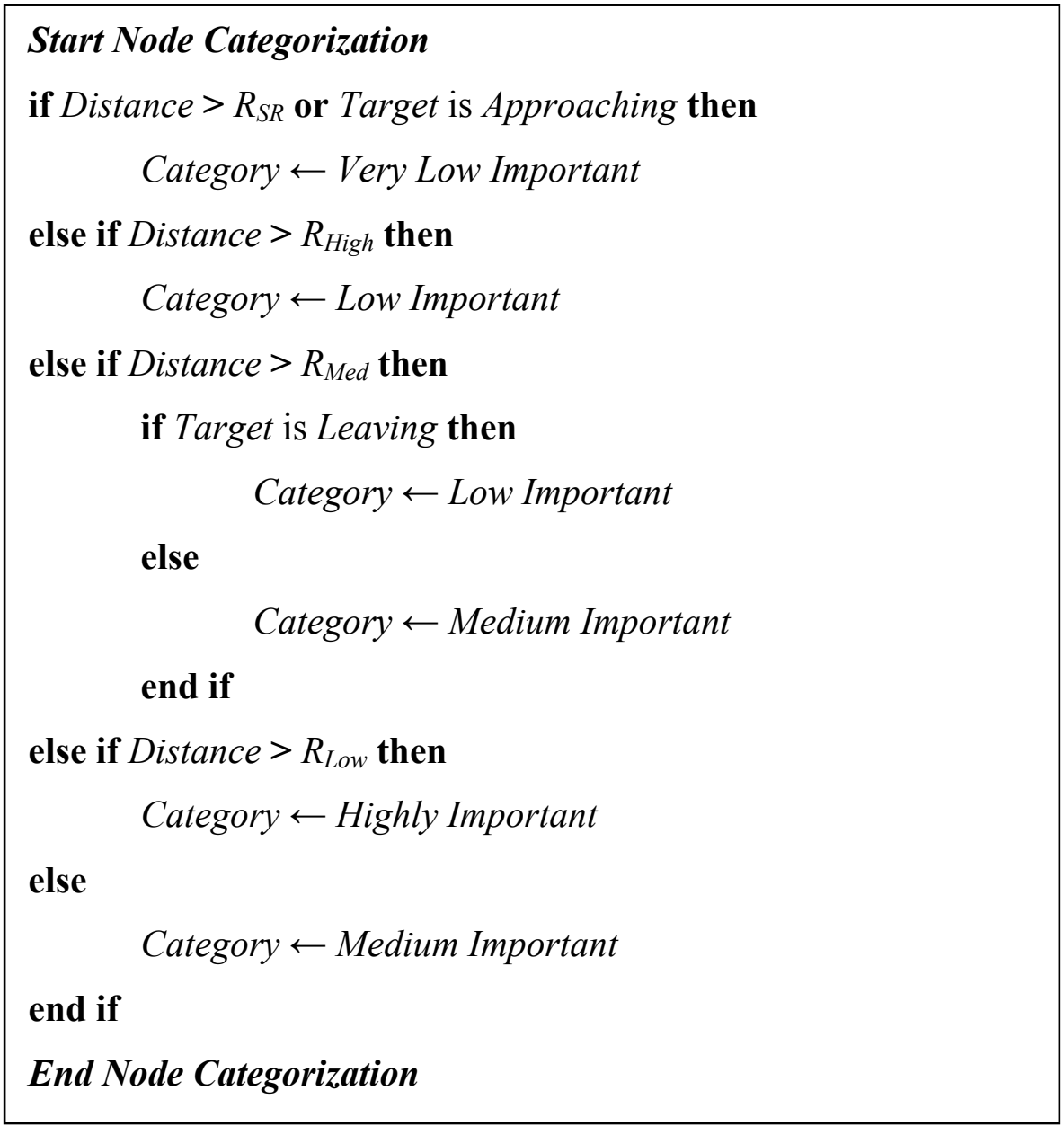

A coarse estimate of the total number of nodes within the one-hop neighborhood of the sensor node is obtained from the MAC layer to estimate the network condition. The traffic information of the network was not incorporated as the traffic pattern is highly correlated to the target tracking events. In addition, the remaining energy of the sensor node is also considered in adjusting the wakeup probability. With the sensor node classification and the neighboring node count, the wakeup probability $P_{a}$ is dynamically adjusted at each node using the following algorithm. 


$$
\begin{aligned}
& \text { Start Wakeup Probability Adjustment } \\
& \text { if Category is Highly Important then } \\
& \text { if Energy Left }<E_{V e r y L o w} \text { then } \\
& \qquad P_{a} \leftarrow \lambda\left[N C_{V H} / N N C\right] \\
& \text { else } \\
& \qquad P_{a} \leftarrow\left[N C_{V H} / N N C\right] \\
& \text { end if }
\end{aligned}
$$

else if Category is Medium Important then

if Energy Left $<E_{\text {Low }}$ then

$$
P_{a} \leftarrow \gamma\left[N C_{C o m m} / N N C\right]
$$

else

$$
P_{a} \leftarrow\left[N C_{C o m m} / N N C\right]
$$

end if

else if Category is Low Important then

if Energy Left $<E_{\text {Low }}$ then

$$
P_{a} \leftarrow \operatorname{Pr}_{\text {VeryLow }}
$$

else

$$
P_{a} \leftarrow \delta\left[N C_{C o m m} / N N C\right]
$$

\section{end if}

else

$$
P_{a} \leftarrow \operatorname{Pr}_{\text {VeryLow }}
$$

end if

if $P_{a}<P r_{\text {VeryLow }}$

$$
P_{a} \leftarrow \operatorname{Pr}_{\text {VeryLow }}
$$

else if $P_{a}>P r_{\text {VeryHigh }}$

$$
P_{a} \leftarrow P r_{\text {VeryHigh }}
$$

end if

End Wakeup Probability Adjustment 
Here, $N C_{V H}$ and $N C_{C o m m}$ represent the preset parameters that can be used to adjust the network connectivity level for high and medium important nodes. $N N C$ represents the estimated neighboring node count and $P r_{\text {VeryLow }}$ and $P r_{\text {VeryHigh }}$ are the two extreme probability limits. The energy limits $E_{\text {VeryLow }}$ and $E_{\text {Low }}$ can be decided beforehand depending on the energy consumption of the sensor nodes for sensing, communication and idle listing tasks. Moreover, the preset weight factors $\lambda, \gamma$, and $\delta(\lambda, \gamma, \delta \leq 1)$ can be used to adjust the energy usage balancing and the target tracking quality. The combination of $N C_{V H}, N C_{C o m m}$ and the $N N C$ is used to determine the required wakeup probability to maintain the desired level of node connectivity, which is decided by the application level classification of the node and the energy left in the node.

\subsection{Cross Layer Enhancements}

The anycast based message forwarding mechanism of the MAC protocol requires the location of the destination for successful message delivery (for unicasting). The message passing protocol (Section 4.4) supports this by including node locations in the required messages, so that when unicast communication between two nodes is required, the sender will always be aware of the location of the destination node. The exceptions for this are the message forwarding performed in the searches for the master nodes in initially forming the localization sensor group and updating the localization sensor group. The initial location estimation forwarding message (ILEF) forwarded by a unicast message along the far field boundary (see Sections 4.3.1.3 and 4.4.1.4) is not aware of a specific destination node. Since the forwarding is based on geometry, the sender is more interested in a node located close to the desired destination location. Similarly, the path of the localization sensor group update request messages (LSGUReq) is based on the 
geometry of moving direction of the target (see Sections 4.4.2 and 4.4.3). Thus, the sender (or a forwarding node) is more interested in selecting a node which is located close to the desired location.

In order to obtain a destination node for above scenarios, application layer has to depend on the MAC layer to obtain the locations of the neighboring nodes. However, this neighboring node information will not be updated in real-time. In addition, with the sleep-wakeup management incorporated, the selected destination node can be in sleep state as well. Moreover, the maintaining of neighboring node locations will induce additional load on the MAC protocol. Therefore, in order to eliminate these undesirable circumstances, a location based unicasting capability is incorporated to the anycast based message forwarding mechanism. The location based unicasting capability enables the application layer to send a packet to a node selected by the desired location. The destination node can be specified based on the desired geographical region, specified by the desired location and the radius of tolerance. This will eliminate the requirement for the MAC layer to maintain the neighboring node location information. In addition, as the destination is selected from the available nodes that are located in the desired geographical region, the message forwarding will not be affected by the sleep-wakeup management scheme. Figure 4.9 illustrates the forwarding scheme and the destination node selection of the location based unicasting scheme. 


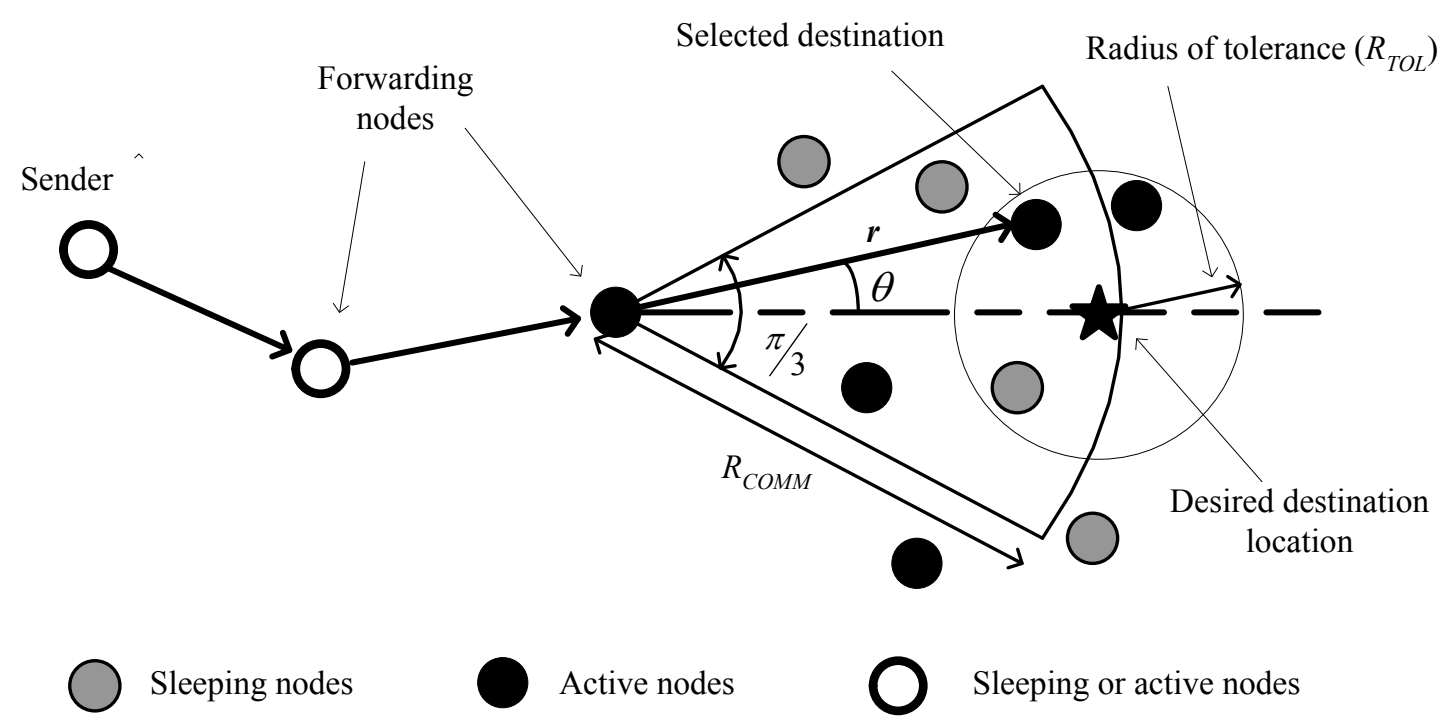

Figure 4.9 Location Based Unicasting Scheme

If there are unexpected delays in the network, overly delayed messages may not be useful for real-time tracking application. Therefore, forwarding these packets does not serve any purpose and will result in energy waste. In order to eliminate this waste, each message was assigned an expiration time. This expiration time is set by the application layer depending on the importance and timelines requirements of the message for successful tracking, and is included in the message header. The anycast based forwarding mechanism is modified to discard the messages that get delayed more than its expiration time. In addition, the data packets are assigned priorities by the application layer and the retry count of the packets is adjusted depending on the priority by the MAC layer in order to provide more robust communication path for the important application level packets. 


\section{CHAPTER V}

\section{PERFORMANCE EVALUATION}

The target tracking and energy conservation performance of the proposed energy-efficient distributed self-organization algorithm was evaluated using ns-2 [18] simulations. This chapter summarizes the performance evaluation of the algorithm. The first section presents an overview of the implementation. The simulation results and the conclusions drawn from the simulation results are discussed in the second section.

\subsection{The ns-2 Implementation}

This section provides the details of the implementation of the developed self-organization algorithm in ns-2 distributed event simulator. In the first part of the performance evaluation, the target tracking performance of the algorithm was evaluated. The default mobile node of the ns- 2 was used for this simulation, and a new application agent, namely the DSOAAgent was implemented. In the second part, effectiveness of the developed power management scheme was evaluated. For this, the routing and MAC layer protocols of the previous implementation were replaced by the MAC protocol developed by Wan et al. [16][17]. The MAC protocol includes an anycast based message forwarding scheme, and a sleep-wakeup management scheme. The cross layer wakeup probability adjustment scheme discussed in Section 4.5 and the cross layer enhancements discussed in Section 4.6 were implemented on top of this implementation.

\subsubsection{Overview of ns-2}

In the ns-2 software package, ns-2 stands for network simulator version 2. It is a discrete event simulator, which provides support for simulating network protocols over combinations of both wired and wireless networks. The software was initially developed 
based on REAL network simulator ([120]) in 1989. Currently it is maintained and enhanced through CONSER ([121]) and SAMAN ([122]) projects with collaborations from other researchers [18][123].

The ns-2 software package is distributed under open source software license and it supports simulating protocols of different layers of the OSI model, such as application layer, transport layer, network layer, data link layer and physical layer. Moreover, the ns-2 distribution contains a rich set of popular network protocols, and support functionalities, so that the researchers can easily conduct simulations and implement new protocols using these features. Due to these reasons, ns-2 is very popular among researchers for simulating network protocols.

The current wireless model of the ns-2 distribution is the mobility extension to ns-2, which was developed by Monarch project [123] of Carnegie Mellon University (now at Rice University) [124]. Mobile node is the major component of the ns-2 wireless model. The mobile node consists of following network components [124].

- Application agent (a traffic source, sink or a combination of both)

- Routing agent (routing protocols such as DSDV, DSR, TORA, and AODV)

- $\quad$ Link layer (LL)

- Address resolution protocol (ARP) module (connected to link layer)

- Interface priority queue (IFq)

- MAC Layer (several IEEE 802.11 MAC protocol versions available)

- Network interface (netIF)

The radio preparation module and the antenna module are attached to the network interface layer of the mobile node. Several propagation models, namely free space model, 
two-ray ground reflection model and shadowing model are implemented in the ns-2 distribution. The network interface connects the MAC layer and the channel. Within the mobile node, the incoming and outgoing traffic are filtered and directed to appropriate agent (application/routing) using the address and port classifiers. To support energy usage simulations, an energy model implementation is available for mobile nodes. The energy model has the parameters for the power consumption at transmitting, receiving and idling states, and the initial energy of the mobile node [124].

\subsubsection{Implementation of Self-Organization Algorithm in ns-2}

To evaluate the target tracking performance, the developed self-organization algorithm was implemented in ns-2 as a new application protocol. Specifically, the message passing protocol discussed in Section 4.4 was implemented in a new application agent (DSOAAgent) in ns-2.

In order to perform the searches for the two master nodes, the implementation of the application agent required information about the neighboring nodes. It was assumed that the networking layer (routing layer or MAC layer) provided an interface to obtain the required information. In this part of the implementation, the sleep-wakeup management scheme was not implemented. Greedy Perimeter Stateless Routing (GPSR) [125] protocol was used as the routing agent and the GPSR implementation for ns-2 provided by Karp (available at [126]) was used. The interface to provide the neighboring node information to the application layer was added to the GPSR implementation. For all other components of the mobile node except for the application and routing agent, the implementations available in the ns-2 distribution were used. The following figure 
illustrates the architecture of the mobile node with the protocols used for the simulation. The newly implemented and modified components are shaded in the figure.

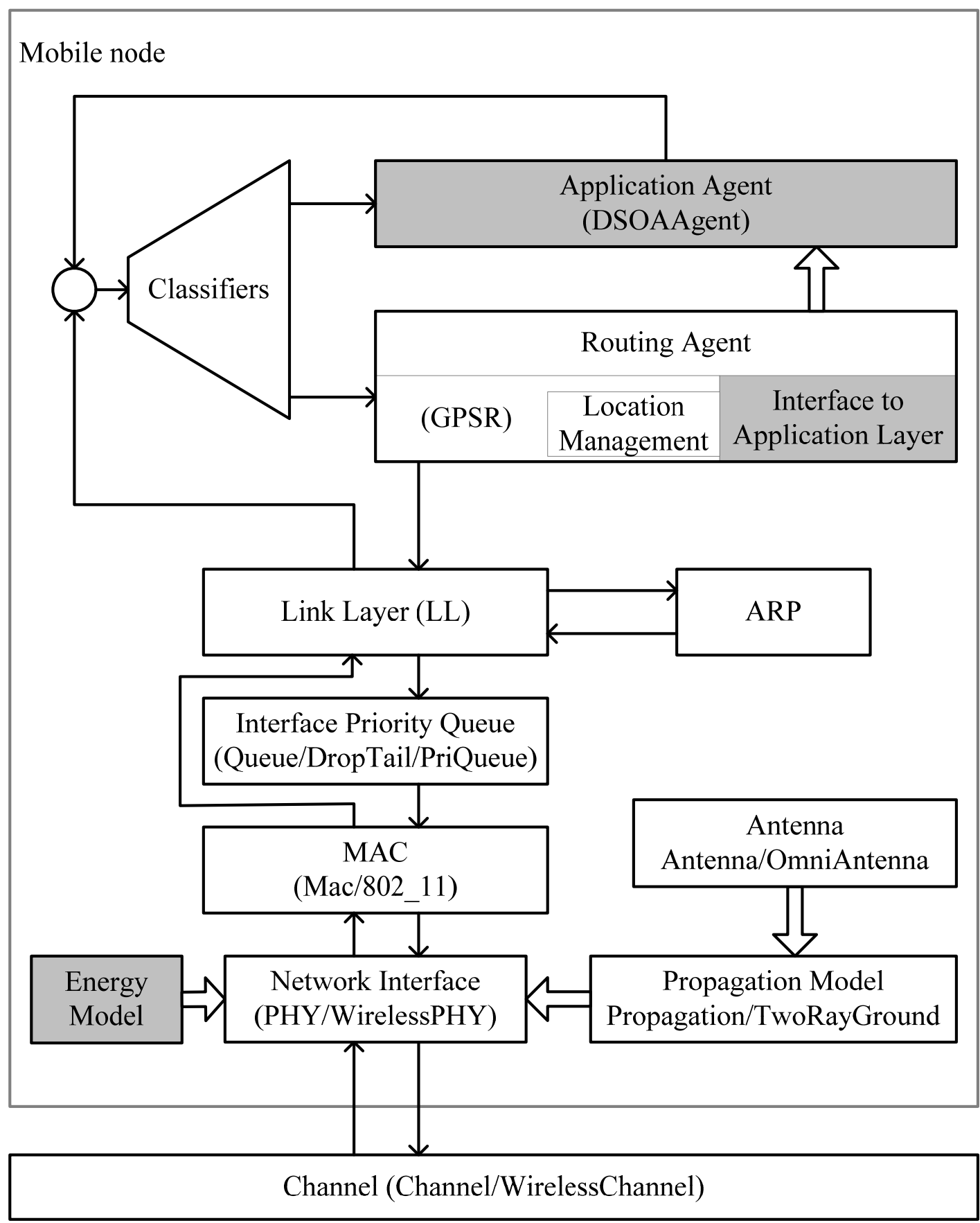

$\longrightarrow$ Packet flow

$\Longrightarrow$ Association between components

Figure 5.1 Architecture of the Mobile Node Used for the Simulation 


\subsubsection{Implementation of the DSOA Application Agent}

The proposed self-organization algorithm was implemented in an application agent named "Distributed Self Organization Algorithm" agent (DSOAAgent). An overview of the class diagram for the developed DSOAAgent class is shown below.

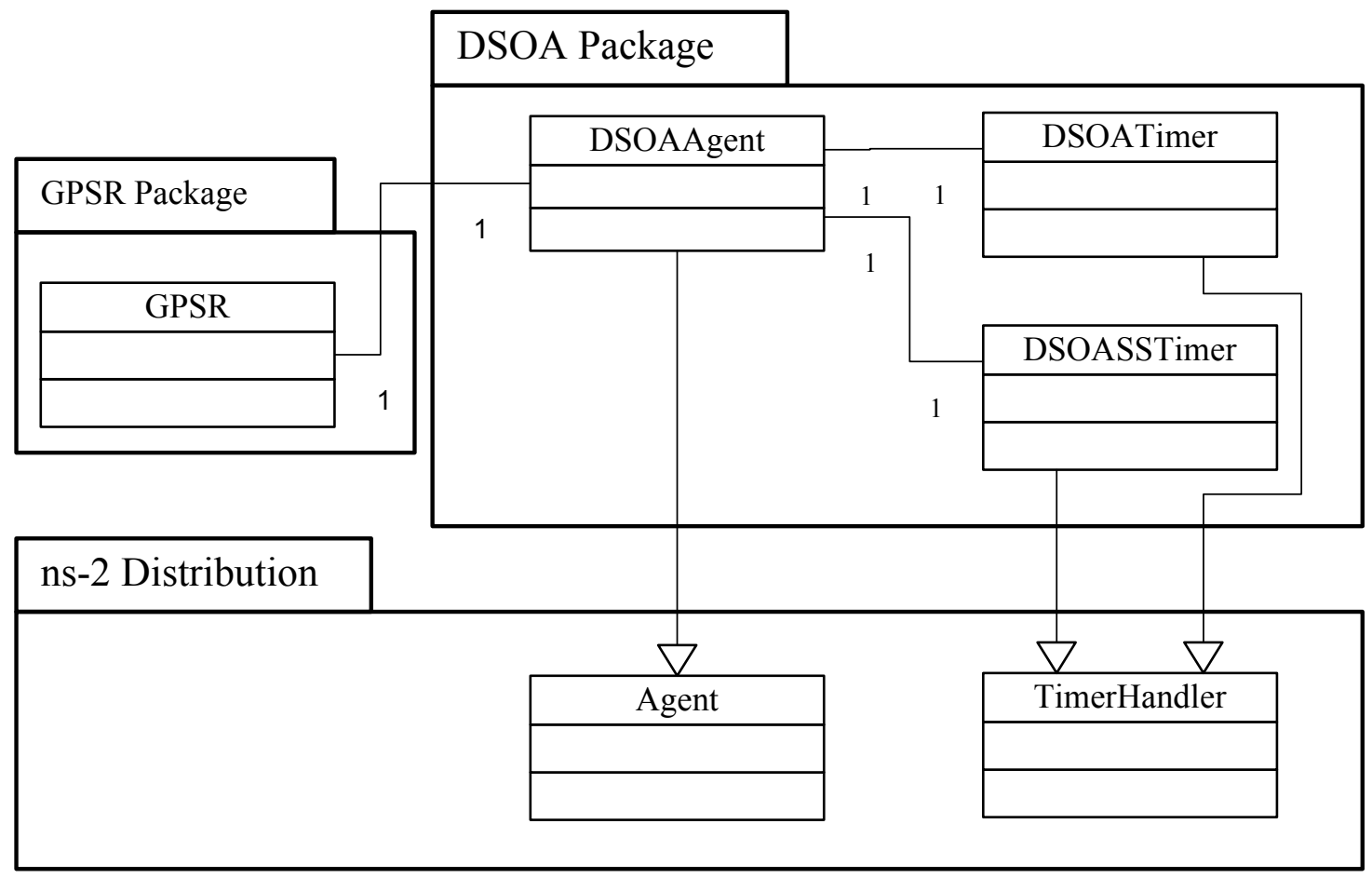

Figure 5.2 Overview of the Class Diagram for DSOA Application Agent Implementation

The DSOAAgent class was extended from the Agent class implementation provided in the ns- 2 implementation. Thus, all the interactions with the other components and packet flowing (shown in Figure 5.1) were handled through the functionalities of the Agent class. The developed DSOAAgent class contained the implementation of the message passing protocol developed in Section 4.4 and the Kalman filter functionalities for target tracking. In addition to the contents of the messages specified in Section 4.4, a header section (DSOA header) was assigned to the messages. This header contained the message type and a unique message ID. 
In addition, the DSOAAgent class contained the functionality required for the interacting with routing agent, simulating the acoustic signal receiving, and recording the simulation results. The implementation used two timers, namely DSOATimer and DSOASSTimer. Both of the timers were extended from the TimeHandler class of the ns-2 distribution. The DSOATimer was used for the handling of backoff timers required for the message passing protocol. The second timer was employed to simulate the receiving of the acoustic signal. An association with the GPSR agent was maintained at the application agent to obtain the neighboring node information from the GPSR agent.

\subsubsection{Modification of GPSR to Implement Interface for the Application Agent}

The GPSR implementation for ns-2 provided by Karp (available at [126]) was slightly modified by adding the functions required to provide neighboring node information to the application agent. The information was already available within the GPSR agent and the following two functions were added to the GPSR agent, so that application agent can access them.

- int GPSR_Agent::getNeighborNodeCount()

- int GPSR_Agent::getNeighborNode(int nodeIndex, ... )

The first function provided the number of nodes in the one-hop neighborhood of the node and the second function provided access to the details of a neighboring node.

\subsubsection{Modifications to the ns-2 Energy Model}

In this part of the simulation, only the application level energy considerations were implemented. Specifically, the energy considerations were incorporated to the node selection process. However, the sleep-wakeup management scheme was not incorporated. Thus, only the application level energy usage was considered in the energy saving 
analysis. In order to measure the effectiveness of the application level energy considerations, the energy model of the ns-2 mobile node was modified to consider only the energy consumed by the application level packets. The energy consumed by nodes in sending, receiving and relaying the application layer packets were considered. However, the communication overhead and the idle power consumption were neglected.

\subsubsection{Incorporating Energy Conservation}

In the second part of the simulation, the sleep-wakeup management scheme was incorporated to the algorithm. Thus, the developed DSOAAgent was integrated with the communication stack developed by Wan [17]. The protocol stack consists of implementation of four common services, namely; location management, coarse time synchronization, anycast data forwarding and energy management. Here, the routing agent was replaced by the "Dumb Routing" agent and the routing tasks were handled by the anycast based data forwarding scheme. The "Dumb Routing" agent acts as the interface between the application layer and the link layer. The location management provided the node location information for the anycast based data forwarding scheme. The Energy management coordinated the energy conservation through sleep-wakeup management scheme. Interactions with the application layer, anycast forwarding and coarse synchronization were required to determine the node state transitions and to dynamically adjust the wakeup probability. In addition, the node state transitions were performed through the energy model. The coarse synchronization handled the time synchronization and exchange of node information between one-hop neighbors. The architecture of the mobile node with the new communication stack proposed by Wan [17] is shown in Figure 5.3. The components with modifications are highlighted with shading. 


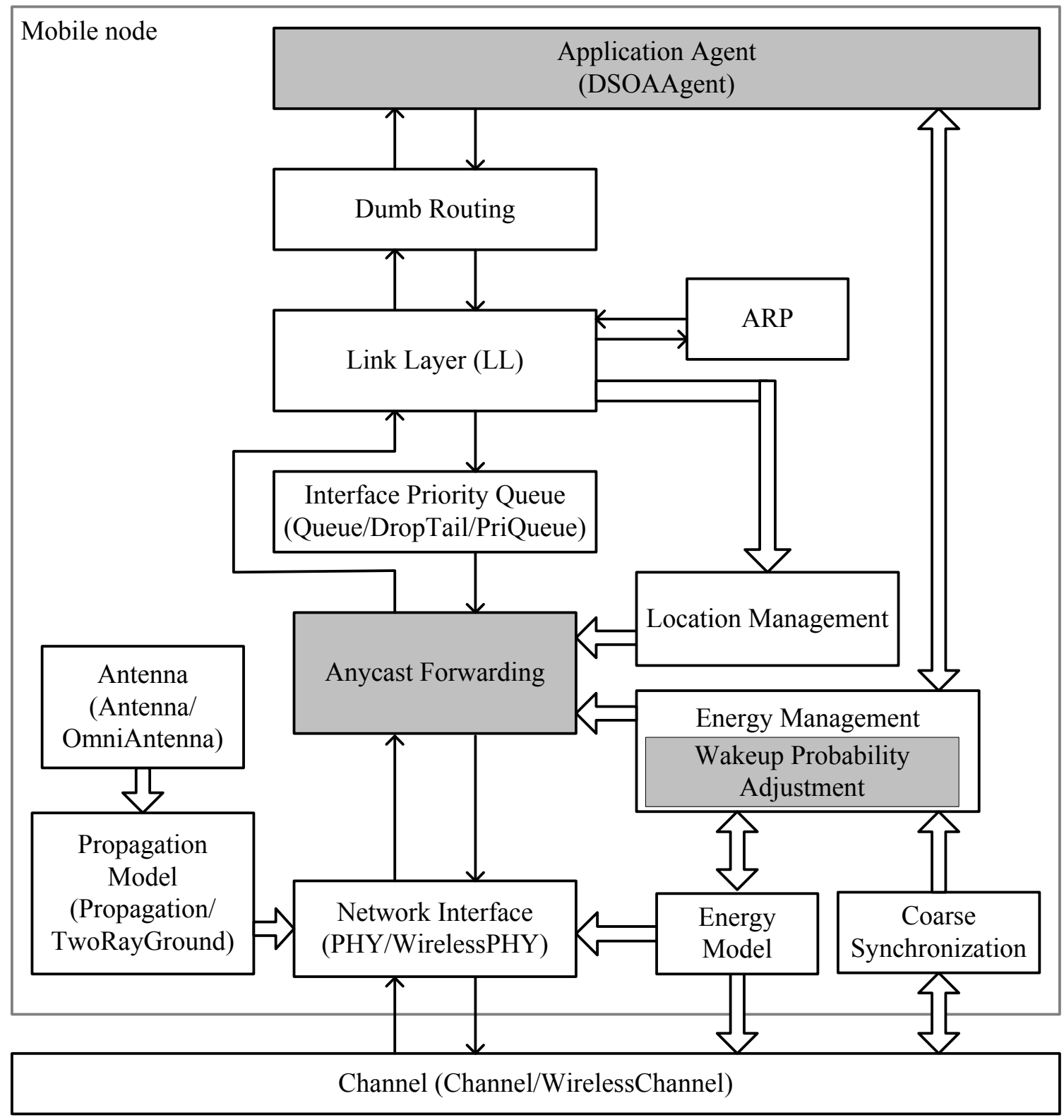

$\longrightarrow$ Packet flow

Association between components

Figure 5.3 Architecture of the Mobile Node after Integrating with the Communication Stack

\subsubsection{Wakeup Probability Adjustment Modification}

The wakeup probability adjustment functionality of the energy management module was modified to implement the developed cross layer wakeup probability adjustment algorithm discussed in Section 4.5.3. The event information required for the 
wakeup probability adjustment was obtained from the association through the application layer. The coarse synchronization module was responsible for providing the neighboring node information required for wakeup probability adjustment.

\subsubsection{Implementation of the Cross Layer Enhancements}

The cross layer enhancements discussed in Section 4.6 were incorporated into the anycast based data forwarding scheme of the MAC protocol. Thus, the anycast forwarding module of the communication stack was modified to implement these modifications. In implementing the location based unicasting, the forwarding scheme was updated and a new data packet type, namely location based unicasting packets, was introduced. The anycast based forwarding scheme handled this new type of data packets in a slightly different manner compared to regular packets. In forwarding the standard unicast packets, the destination node was fixed and the exact location of the destination node was known. Thus, the forwarding stopped when the packet reached the desired destination node, located at a known location. When forwarding location based unicasting packets, the exact destination node was unknown. However, the desired location of the destination was known. In this scheme, the forwarding terminated when the packet reached a node that was located in the desired region specified by the source node. The desired region was a circle centered at the desired destination location and was specified by the desired destination location, and the radius of tolerance. Except for the termination, all the other steps in the forwarding scheme were similar to the anycast based forwarding.

In addition, the message forwarding scheme was modified to include additional condition before forwarding the packet to check for the expiration time of the packet. If 
the expiration time was specified by the application layer and had elapsed, the message was discarded by the forwarding scheme, rather than forwarding it. Moreover, the retry count of the forwarding scheme was modified based on priority of the packet, which was assigned by the application agent.

\subsubsection{Implementation of Tracking Using Four Closest Nodes Group}

In addition to the proposed self-organization algorithm, a tracking algorithm that used the four closest nodes to track the target was implemented for the target tracking performance comparison. In this implementation, the four sensor nodes closest to the target, which were located in the far field were selected to perform the target localization. The Kalman filtering was applied to perform the target state estimation and prediction. This sensor group was updated in every other sampling interval to match the predicted target locations. The selection of the four closest nodes was performed by a search process carried out along the boundary of the far field.

\subsection{Simulation Results}

This section provides a discussion on the performance of the developed energy-efficient distributed self-organization algorithm. The simulation results obtained using the implementation of the algorithm in ns-2 and the conclusions from the obtained results are presented in this section. Specifically, the target tracking quality and the energy conservation characteristics of the developed algorithm are discussed in this section. In addition, the effect of sensor node density on tracking performance and the effectiveness of the developed energy aware node selection criterion in selecting the near-optimal sensor node group are evaluated. The simulation results confirm the 
effectiveness of the developed algorithm for target tracking using wireless acoustic sensor networks.

\subsubsection{Target Tracking Performance}

The target tracking quality of the developed self-organization algorithm was evaluated using the DSOAAgent implemented on the ns-2 distributed event simulator. For this part of the simulation, the sleep-wakeup energy management scheme was not used. In addition, the application level energy considerations were not used in this simulation. The simulation settings and the results obtained are discussed next.

\subsubsection{Simulation Setup}

In this simulation, tracking of a target moving inside a $1000 \mathrm{~m}$ by $300 \mathrm{~m}$ sensor field was simulated. 3000 sensors were randomly deployed in the sensor field in a uniform manner. The target was set to move at a speed of $10 \mathrm{~m} / \mathrm{s}$ along $x$ direction on a path that was $1000 \mathrm{~m}$ long. The sensing range of the sensor nodes was set to 300 meters. The communication range of the sensor nodes was set to 30 meters. The acoustic signal propagation process from the source to sensors was simulated by adding a random noise to the actual TDOA value. Specifically, the TDOA estimation between two sensor nodes was generated from the true TDOA value by adding random noise, which was uniformly distributed between $3.5 \mathrm{~ms}$ and $-3.5 \mathrm{~ms}$. The important parameter values of the message passing protocol that were used for the simulation are listed in the following table (A brief description of the parameters is provided here. The details of the parameters are available in Section 4.2 thru Section 4.4). 
Table 5.1 Parameter Values of the Message Passing Protocol for Target Tracking Performance Evaluation

\begin{tabular}{|c|c|c|}
\hline Parameter & Description & Value \\
\hline \multicolumn{3}{|c|}{ General Parameters } \\
\hline$L_{T H}$ & Maximum allowable sensor separation distance. & $30 \mathrm{~m}$ \\
\hline$R_{F A R}$ & Threshold distance to satisfy the far field assumption. & $100 \mathrm{~m}$ \\
\hline $\mathrm{C}$ & Speed of acoustic wave propagation in air. & $340 \mathrm{~m} / \mathrm{s}$ \\
\hline$T_{s}$ & Sampling interval. & $1 \mathrm{~s}$ \\
\hline$N_{I T}$ & $\begin{array}{l}\text { Number of TDOA estimation samples gathered by } \\
\text { initial FCN groups before target location estimation. }\end{array}$ & 4 \\
\hline$\sigma_{D, C O N S T A N T}$ & Default value for TDOA estimation error variance. & $3.5 \mathrm{~ms}$ \\
\hline \multicolumn{3}{|c|}{ Cost Function Threshold Values } \\
\hline$C_{T H}$ & Threshold increase in bearing estimation. & 0.2 \\
\hline$C_{I G, T H}$ & $\begin{array}{l}\text { Threshold value of initial FCN group member } \\
\text { evaluation cost function used to make the decision on } \\
\text { sending IGJRep message. }\end{array}$ & 0.75 \\
\hline$C_{b e, e, T H}$ & $\begin{array}{l}\text { Threshold value of bearing estimation cost function } \\
\text { used to make the decision on sending SNRep message. }\end{array}$ & 0.053 \\
\hline \multicolumn{3}{|l|}{ Backoff Times } \\
\hline$T_{B O, M A X}$ & $\begin{array}{l}\text { Maximum backoff time before sending IGJRep } \\
\text { message. }\end{array}$ & $0.15 \mathrm{~s}$ \\
\hline$T_{I G S}$ & Backoff time before selecting the initial FCN groups. & $0.4 \mathrm{~s}$ \\
\hline$T_{I G E}$ & $\begin{array}{l}\text { Backoff time before estimating the target location using } \\
\text { TDOA estimations in initial FCN groups. }\end{array}$ & $0.4 \mathrm{~s}$ \\
\hline$T_{S N S}$ & Backoff time before selecting the slave node. & $0.15 \mathrm{~s}$ \\
\hline$T_{L S G S}$ & $\begin{array}{l}\text { Backoff time before selecting the two master nodes for } \\
\text { next LSG. }\end{array}$ & dynamic \\
\hline \multicolumn{3}{|c|}{ Time To Live (TTL) Values } \\
\hline$T T L_{I G H N}$ & Initial TTL value for IGHN Message. & 5 \\
\hline$T T L_{I G T E}$ & Initial TTL value for ILEF Message. & 20 \\
\hline$T T L_{L S G U}$ & Initial TTL value for LSGUReq Message. & 12 \\
\hline \multicolumn{3}{|c|}{ Weight Factors for Energy Consideration at Node Selection } \\
\hline$\alpha$ & Energy factor in master node selection process. & 1 \\
\hline$\beta$ & Energy factor in slave node selection. & 1 \\
\hline
\end{tabular}


In the above setting, the value for $T_{L S G S}$ was not specified. During the simulation, the $T_{L S G S}$ was dynamically calculated by the current master node of the master sensor pair using the following equation.

$$
T_{L S G S}=\left(\text { Next Tracking Time }-2.5 \times T_{S N S}\right)-\text { Current Time }
$$

$T_{L S G S}$ was selected in such a manner so that the formation of the next localization sensor group can be completed in time to perform the real-time target tracking with fixed sampling intervals. The setting of values for other backoff timers, $T_{B O, M A X}, T_{I G S}, T_{I G E}$, and $T_{S N S}$ require the knowledge about the per-hop communication delay of the sensor network and the sampling interval. With this information, the suitable values for the above backoff timers can be estimated through offline analysis.

The values for $L_{T H}, R_{F A R}$, and $N_{I T}$ were set based on the results of simulations conducted in preliminary analysis. Higher value for $N_{I T}$ will result in more stable initial LSG, at the cost of increased resource usage and higher initialization delay. Sampling interval can also be selected using offline analysis. If the sampling interval is too large, the target tracking quality will be lower. However, smaller sampling intervals will require higher communication and computing resources for successful operation. The default TDOA estimation error variance was set to $3.5 \mathrm{~ms}$ based on previous research results [4][20]. The threshold value $C_{T H}$, can be used to control the LSG update frequency. It can be determined through offline analysis based on the target tracking quality requirements and resource limitations. In our work, it was set to 0.2 based on the preliminary simulation results. $C_{I G, T H}$ was set to 0.75 times the optimal $C_{I G}$ value and $C_{b e, e, T H}$ was set to the value of $C_{b e, e}$ when the value of " $L \sin (B)$ " is 0.75 times the optimal " $L \sin (B)$ " value, which is $30 \mathrm{~m}$. 
The time to live (TTL) values of the algorithm were set offline. The values for $T T L_{I G T E}$ and $T T L_{L S G U}$ were set to the above values to ensure that the master node search messages will traverse the entire path along the far field boundary. TTLIGHN was set to 5 using the results from preliminary analysis to make sure that the head nodes will receive information about the neighboring head nodes with high probability. A higher $T T L_{I G H N}$ value will increase the chances of neighboring head node discovery. However, it will exponentially increase the communication requirements. The energy weighting factors $\alpha$ and $\beta$ can be used to adjust the optimality of the selected groups and the application level energy balancing. Both were set to 1 as the application level energy was not considered in the first simulation. However, these factors were set appropriately in the later parts of the performance evaluation.

In addition to these application level settings, there were some important parameter settings required for the protocol stack of the ns- 2 mobile node. The values assigned for those parameters are shown in the following table.

Table 5.2 Mobile Node Parameter Values for Target Tracking Performance Evaluation

\begin{tabular}{|c|c|c|}
\hline Parameter & Description & Value \\
\hline \multicolumn{3}{|c|}{$P H Y /$ Wireless $P H Y$} \\
\hline RXThresh_ & $\begin{array}{l}\text { Reception threshold. Defines the communication } \\
\text { range }(30 \mathrm{~m}) \text { of mobile node. }\end{array}$ & $0.213643 \mu \mathrm{W}$ \\
\hline CSThresh_ & $\begin{array}{l}\text { Carrier sense threshold. Defines the interference } \\
\text { range }(60 \mathrm{~m}) \text { of mobile node. }\end{array}$ & $0.0534106 \mu \mathrm{W}$ \\
\hline \multicolumn{3}{|l|}{ EnergyModel } \\
\hline energyModel & Energy model used for the simulation. & "none" \\
\hline
\end{tabular}

The target location estimated by the Kalman filter based tracking method was compared with the actual target location to generate tracking errors. A single movement of target from start to end of the route was a single simulation round. 
Target tracking was performed using the four closest nodes group for performance comparison. The sensor network parameters such as sensor node density and the sensor node locations, the ns-2 mobile node parameters and other general parameters (sensing range, communication range, far field range, etc.) were set to the exact same values as in the previous scenario. However, the parameters that were specific to the message passing protocol did not apply in this scenario.

\subsubsection{Results and Discussion}

Target tracking performance results obtained for the two algorithms using 20 simulations are summarized in the following figures. The time axis represents the elapsed time since the start of the simulation.

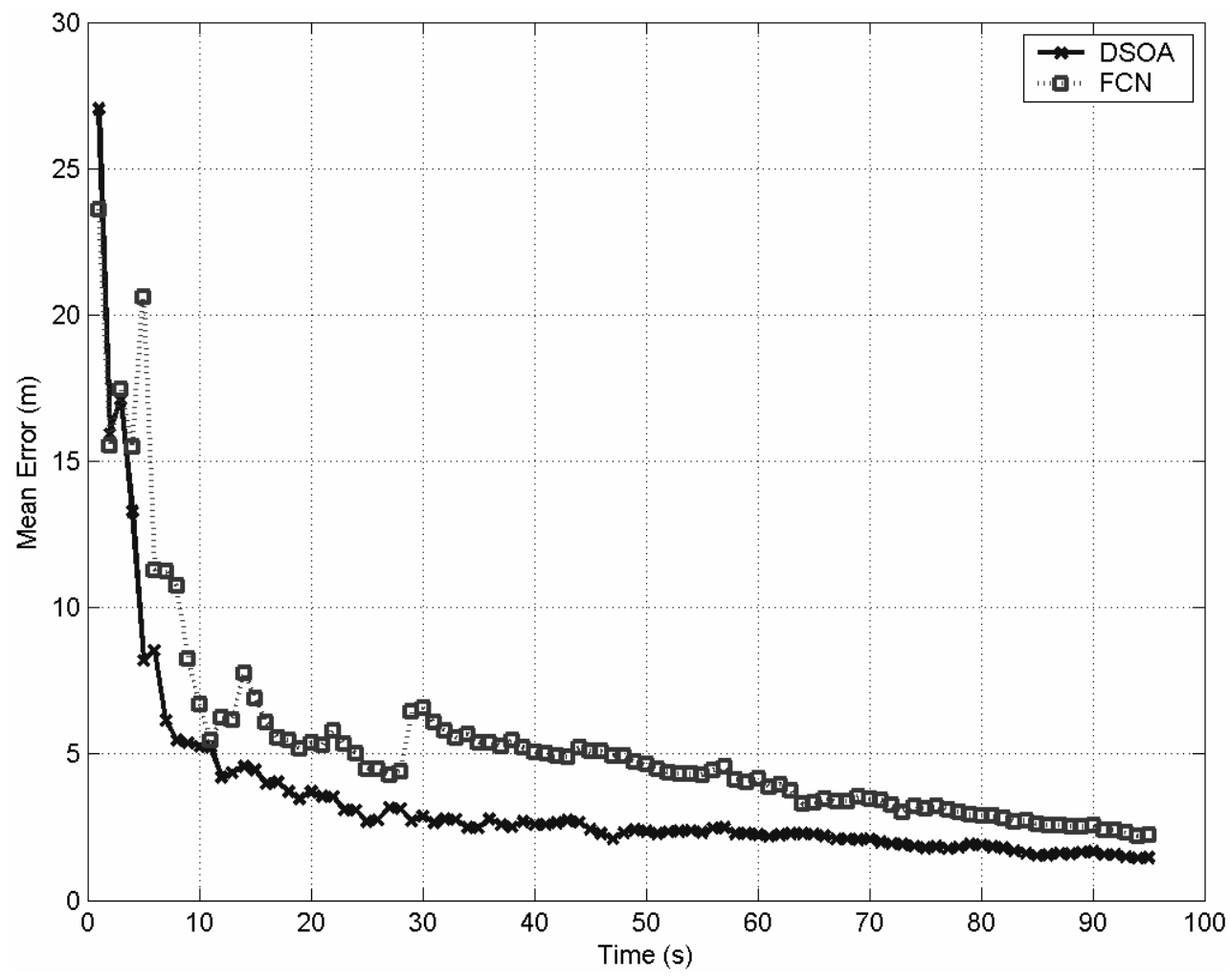

Figure 5.4 Mean Target Tracking Error in $x$ Direction for Two Algorithms 


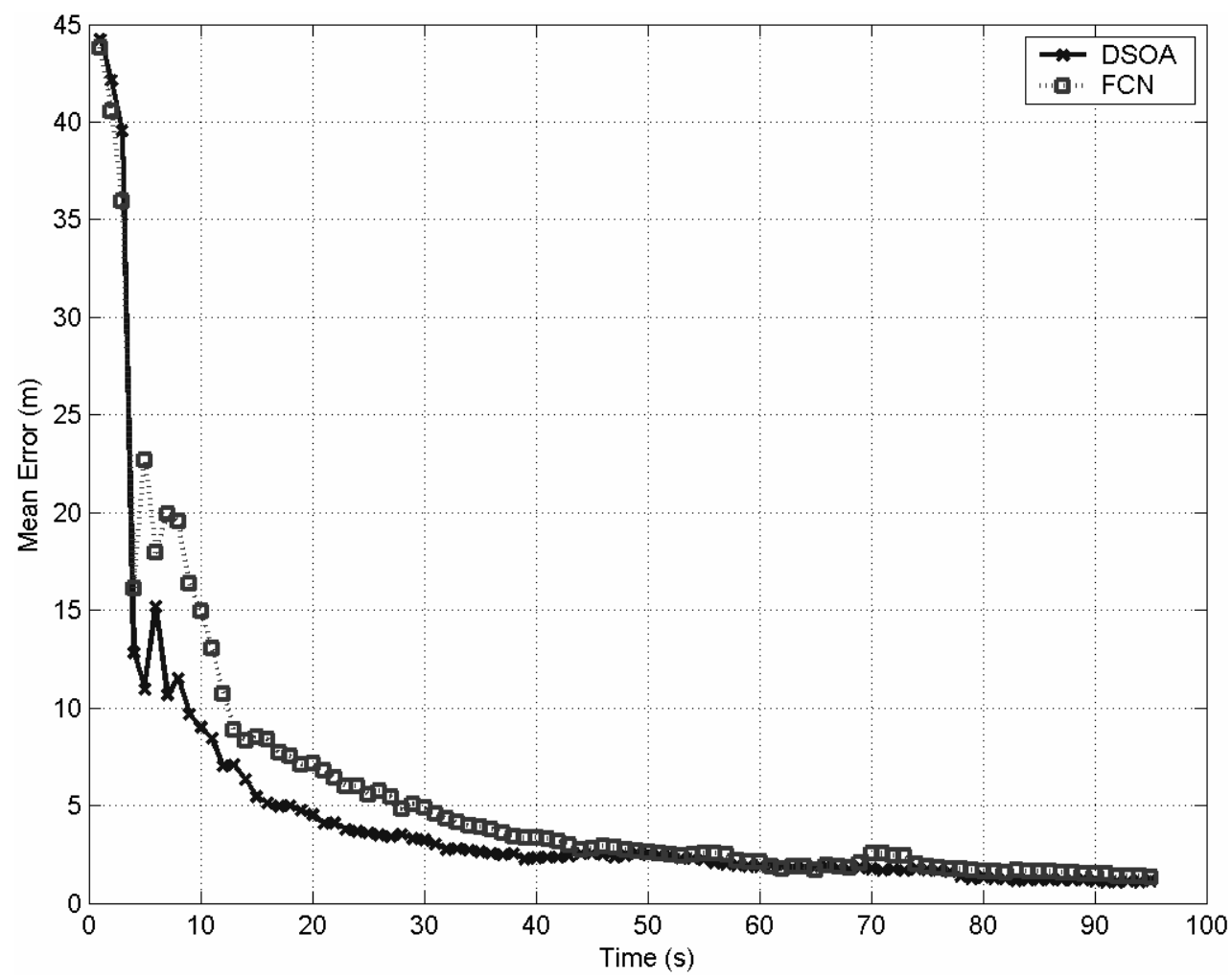

Figure 5.5 Mean Target Tracking Error in $y$ Direction for Two Algorithms

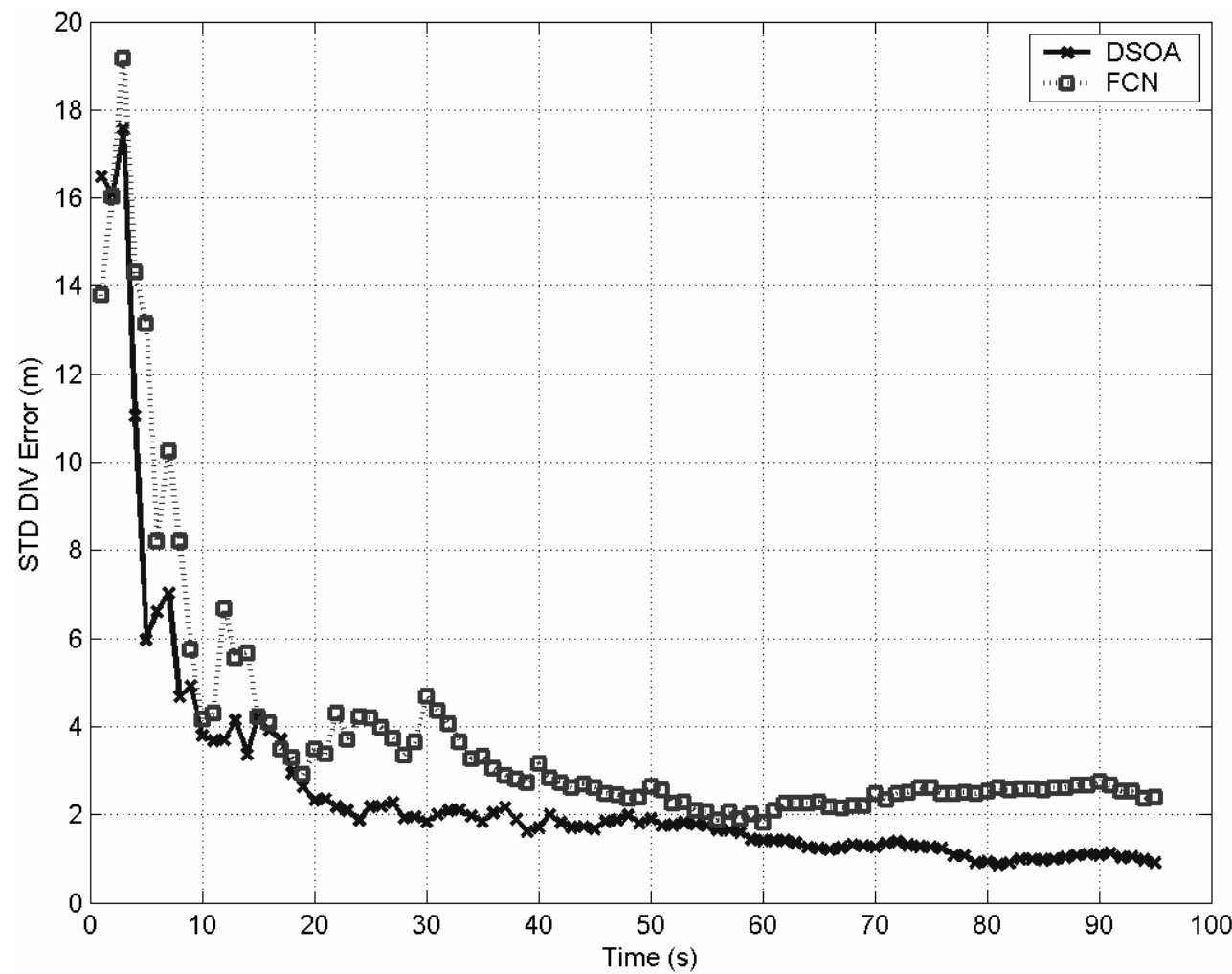

Figure 5.6 Standard Deviation of Target Tracking Error in $x$ Direction for Two Algorithms 


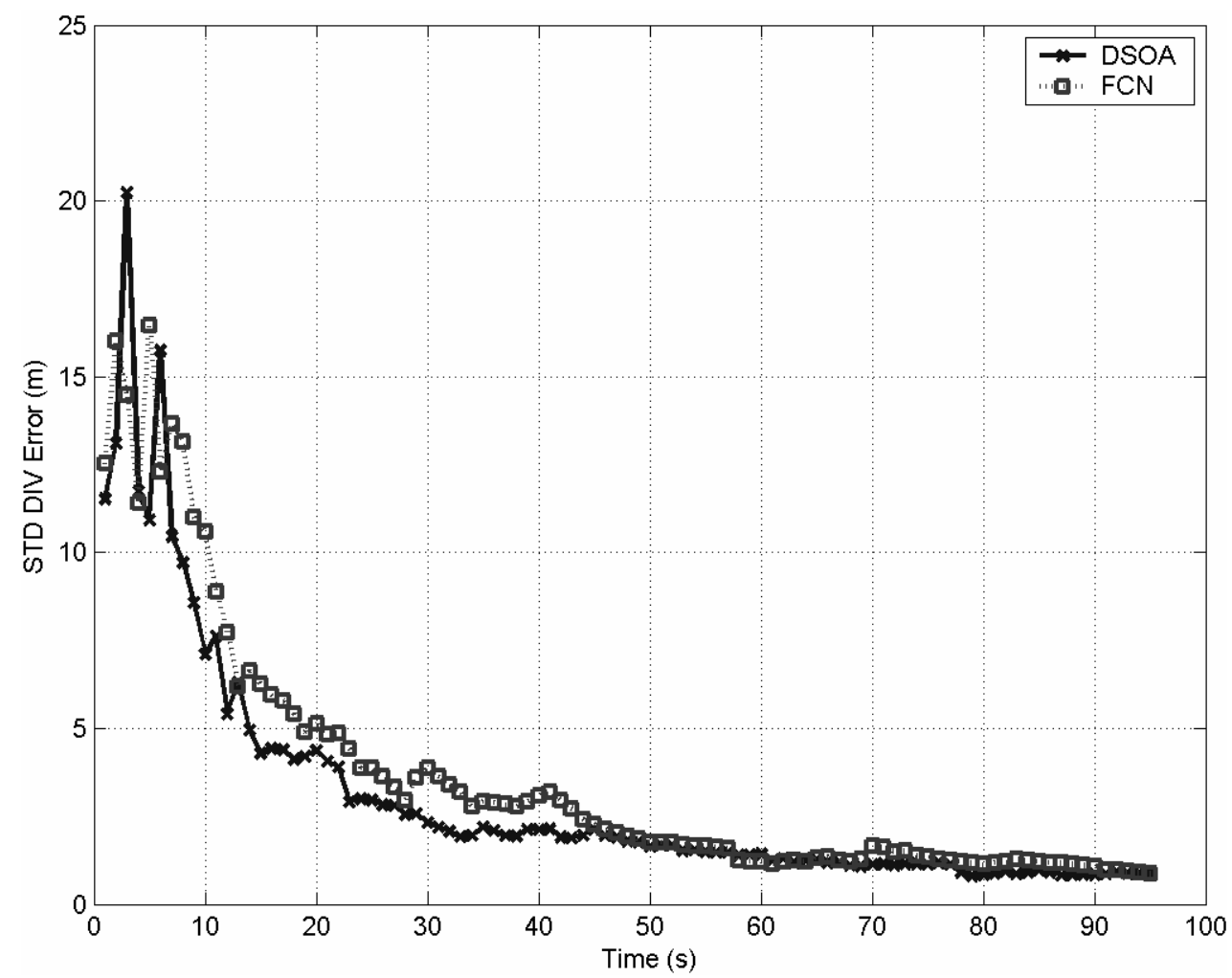

Figure 5.7 Standard Deviation of Target Tracking Error in $y$ Direction for Two Algorithms

It can be seen from the above figures that the mean and the standard deviation of the target tracking errors in both $x$ and $y$ directions were lower when the proposed self-organization algorithm was used for the target tracking. The average of the mean, and the standard deviation, of the target tracking errors for the two algorithms are shown in the following table.

Table 5.3 Summary of Tracking Performance Comparison for Two Algorithms (Average of the Mean and the Standard Deviation of Tracking Errors)

\begin{tabular}{|c|c|c|}
\hline Performance & DSOA & FCN \\
\hline Mean $-x$ & 3.4070 & 5.3162 \\
\hline Mean $-y$ & 4.4688 & 5.7355 \\
\hline STD $-x$ & 2.5690 & 3.8034 \\
\hline STD $-y$ & 3.0509 & 3.6357 \\
\hline
\end{tabular}


The summary of results shown above (Table 5.3) also confirms that the mean and the standard deviation of the tracking error were lower for the proposed self-organization algorithm. Therefore, it is evident from the above simulation results that the proposed self-organization algorithm outperforms the four closest nodes selection algorithm in dynamic target tracking performance. The large initial localization errors seen in the Figures 5.4-5.7 were caused by the measurements of the initial four closest nodes groups employed at the localization sensor group initialization stage of the self-organization algorithm. However, in the subsequent steps the combined effect of employing the localization sensor group and the Kalman filtering reduces the tracking errors to a satisfactory level.

\subsubsection{Effect of Application Level Energy Consideration}

The effect of application level energy consideration (at the node selection process) on the lifetime of the sensor network, and the target tracking performance, were evaluated using the same ns-2 implementation.

\subsubsection{Simulation Setup}

In this simulation, the size of the sensor field was reduced to $500 \mathrm{~m}$ by $300 \mathrm{~m}$. To maintain the same sensor node density the number of the sensor nodes was reduced to 1500. The sensor field size was decreased to reduce the time taken to complete the simulation. The path of the target was similar except for the shorter length of $500 \mathrm{~m}$. In this simulation, multiple targets that follow the same path enter the sensor filed one after another. The number of targets that can be tracked and the tracking quality for each target were recorded. 
All the parameters except for the energy related parameters were the same as in the previous simulation. The modified version of the ns-2 energy model discussed in Section 5.1.2.3 was used in the simulation. Table 5.4 shows the parameter values of the ns-2 energy model, which were used for this simulation.

Table 5.4 Energy Model Parameter Values for Analyzing the Effect of Application Level Energy Consideration

\begin{tabular}{|c|l|r|}
\hline \multicolumn{2}{|c|}{ Parameter } & \multicolumn{1}{c|}{ Description } \\
\hline EnergyModel & \multicolumn{2}{|c|}{ Value } \\
\hline energyModel & Energy model used for the simulation. & "Energy Model" \\
\hline rxPower & Power consumption in receiving state. & $282 \mathrm{~mW}$ \\
\hline txPower & Power consumption in transmission state. & $282 \mathrm{~mW}$ \\
\hline initialEnergy & Initial energy level assigned to the node. & $0.25 \mathrm{~J}$ \\
\hline
\end{tabular}

As it can be seen (from Table 5.4) a small initial energy value was set to further reduce the time required for simulation. In the first simulation, the application level energy considerations were not utilized. Thus, all the parameter values for the message passing protocol were unchanged. However, in the second simulation, the energy consideration was incorporated to the node selection process. Thus, the relevant parameter values were modified as shown in the following table.

Table 5.5 Parameter Value Changes of the Message Passing Protocol for Analyzing the Effect of Application Level Energy Consideration

\begin{tabular}{|c|l|r|}
\hline Parameter & \multicolumn{1}{|c|}{ Description } & Value \\
\hline \multicolumn{2}{|c|}{ Weight Factors for Energy Consideration at Node Selection } \\
\hline$\alpha$ & Energy factor in master node selection process. & 0.5 \\
\hline$\beta$ & Energy factor in slave node selection. & 0.33 \\
\hline
\end{tabular}



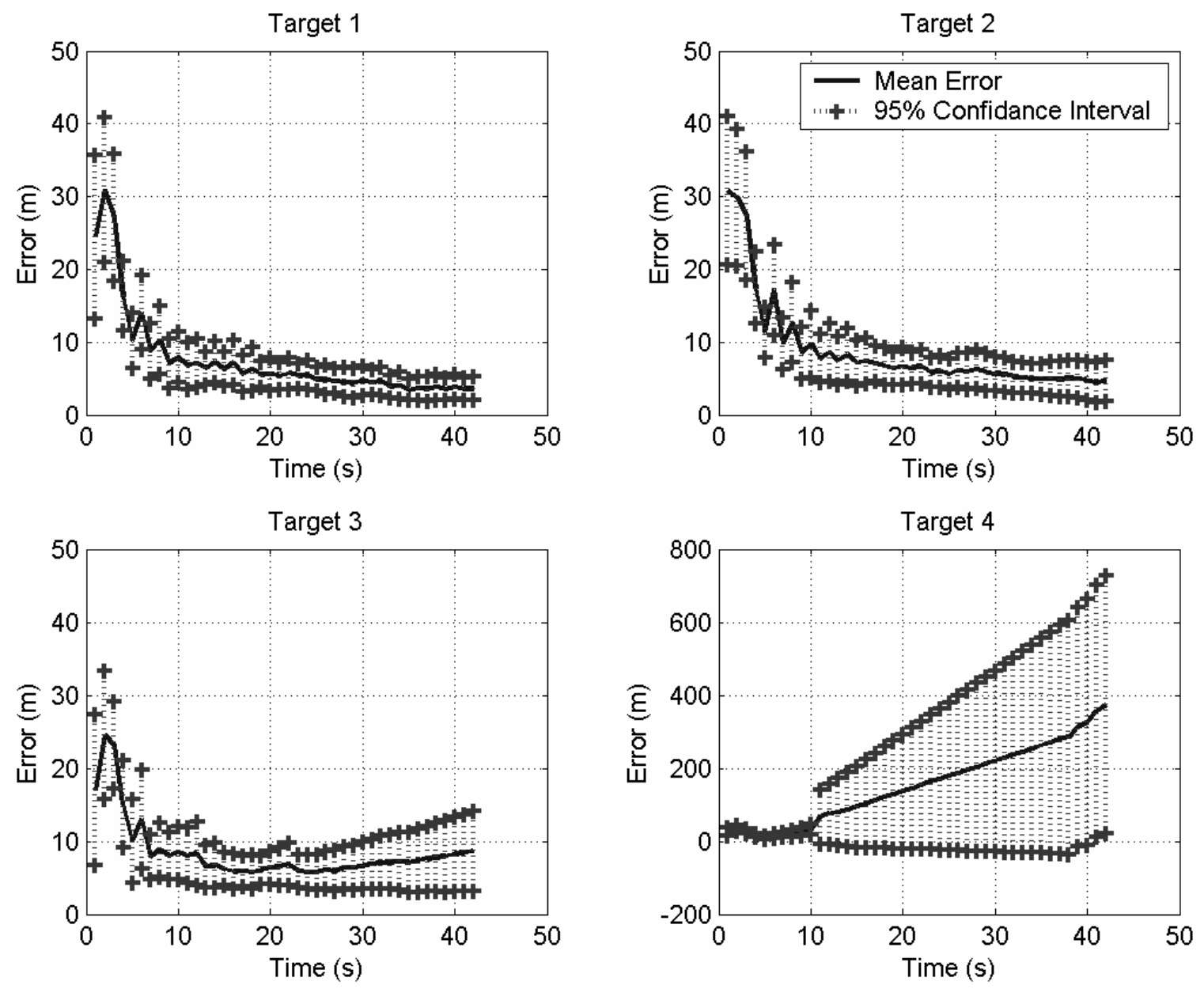

Figure 5.8 Target Tracking Performance in $x$ Direction without Application Level Energy Consideration

Target tracking performance in $x$ direction for the first simulation, where the application level energy considerations were not utilized, is shown in the above figure. In this scenario, four targets were tracked. However, it is evident from the above figure that the tracking of only the first two targets was successful. In the third target, the tracking quality degraded, resulting in poor tracking performance. The tracking errors were very high in the fourth target, which indicates that the sensor network was not able to track the fourth target. The high tracking errors were mainly caused by inability of the sensor 
network to support the communication required for the target tracking. This was caused by the dying out of sensor nodes after consuming the granted initial energy. Due to the lack of communication support, some measurements were missed and some localization sensor group update steps were not completed successfully in timely manner. The net effects of these two factors were reflected in the poor tracking quality observed during tracking third and forth targets. In case of the fourth target, the measurements were not available after the initial steps of tracking, which was the reason for huge tracking errors. Tracking performance in $y$ direction was similar and shown below.
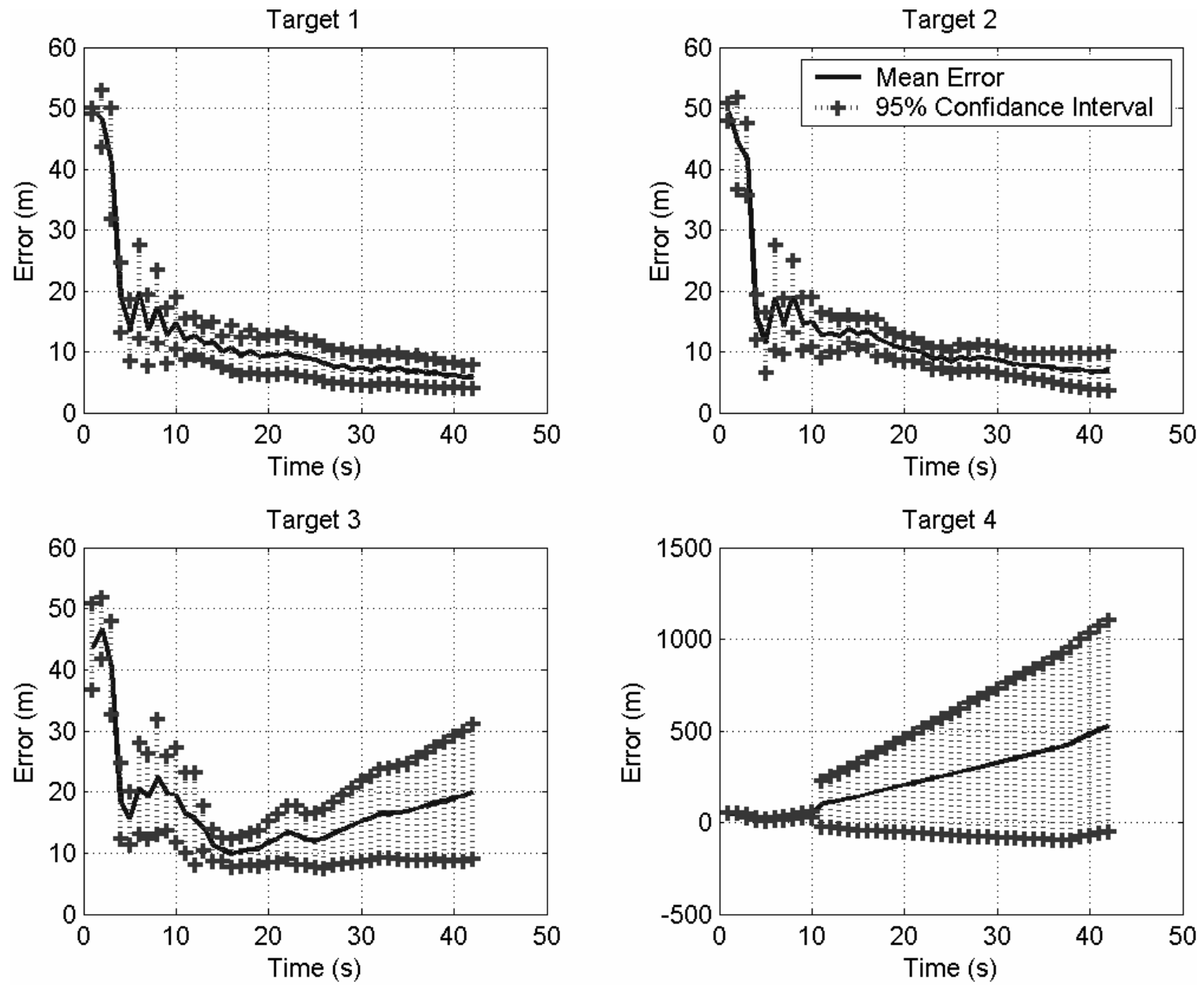

Figure 5.9 Target Tracking Performance in $y$ Direction without Application Level Energy Consideration 
Target 1

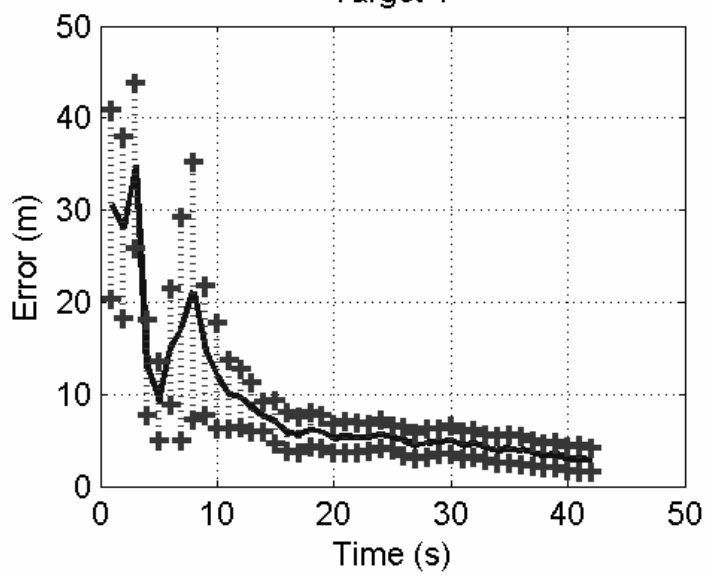

Target 3

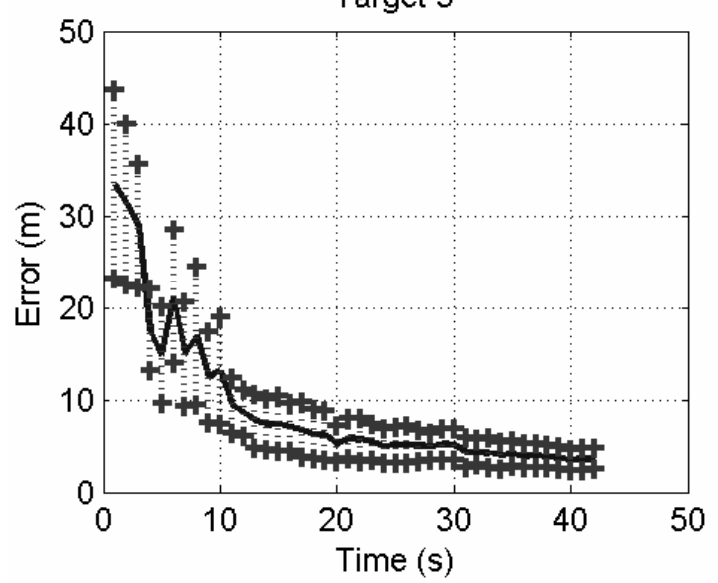

Target 5

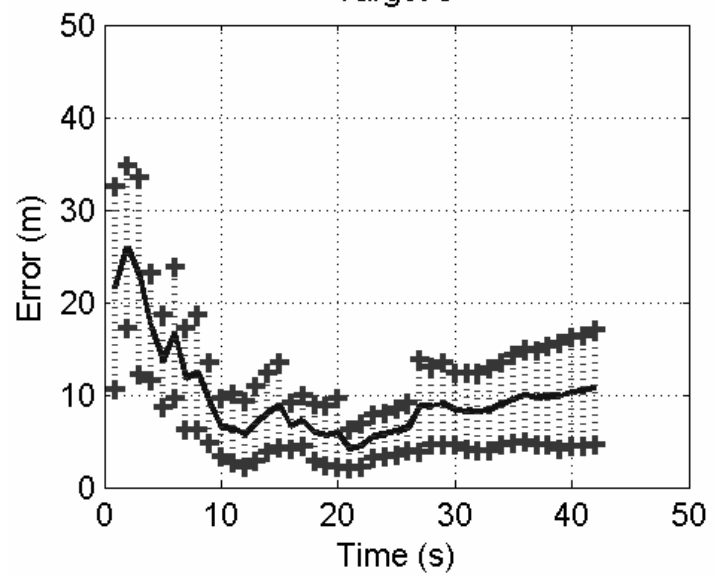

Target 2

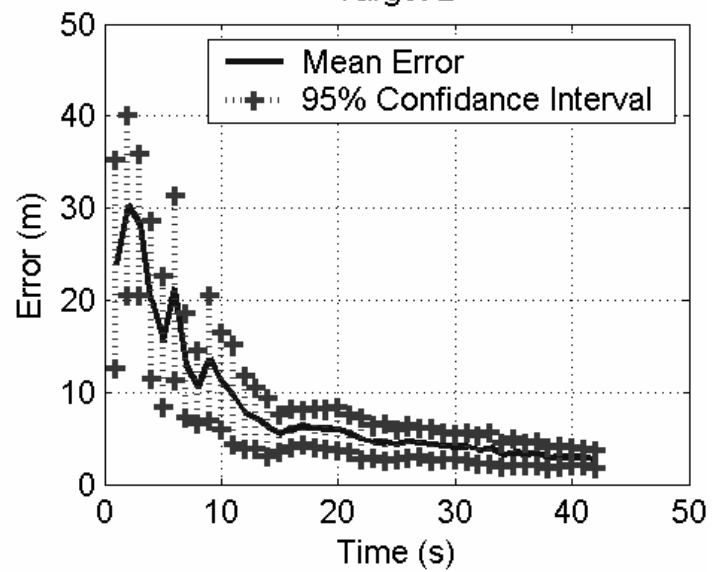

Target 4

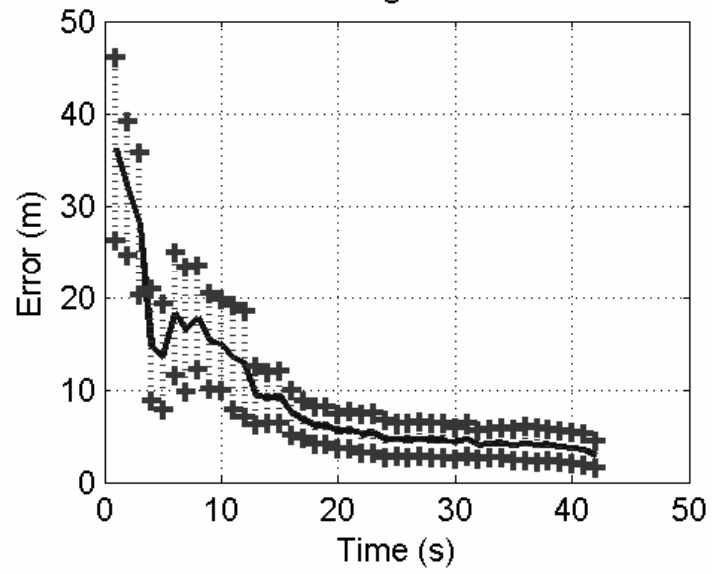

Figure 5.10 Target Tracking Performance in $x$ Direction with Application Level Energy Consideration 
Target 1
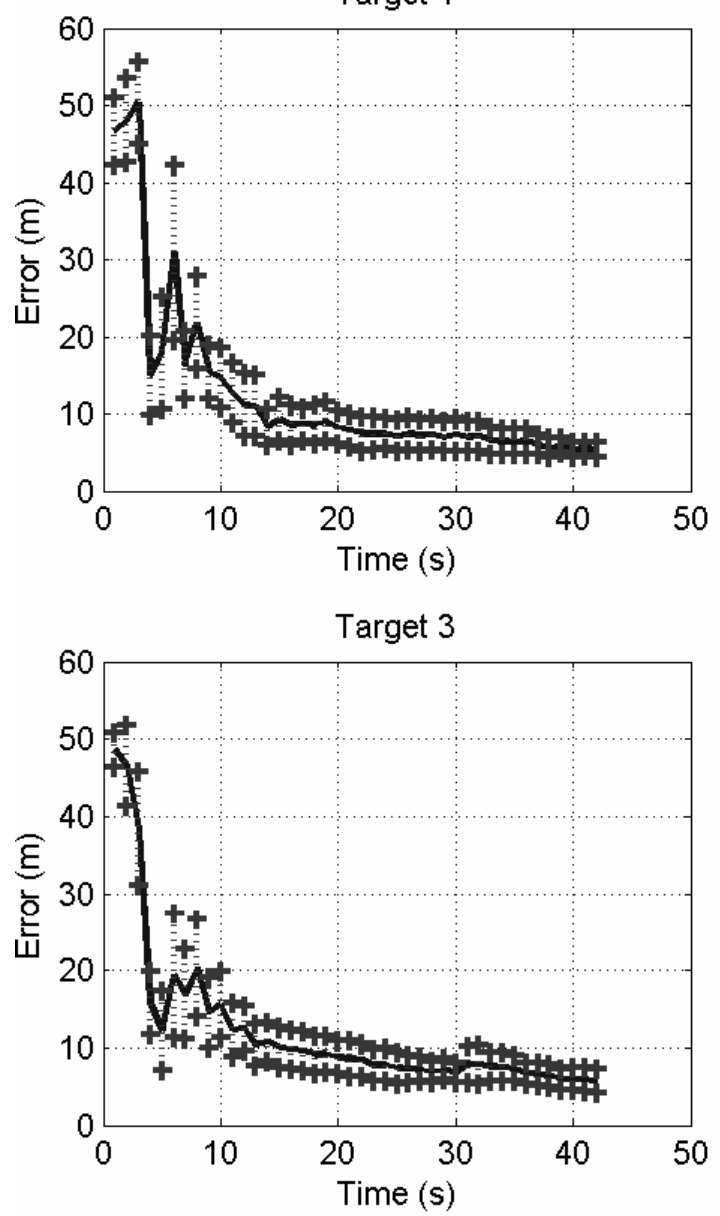

Target 5

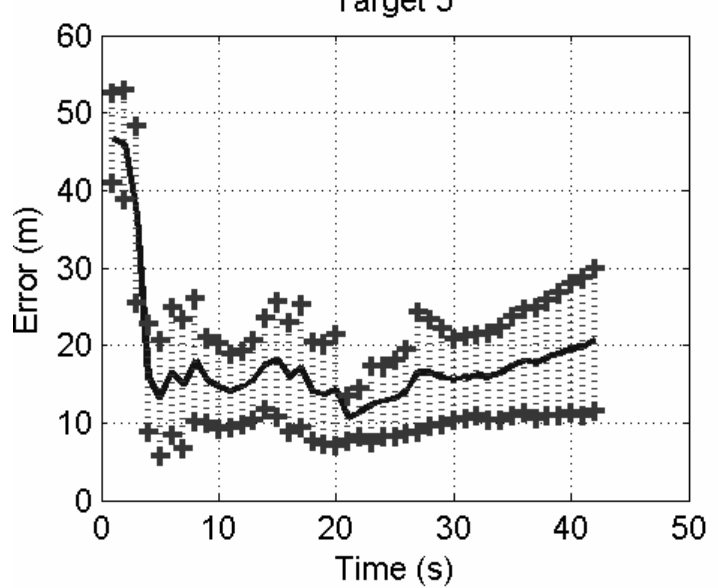

Target 2

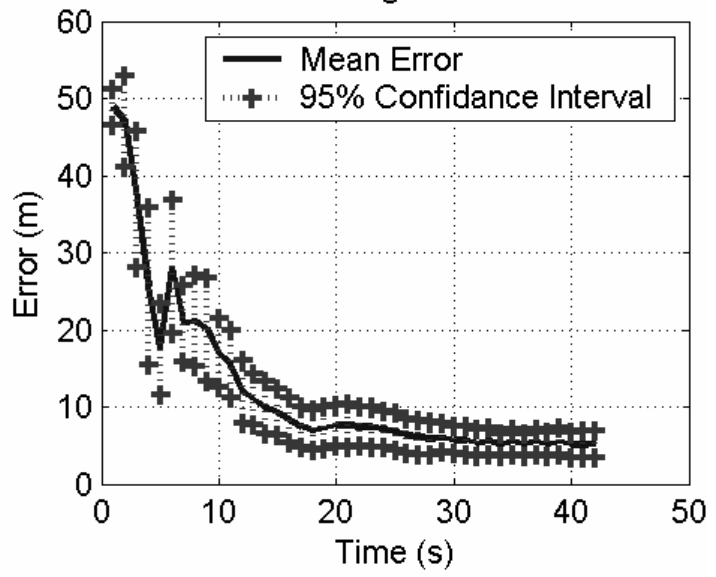

Target 4

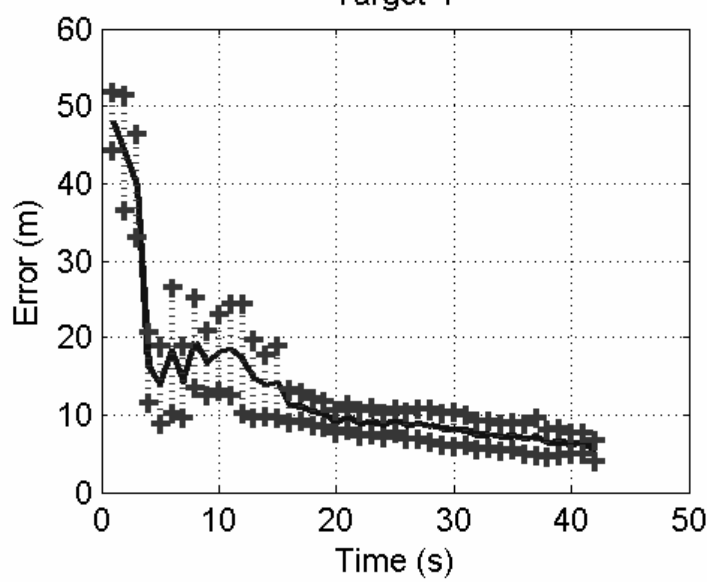

Figure 5.11 Target Tracking Performance in $y$ Direction with Application Level Energy Consideration

Target racking performance for the second simulation, where energy factors were considered in the node selection process, is shown in Figures 5.10 and 5.11. A total of 
five targets were tracked in this scenario. Moreover, the tracking quality of the first four targets was satisfactory. Thus, the incorporation of energy consideration to the node selection process has resulted in doubling the number of targets successfully tracked using the network. The target tracking errors were comparatively large in case of the fifth target, which indicates the inability of the sensor network to track further targets. The reason for the degradation in tracking quality of the fifth target was the same as the previous scenario.

These results confirm that incorporation of the energy consideration into the node selection process results in extended network lifetime. However, the incorporation of energy consideration into the node selection process would result in selecting a suboptimal localization sensor group. Under the assumption of high sensor node density, this group should be near-optimal and should perform very similar to the optimal localization sensor group. Therefore, the target tracking quality should be very similar for the two scenarios. The averaged mean and standard deviation (STD) of the tracking error magnitudes (of the successful target tracking runs) for the two parts of the simulation, shown in the following table demonstrate this observation.

Table 5.6 Summary of Tracking Performance Comparison for Application Level Energy Consideration (Average of the Mean and the Standard Deviation of Tracking Errors)

\begin{tabular}{|c|c|c|}
\hline Performance & Without considering energy & With considering energy \\
\hline Mean $-x$ & 8.1656 & 8.7174 \\
\hline Mean $-y$ & 12.6538 & 12.3999 \\
\hline STD $-x$ & 5.9762 & 6.0299 \\
\hline STD $-y$ & 5.9703 & 5.6863 \\
\hline
\end{tabular}

The results from targets 1 and 2 of the simulation without application level energy consideration and those from targets 1 thru 4 of energy aware simulation were selected 
for the summary in Table 5.6. It can be seen from the above table that the differences in the mean and the standard deviation of the tracking errors for the two scenarios were very small. The tracking performance in $x$ direction was slightly better in one scenario and the tracking performance in $y$ direction was slightly better in the other scenario. Thus, it can be concluded that there was no significant difference in tracking performance between the two scenarios.

\subsubsection{Evaluation of the Developed Energy Conservation Scheme}

The second part of the simulation was intended towards evaluating the energy conservation characteristics of the developed energy-efficient and distributed self-organization algorithm. The effect of the sleep-wakeup management scheme combined with the cross layer dynamic wakeup probability adjustment algorithm was studied in this section.

\subsubsection{Simulation Setup}

A sensor field of $500 \mathrm{~m}$ by $300 \mathrm{~m}$ was used and 4000 sensors were randomly deployed in the sensor field in a uniform manner for this simulation. The target path was the same as in Section 5.2.2. Similar to the previous simulation, multiple targets that followed the same path enter the sensor field one after another with a time separation of 50 seconds between each other.

The complete implementation of the DSOAAgent discussed in Section 5.1 was used for this simulation. The parameter values for the ns- 2 mobile node were the same as those used for simulation in Section 5.2.2. However, the complete default energy model was used for this simulation. Thus, the parameters shown in the following table were modified from the previous simulation. 
Table 5.7 Mobile Node Parameter Value Changes for Analyzing the Effect of the Cross Layer Power Management

\begin{tabular}{|c|l|c|}
\hline Parameter & \multicolumn{1}{|c|}{ Description } & Value \\
\hline EnergyModel & \multicolumn{2}{|c|}{$1.5 \mathrm{~J}$} \\
\hline initialEnergy & Initial energy level assigned to the node. & $20 \mathrm{~mW}$ \\
\hline idlePower & $\begin{array}{l}\text { Power consumption in idle state. The sleep } \\
\text { state in our simulations. }\end{array}$ & \\
\hline
\end{tabular}

In addition to the above settings, the nodes that were located in the boundary of the sensor network were assigned twice the energy level of the other nodes. This decision was taken because those nodes participate in both the intensive localization sensor group initialization process and the target tracking process. Thus, the sensor nodes deployed within 200 meters of the boundary of the sensor field (the $y$ axis of the sensor field in this simulation) were assigned $3.0 \mathrm{~J}$ of initial energy instead of $1.5 \mathrm{~J}$.

Three separate simulations were conducted in this section. In the first simulation, the wakeup probability of the sleep-wakeup management scheme was set to 1 . Thus, entire sensor network was kept awake all the time, and the sleep-wakeup management scheme was not utilized. In the second simulation, the sleep-wakeup management scheme was put into practice with a static wakeup probability. The wakeup probability of scheme was fixed to 0.55 , which was the lowest possible wakeup probability that enabled the successful target tracking. The third simulation employed the sleep-wakeup management scheme with dynamic wakeup probability adjustment. The proposed cross layer dynamic wakeup probability adjustment algorithm was used for dynamically updating the wakeup probability. The values used for the parameters of the message passing protocol were the same as those used in the previous simulation. For the cross layer wakeup probability adjustment algorithm, the parameter values listed in Table 5.8 were used. The number of 
targets that can be successfully tracked and the target tracking quality for each of the targets were recorded for all three simulations.

Table 5.8 Parameter Values of the Cross Layer Wakeup Probability Adjustment Algorithm

\begin{tabular}{|c|c|c|}
\hline Parameter & Description & Value \\
\hline \multicolumn{3}{|c|}{ Threshold Distances } \\
\hline$R_{\text {Low }}$ & See Figure 4.8 & $95 \mathrm{~m}$ \\
\hline$R_{\text {Med }}$ & See Figure 4.8. & $125 \mathrm{~m}$ \\
\hline$R_{H i g h}$ & See Figure 4.8 & $155 \mathrm{~m}$ \\
\hline \multicolumn{3}{|c|}{ Extreme Probability Limits } \\
\hline $\operatorname{Pr}_{\text {VeryLow }}$ & Lowest possible wakeup probability. & 0.005 \\
\hline $\operatorname{Pr}_{\text {VeryHigh }}$ & Highest assigned wakeup probability. & 0.8 \\
\hline \multicolumn{3}{|c|}{ Threshold Energy Limits } \\
\hline$E_{\text {VeryLow }}$ & Critically low threshold energy limit. & $0.35 \mathrm{~J}$ \\
\hline$E_{\text {Low }}$ & $\begin{array}{l}\text { First low energy limit that require applying higher } \\
\text { energy conservation patterns for the sensor node. }\end{array}$ & $0.5 \mathrm{~s}$ \\
\hline \multicolumn{3}{|c|}{ Weight Factors Used in Wakeup Probability Adjustment } \\
\hline$\lambda$ & $\begin{array}{l}\text { Energy saving factor for low energy highly } \\
\text { important nodes. }\end{array}$ & 0.5 \\
\hline$\gamma$ & $\begin{array}{l}\text { Energy saving factor for low energy medium } \\
\text { important nodes. }\end{array}$ & 0.2 \\
\hline$\delta$ & $\begin{array}{l}\text { Energy saving factor for low energy highly } \\
\text { important nodes. }\end{array}$ & 0.1 \\
\hline \multicolumn{3}{|c|}{ Network Connectivity Parameters } \\
\hline$N C_{\text {Comm }}$ & Parameter to define required network connectivity. & 24 \\
\hline$N C_{V H}$ & $\begin{array}{l}\text { Parameter to define wakeup probability for highly } \\
\text { important nodes. }\end{array}$ & 42 \\
\hline
\end{tabular}

The value of $R_{\text {Low }}$ was set $5 \%$ less than the value of $R_{F A R}$. The values for $R_{M e d}$ and $R_{H i g h}$ were set a communication range higher than the values of $R_{\text {Low }}$ and $R_{\text {Med }}$ respectively. The threshold energy limits and the weighting factors can be set using offline analysis, depending on the desired energy saving characteristics. The values for 
$N C_{C o m m}$ and $N C_{V H}$ too can be set using offline analysis, depending on desirable network connectivity. A good approximation for $N C_{V H}$ was 1.75 times the value of $N C_{C o m m}$.

\subsubsection{Results and Discussion}

The simulation results obtained from the three simulations are shown in the following figures. The target tracking performance in $x$ and $y$ directions for the three simulations are shown in Figures 5.12, 5.13, 5.15, 5.16, 5.18 and 5.19. In addition, typical energy footprints of the nodes in the sensor network after successfully tracking the last possible target for each of the three simulations are shown in Figures 5.14, 5.17 and 5.20.
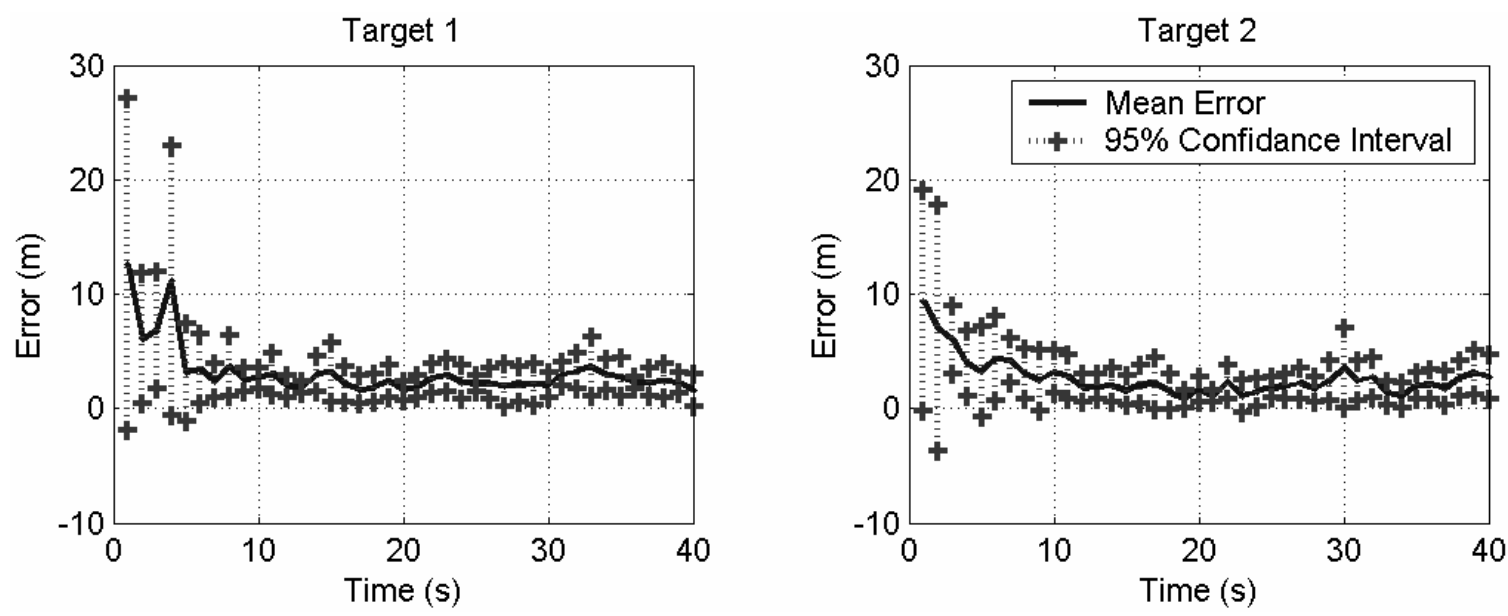

Figure 5.12 $x$ Direction Tracking Performance for No Sleep-wakeup Management Scenario
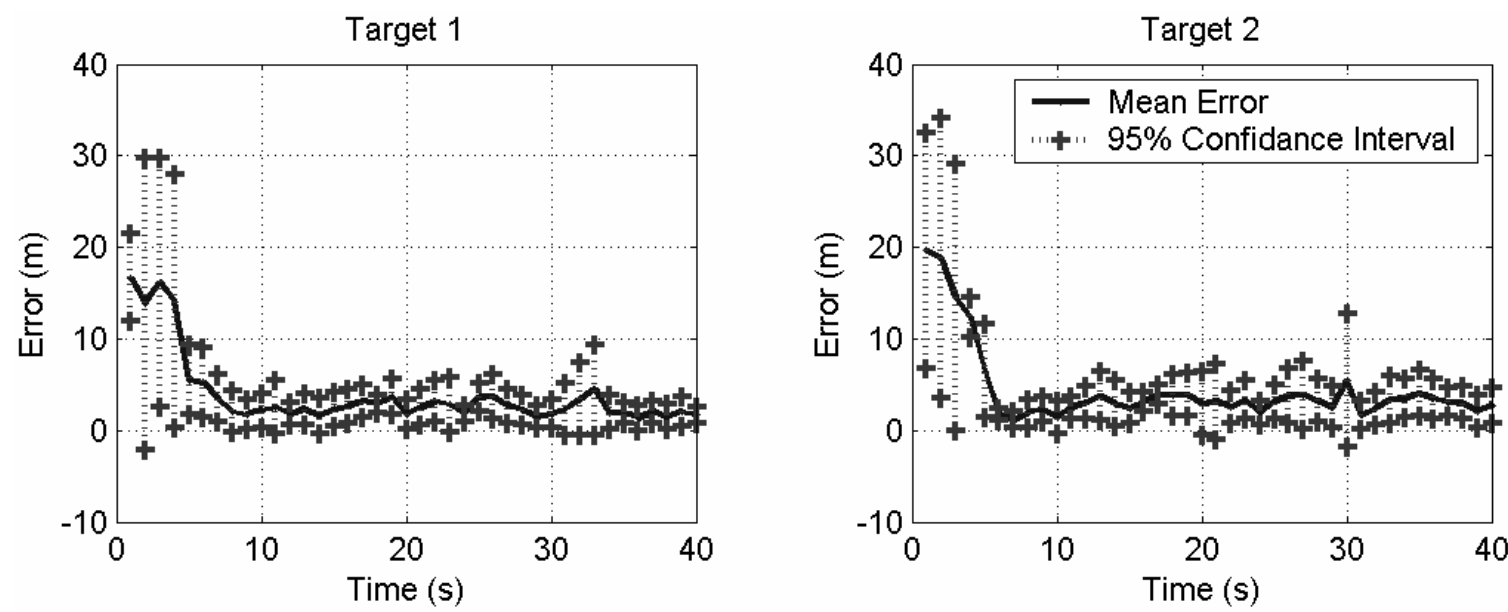

Figure 5.13 $y$ Direction Tracking Performance for No Sleep-wakeup Management Scenario 
When the sleep-wakeup management scheme was not utilized, the sensor network was able to track two targets successfully. Target tracking quality for the two targets in $x$ and $y$ directions are shown in Figures 5.12 and 5.13. Tracking more targets was not possible as most of the sensor nodes were out of battery power. This was evident from Figure 5.14, which illustrates a typical energy availability of the sensor nodes after tracking the second target.

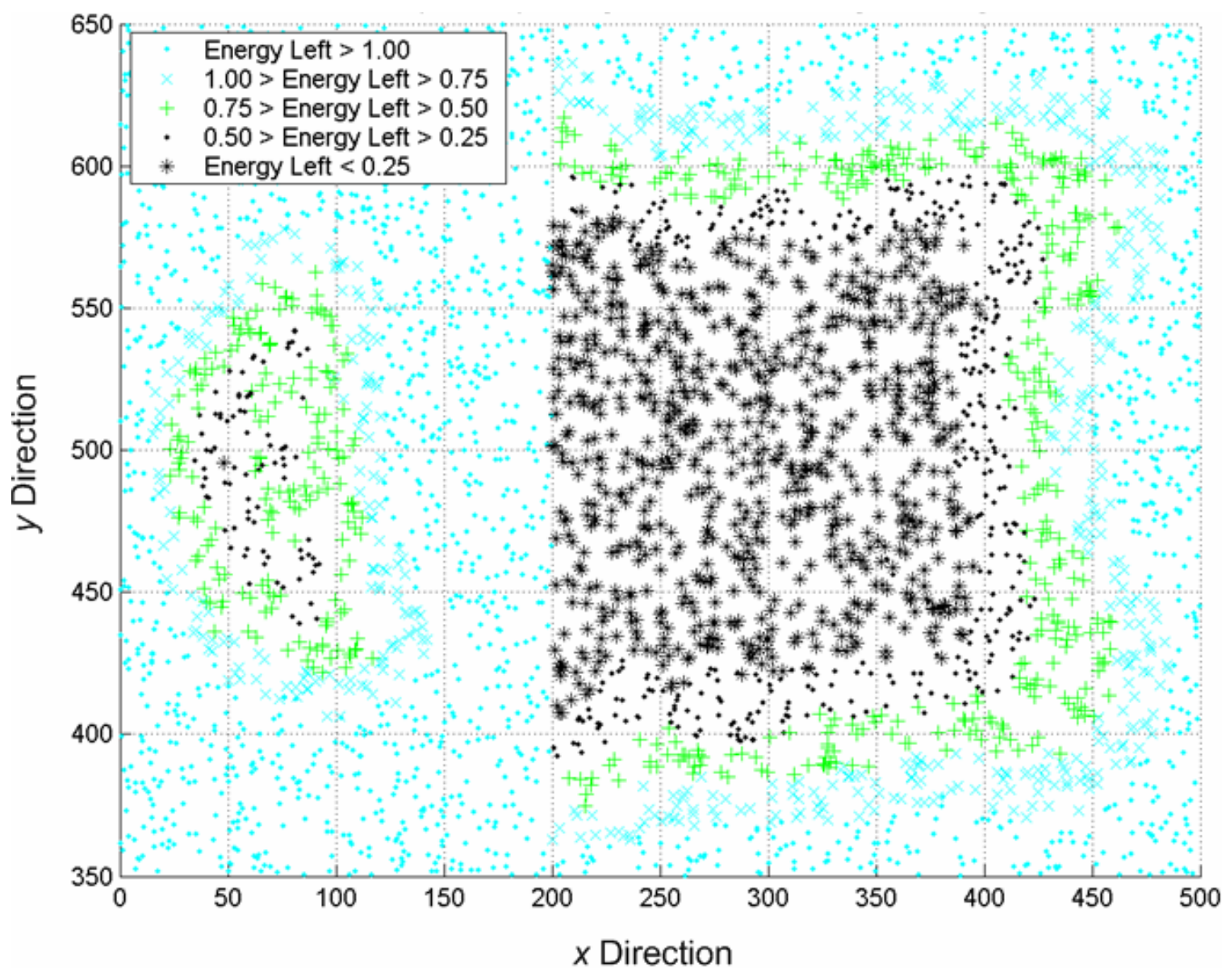

Figure 5.14 Sensor Node Energy Availability after Tracking the Second Target for No Sleep-wakeup Management Scenario (*Energy Level in Joules)

Figure 5.14 was an energy footprint of the network after tracking the second target, in a single run of the first simulation. It can be seen from this figure that most of the nodes in the sensor network, which were located either side of the target moving path 
and outside the boundary region of the network had less than $0.25 \mathrm{~J}$ of energy left in them. The nodes in this area were the ones which were participating in the target tracking and communicating activities. Although all the nodes did not participate in the target tracking and communicating activities, all of them lost energy as they were always awake. The nodes which were located near the boundary of the sensor field had more energy left as they were given double the amount of energy compared with the other nodes. However, some of these nodes too had only limited amount of energy left in them. The reason for this high energy usage was that those nodes had to participate in intensive localization sensor group initialization process as well as in the target tracking process.
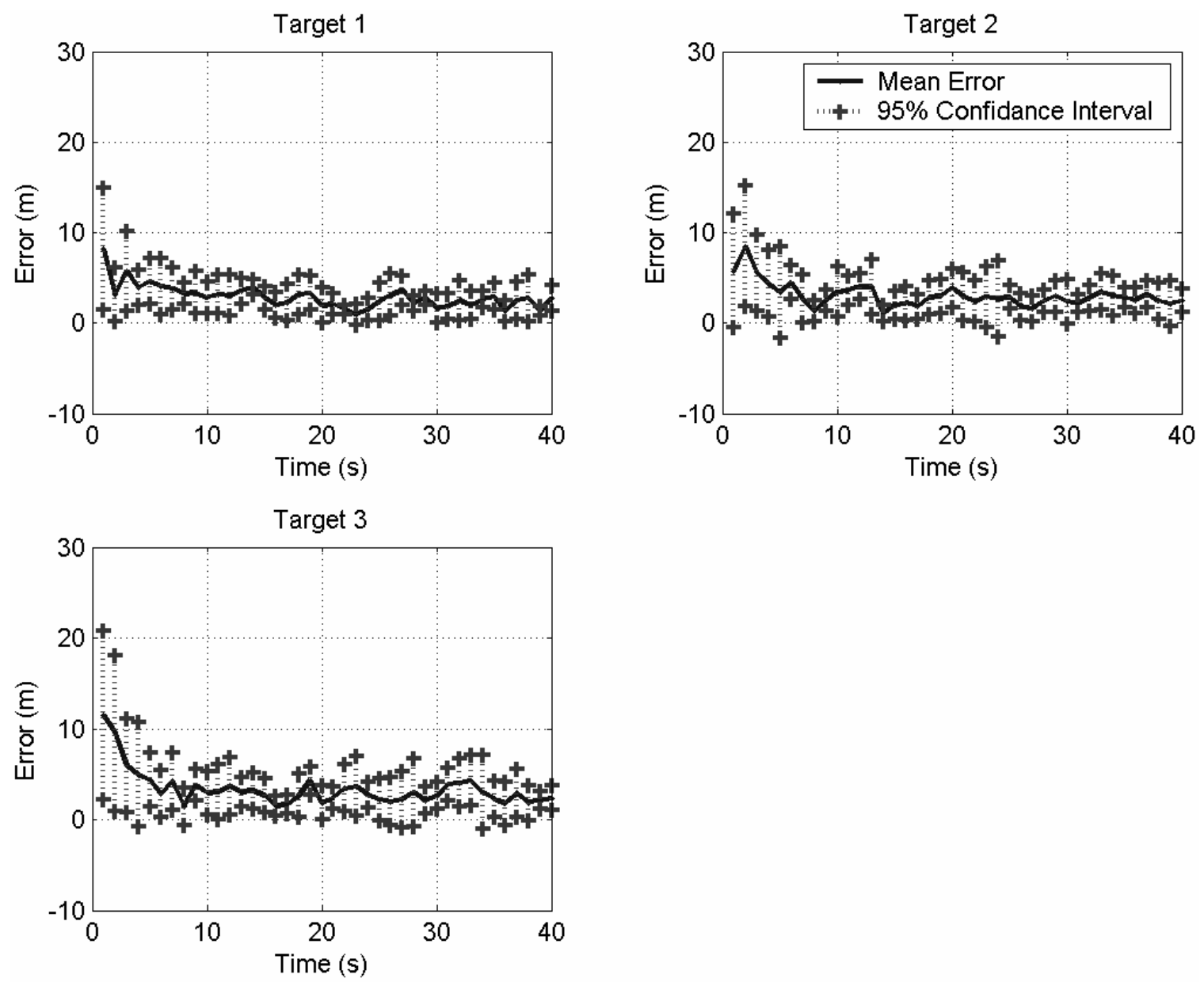

Figure 5.15 $x$ Direction Tracking Performance for the Fixed Wakeup Probability Scenario 

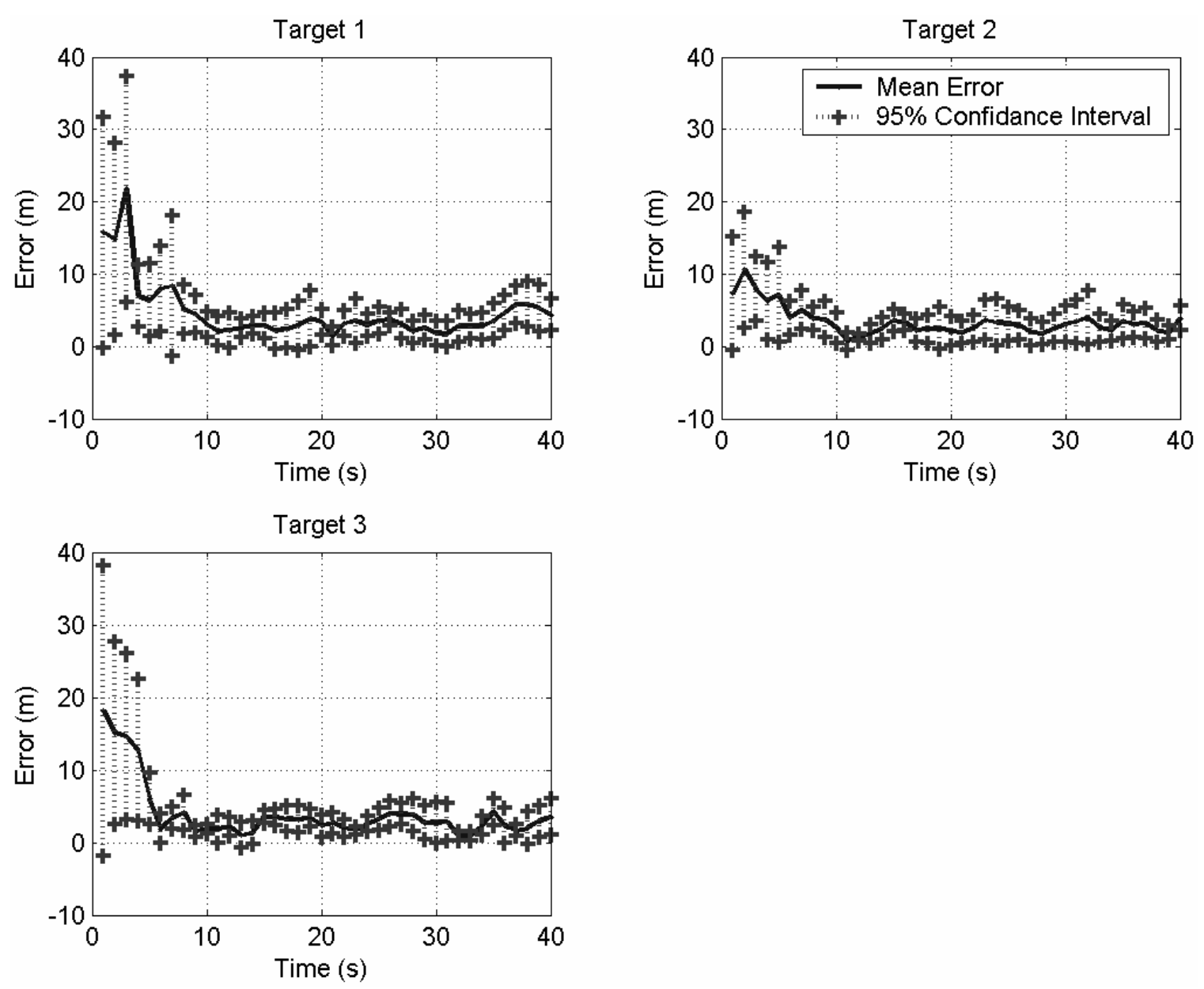

Figure 5.16 $y$ Direction Tracking Performance for the Fixed Wakeup Probability Scenario

Target tracking quality for the second simulation, where the sleep-wakeup management scheme was employed with a static wakeup probability of 0.55 , is shown in Figures 5.14 and 5.15. In this scenario, three targets were successfully tracked. Thus, it is evident that the incorporation of sleep-wakeup management scheme had resulted in extending the lifetime of the sensor network. Energy left in the sensor nodes after tracking the third target, for a single run is shown in the following figure. 


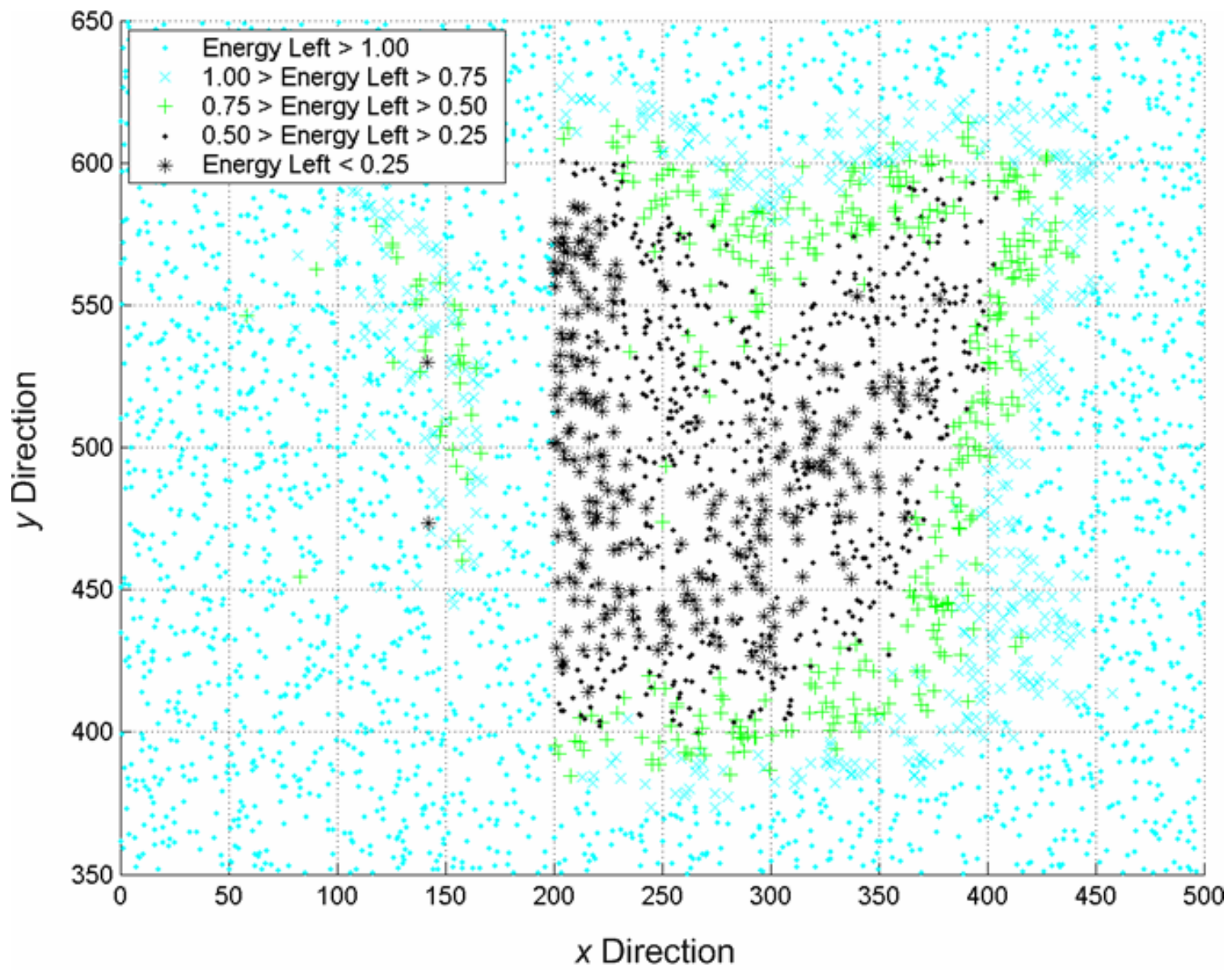

Figure 5.17 Sensor Node Energy Availability after Tracking the Third Target for the Fixed Wakeup Probability Scenario (*Energy Level in Joules)

It is evident from this figure that majority of the nodes, which were located alongside the path of the target in the middle of the sensor network had very low battery power. However, when compared to the no sleep-wakeup management scenario (Figure 5.14), it is evident that the condition of the sensor network was better in this scenario, even after tracking one more target. It can be clearly seen from the two figures that the higher number of nodes had more than $0.5 \mathrm{~J}$ of energy left in them (even after tracking one more target) when the sleep wakeup management scheme was incorporated with a fixed wakeup probability. This observation further supports the notion that the sleep-wakeup management scheme had resulted in extending the lifetime of the sensor 
network. It is evident from Figure 5.17 that few of the nodes which were close to the target path had more than $0.5 \mathrm{~J}$ of energy left. These energy savings were obtained due to the periodic sleeping of the sensor nodes. Although few of the nodes had more than $0.5 \mathrm{~J}$ of energy, the sensor network was not able to track more targets as the majority of the nodes, which were located around the target path, had low energy levels.
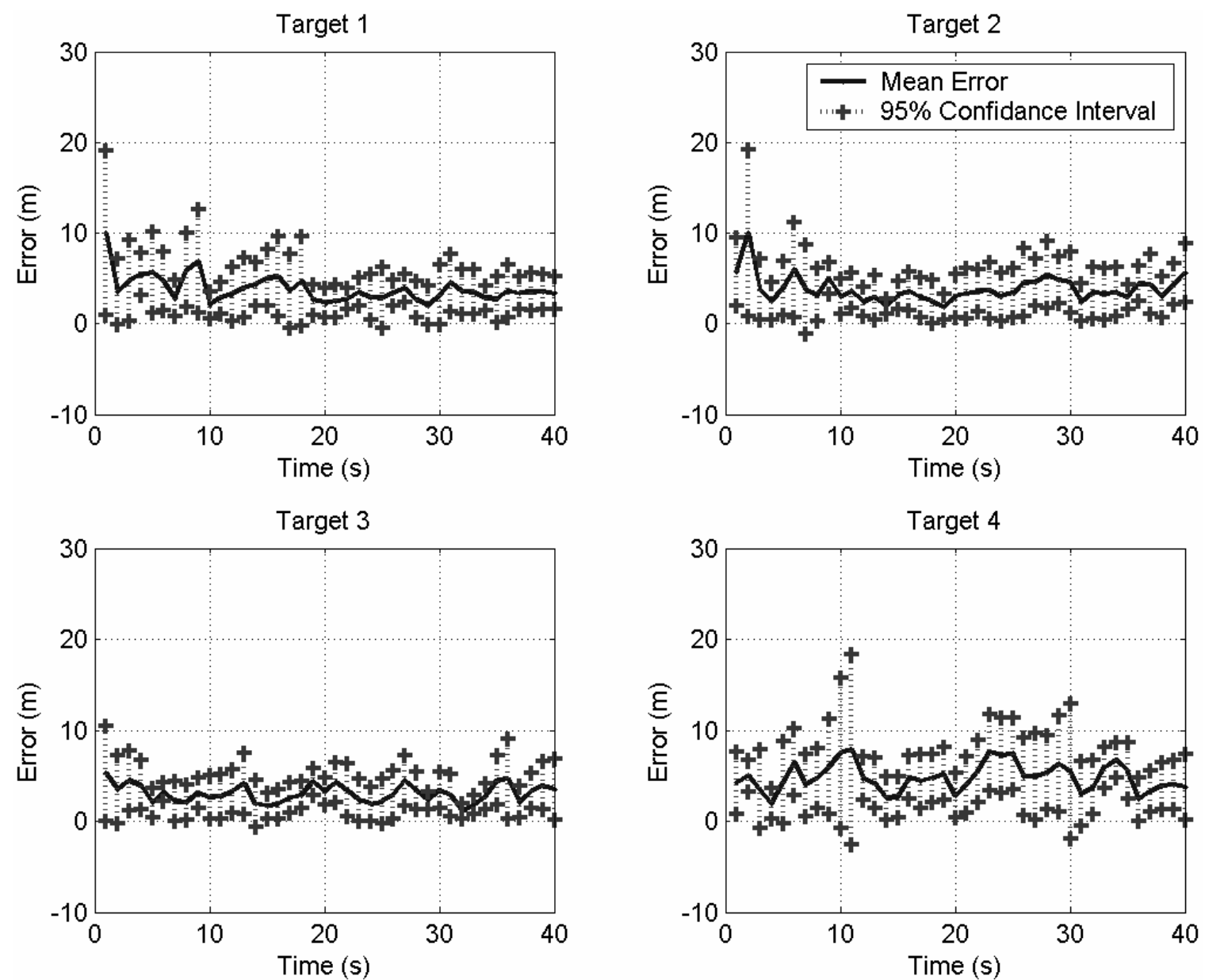

Figure 5.18 $x$ Direction Tracking Performance for the Dynamic Wakeup Probability Adjustment Scenario 

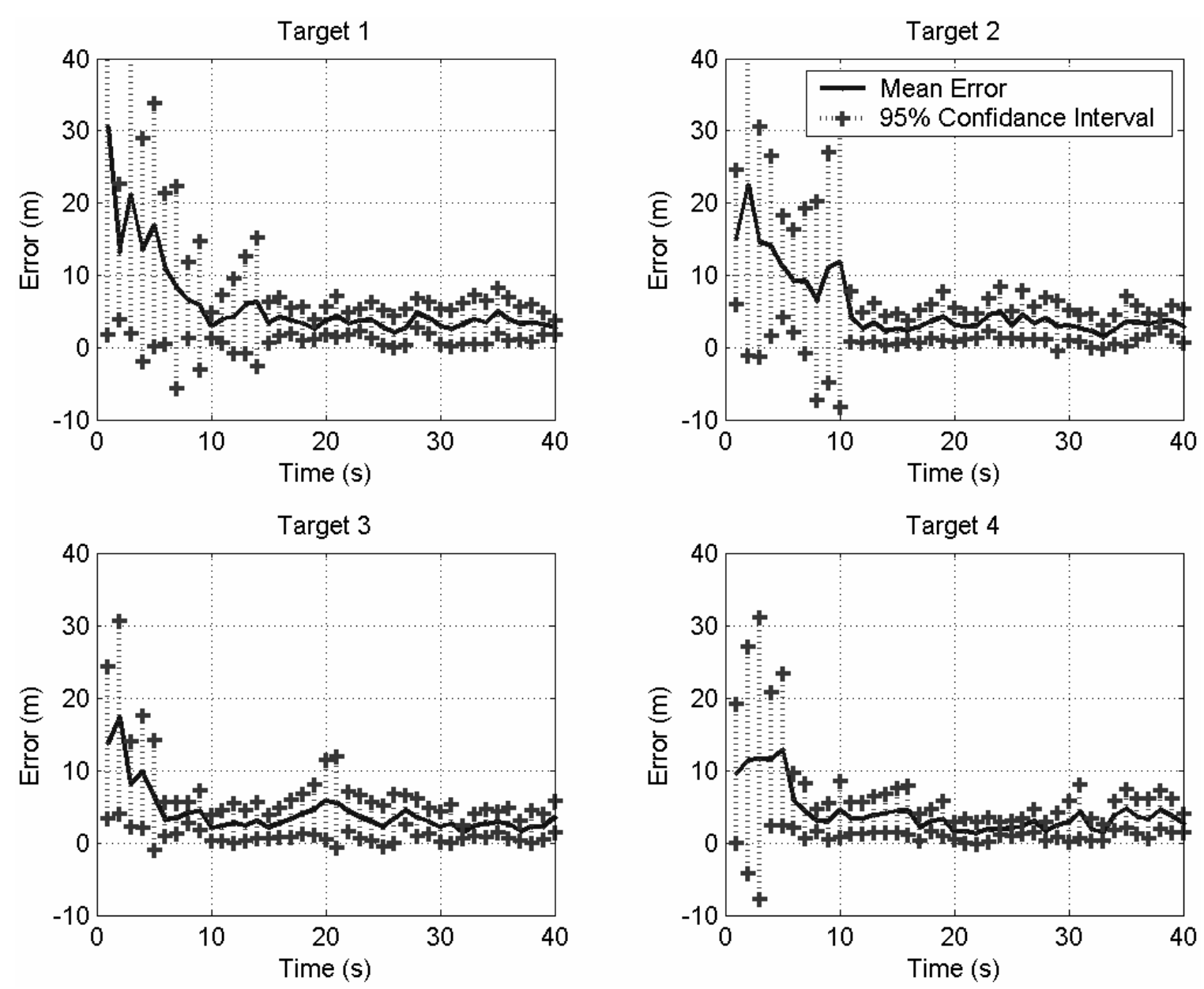

Figure 5.19 $y$ Direction Tracking Performance for the Dynamic Wakeup Probability Adjustment Scenario

When the proposed dynamic wakeup probability adjustment algorithm was utilized with the sleep-wakeup management scheme, four targets were tracked successfully. Target tracking performances for the four targets are summarized in Figures 5.18 and 5.19. Typical energy footprints of the nodes in the sensor network after successfully tracking the fourth target is shown in Figure 5.20. 


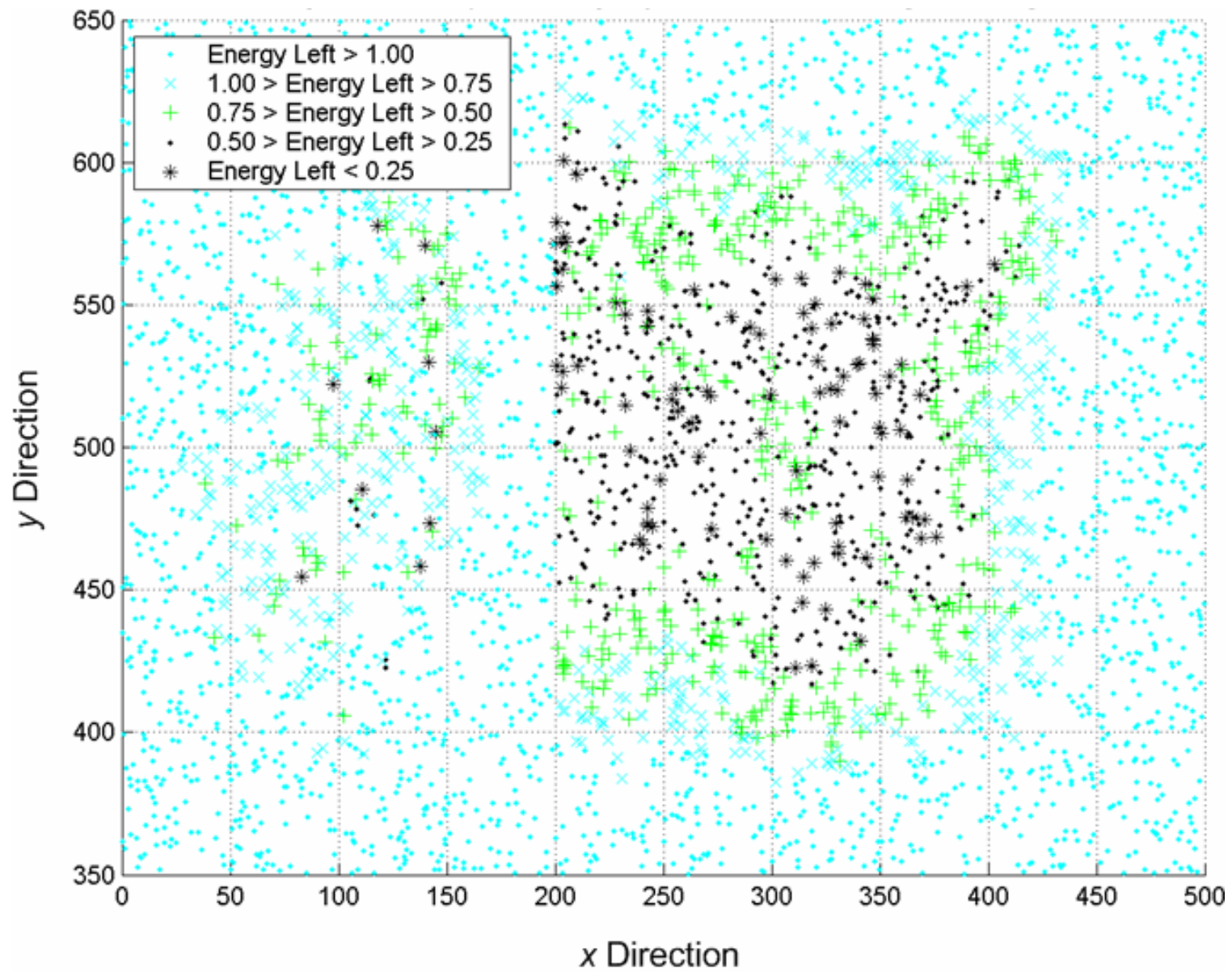

Figure 5.20 Sensor Node Energy Availability after Last Target Tracking for the Dynamic Wakeup Probability Adjustment Scenario (*Energy Level in Joules)

Compared with the energy availability of the two previous scenarios (shown in

Figures 5.14 and 5.17), the condition of the sensor network was superior, when the dynamic wakeup probability adjustment algorithm was utilized with the sleep-wakeup management scheme. The number of nodes, which were critical for tracking the target moving in this particular path and had more than $0.5 \mathrm{~J}$ of energy left in them, were higher than both the previous scenarios. This superior condition of the sensor network was after tracking four targets, which was one target more than the second scenario and two targets more than the first scenario. Thus, the dynamic updating of the wakeup probability had resulted in sensor nodes sleeping higher amount of time compared to the second scenario, 
where the sleep-wakeup scheme was utilized with a fixed wakeup probability. The dynamic wakeup probability adjustment algorithm was able to update the wakeup probability more closely to match the requirements of the tracking algorithm, which resulted in this improved energy savings. However, similar to the previous scenario, tracking of more targets was not possible due to lack sensor nodes with sufficient energy.

In addition, it can be seen from the above tracking results that degradation in target tracking performance due to the incorporation of sleep-wakeup management scheme was not significant. The average of the mean and the standard deviation of the target tracking error for the three scenarios are shown in Table 5.9.

Table 5.9 Summary of Tracking Performance Comparison for Three Power Management Scenarios (Average of the Mean and the Standard Deviation of Tracking Errors)

\begin{tabular}{|c|c|c|c|}
\hline Performance & No Sleep-wakeup & $\begin{array}{c}\text { Fixed Wakeup } \\
\text { Probability }\end{array}$ & $\begin{array}{c}\text { Dynamic Wakeup } \\
\text { Probability }\end{array}$ \\
\hline Mean- $x$ & 2.9011 & 3.1055 & 3.8834 \\
\hline Mean- $y$ & 4.1113 & 4.0406 & 4.9519 \\
\hline STD- $x$ & 2.1206 & 2.194 & 2.7434 \\
\hline STD- $y$ & 2.9397 & 2.8281 & 4.1153 \\
\hline
\end{tabular}

These results show that there was no significant degradation in target tracking quality when the sleep-wakeup management scheme was incorporated with a fixed wakeup probability. The target tracking quality had degraded slightly in $x$ direction while the target tracking quality in $y$ direction has shown a slight improvement. This indicates that the change in tracking quality was negligible. When the dynamic wakeup probability adjustment algorithm was utilized with the sleep-wakeup management scheme, there was a slight degradation in the target tracking quality. This degradation was mainly caused by the lower sensor node densities due to the nodes sleeping with a higher probability. 
However, the degradation was not significant and the target tracking quality was maintained at a satisfactory level. Therefore, the results of these simulations confirm the effectiveness of the proposed power management scheme in achieving extended network lifetime without a significant degradation of the target tracking performance.

When examining the target tracking results summarized in Figures 5.18 and 5.19, it can be seen that the large tracking errors were mainly present in the initial stage of the self-organization algorithm, where the four closest four closest nodes groups were employed for the target tracking. This was clearly evident in $y$ direction tracking performance. When the sensor node density was lower, the number of initial four closest nodes groups formed was lower. As these groups depend on the neighboring groups to improve the target tracking performance, fewer groups resulted in higher tracking errors. This was the reason for the higher initial errors. Once the localization sensor group started the tracking, it took some time to recover from the undesirable effects of these large initial errors. However, as the time progressed, the target tracking quality had improved to a satisfactory level.

\subsubsection{The Selection of Localization Sensor Group}

The node selection process was made energy aware using several assumptions (Section 4.2.3). This will result in selecting a suboptimal localization sensor group for target tracking. However, under the assumptions made, the selected localization sensor group should be near-optimal. In order to verify whether the simulation results agree with our analysis, the details of the localization sensor groups formed during the third scenario of the above simulations were recorded. The localization sensor groups selected by the algorithm at three different target positions are shown in the following figures. 


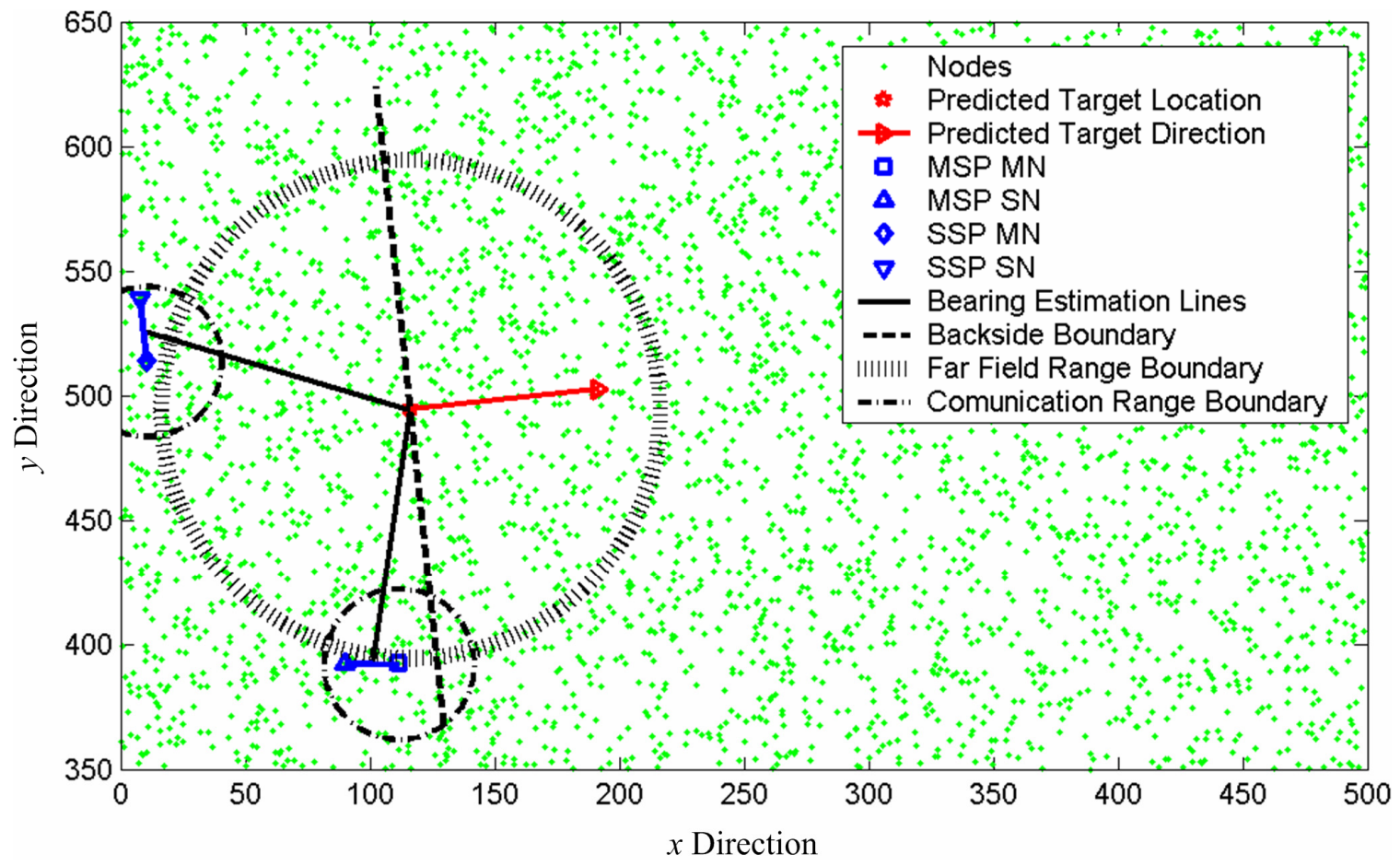

Figure 5.21 A Sample Localization Sensor Groups Selected at Time 11 (Group No 3)

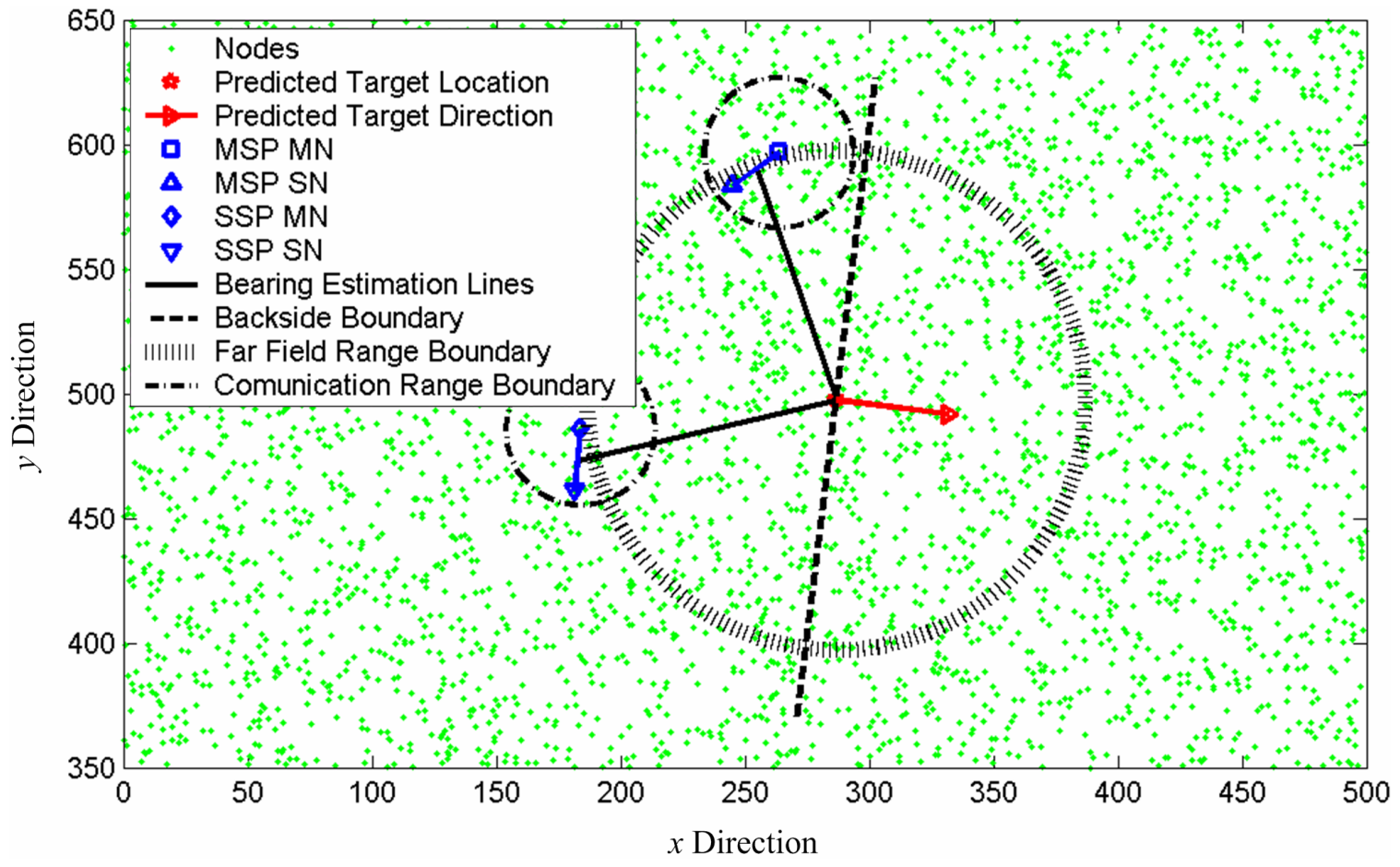

Figure 5.22 A Sample Localization Sensor Groups Selected at Time 29 (Group No 10) 


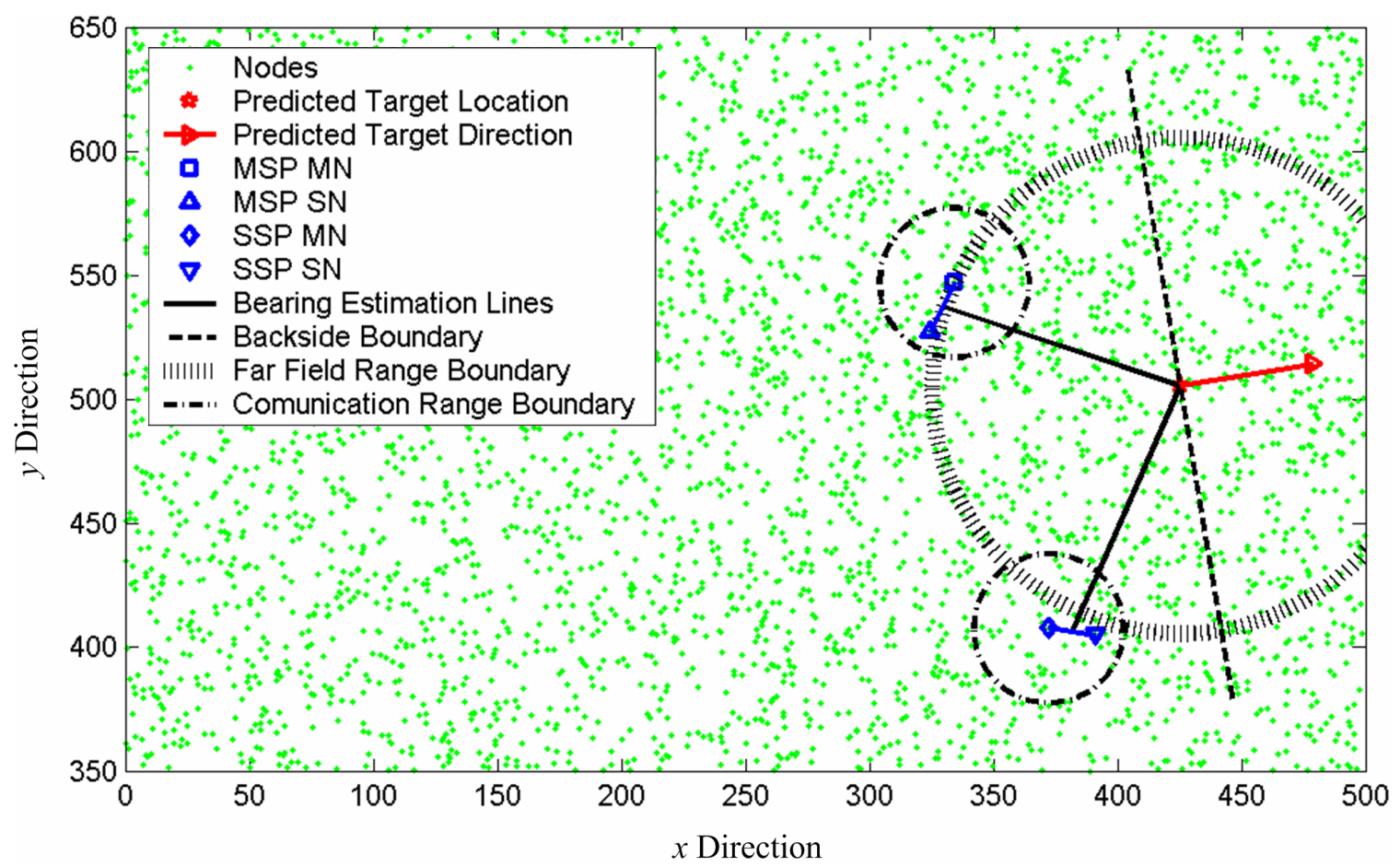

Figure 5.23 A Sample Localization Sensor Groups Selected at Time 42 (Group No 15)

It is evident from the above figures that the localization sensor groups were selected very close to the far field boundary. In addition the angles between the bearing lines of the selected localization sensor groups were close to $90^{\circ}$. Moreover, the selected sensor pairs were always located at the reverse side of the target moving direction. These observations were consistent with our analysis in Section 4.2. When considering the pairing of the sensor nodes, it can be seen that the slave sensor nodes were selected to maximize the distance between the two sensor nodes, while keeping the distance below the required threshold (indicated by the communication range boundary in the above figures). In addition, it is evident that the bearing angles of the sensor pairs were close to $90^{\circ}$. This confirms that the selection of slave nodes was performed to maximize the effective sensor pair baseline lengths, as expected in our analysis. Thus, as expected, the 
proposed energy efficient node selection criterion was capable of selecting the near-optimal localization sensor groups.

\subsubsection{Effect of Sensor Node Density}

Sensor node density is a very important factor that affects the target tracking performance of the developed self-organization algorithm. The localization sensor group selection process consists of searching for two master nodes and then the master nodes searching for their respective slave nodes. This energy aware node selection process was employed under the assumption that the sensor nodes were densely deployed, and results in selection of a sub-optimal localization sensor group. Thus, higher sensor node density is desirable for achieving near-optimal localization sensor groups. In addition, the higher sensor node density will increase the probability that the selected localization sensor group will be better suited for the target localization. The selection of a better localization sensor group will result in superior target tracking performance. In order to analyze the effect of sensor node on the target tracking performance, the following simulation was performed.

The simulation setup for this scenario was exactly similar to the simulation setup used in Section 5.2.3. However, in this simulation only one target was employed in contrast to tracking multiple numbers of targets. In addition, the number of sensors deployed in the sensor field was varied to achieve different sensor node densities. The target tracking performances of each run were recorded and the results obtained are shown in the following figures. 


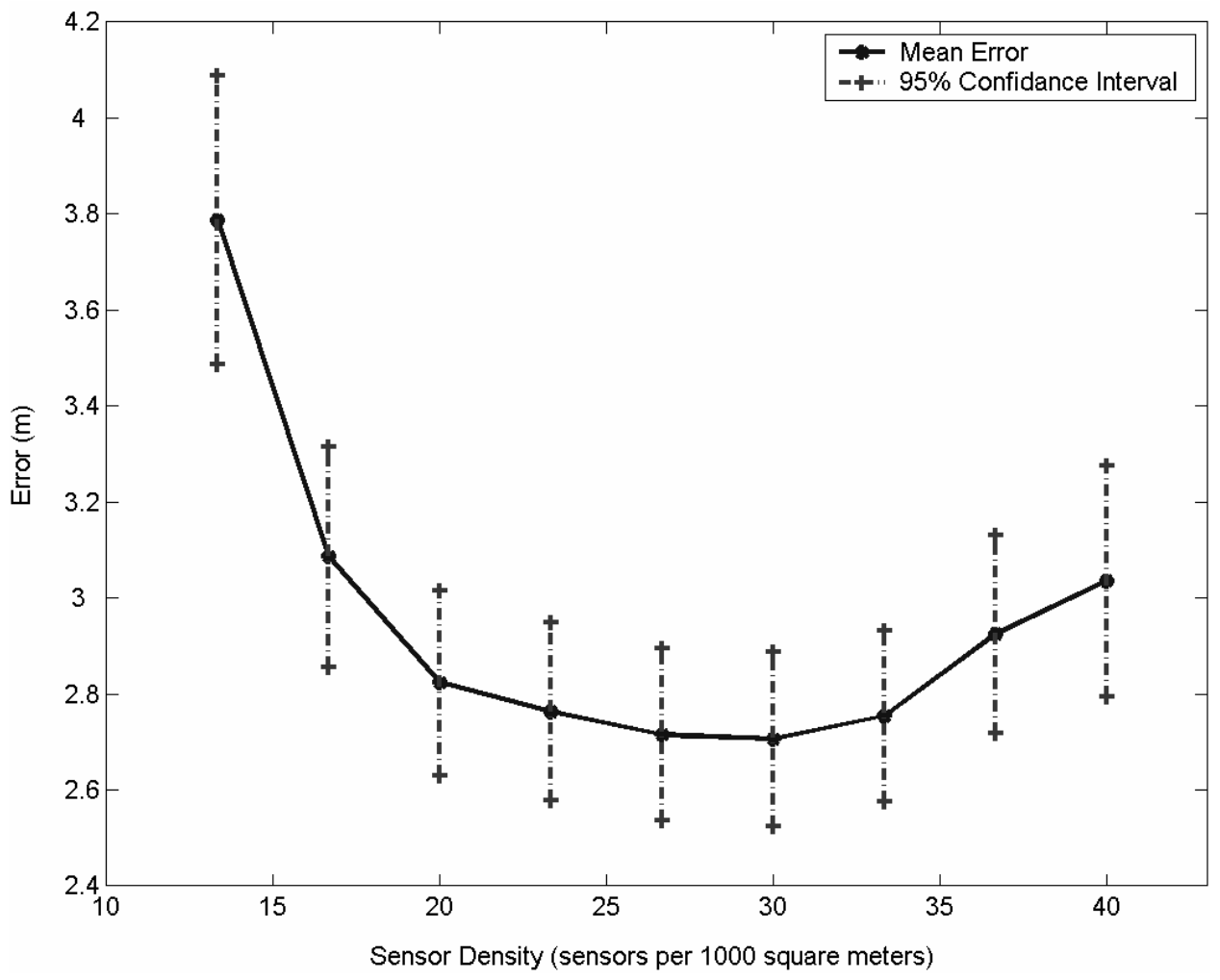

Figure 5.24 Effect of Sensor Node Density on $x$ Direction Target Tracking Performance

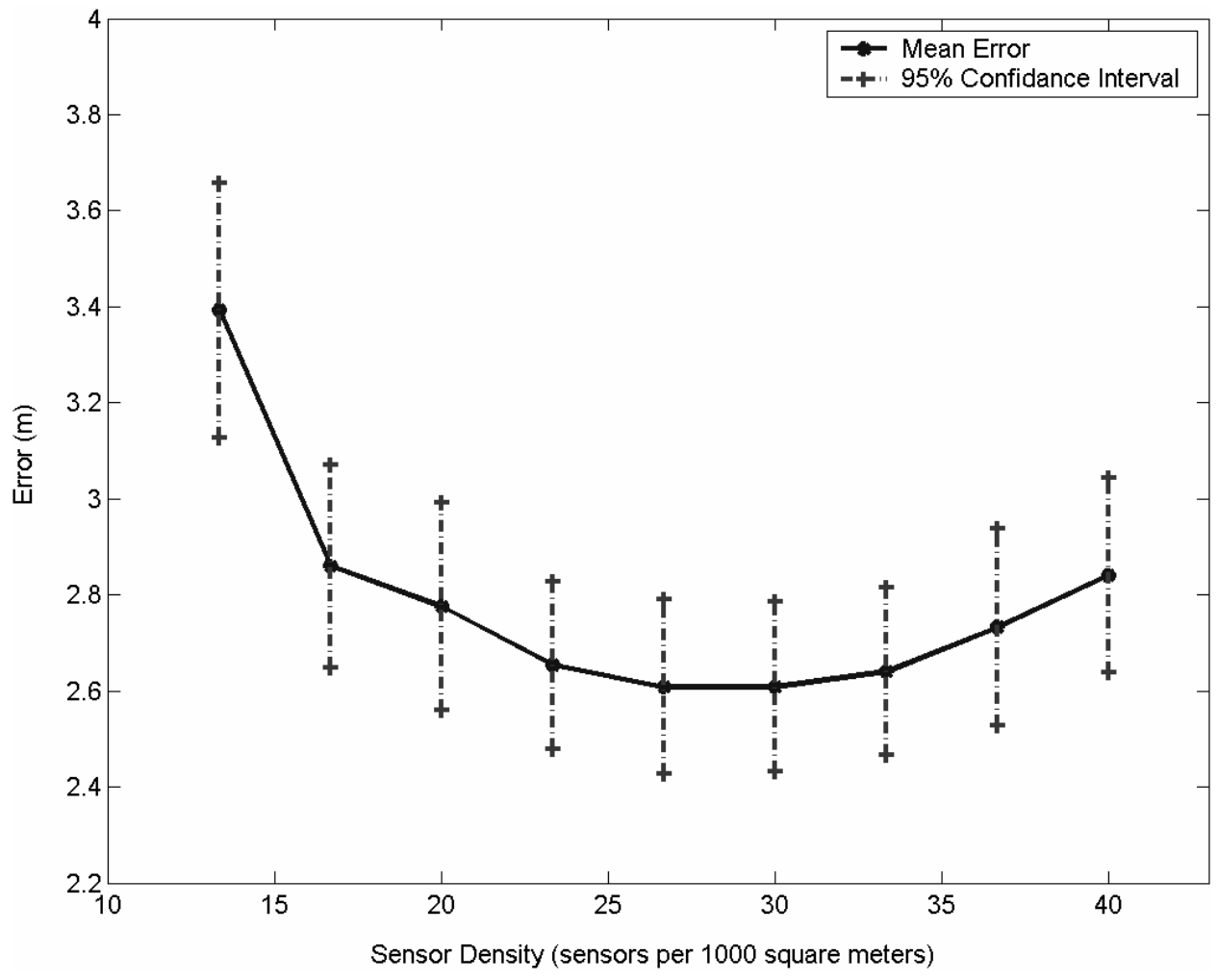

Figure 5.25 Effect of Sensor Node Density on $y$ Direction Target Tracking Performance 
It can be seen from the above results that initially, the increase in sensor node density resulted in lower localization errors. This was consistent with our previous analysis. Moreover, it was evident that there exists a threshold value of (20 sensors per 1000 square meters in this example) sensor node density, beyond which the increase in the sensor node density results in less significant improvement in target tracking performance. This was consistent with the phase transition phenomenon explained by Krishnamachari et al. [108]. This supports the notion that only a subset of nodes are required to be active in a densely deployed sensor network for the successful target tracking.

However, it can be identified that when the sensor node density was further increased, after some threshold value (30 sensors per 1000 square meters in this example) the tracking performance will start to degrade. This was resulted by the degradation in the communication quality as a result of increased sensor node density. When the sensor node density increases, the contention for communication between the nodes will increase. This would increase the collisions in the network and would result in increased communication delays.

The conclusions drawn from the above analysis further rationalize incorporation of sleep-wakeup management scheme to achieve energy conservation. 


\section{CHAPTER VI \\ CONCLUSIONS}

This research proposes an energy-efficient distributed self-organization algorithm for tracking ground targets using acoustic sensor networks with a single acoustic sensor at each sensor node. Bearing only measurements are used for the target localization. In contrast to traditional bearing only measurement based target tracking algorithms, which employ acoustic sensor arrays at each sensor node, our algorithm uses a single acoustic sensor at each sensor node. Our goal was to develop a self-organization algorithm which can dynamically organize the sensor nodes into virtual sensor arrays to perform the effective target tracking. In addition, the algorithm should be energy-efficient, scalable and should be able to operate under limited computing and communication resources. The proposed solution provides two major contributions, namely the self-organization algorithm and the cross-layer wakeup probability adjustment algorithm.

\subsection{Self-Organization Algorithm}

The major error sources of the localization process are studied, and nodes selection criterions that minimize the target localization errors are identified. A resource aware, distributed two-step node selection scheme is developed to select the sensor nodes for the localization sensor group. Using this node selection scheme, the self-organization algorithm selects a near-optimal localization sensor group to minimize the target tracking errors. A Kalman filter based tracking method is employed at the localization sensor group. The localization sensor group is dynamically updated to match the movements of the target to achieve better target tracking performance. A message passing protocol is developed to implement the self-organization algorithm in a distributed manner. The 
simulation results confirm that the target tracking algorithm provides satisfactory tracking performance. In addition, simulation results confirm the capability of the node selection scheme to select the near-optimal localization sensor group.

\subsection{Energy Conservation}

Energy efficiency is a major design constraint for sensor network applications and is pivotal for achieving extended sensor network lifetime. Thus, the energy-efficiency was given high priority in the design of the proposed self-organization algorithm. The energy considerations were incorporated into the node selection process to achieve a balanced energy usage throughout the sensor network, which will result in extended senor network life time. A sleep-wakeup management scheme was incorporated to the self-organization algorithm to reduce the power consumption. A novel cross layer wakeup probability adjustment algorithm was developed to achieve improved energy conservation. The algorithm dynamically adjusts the wakeup probability using the event information from the application layer and the network condition from the networking layers. The energy saving analysis confirms the effectiveness of the employed energy conservation mechanisms in extending lifetime of the sensor network with out degrading the target tracking quality.

\subsection{Future Work}

In this dissertation we have developed and evaluated an energy-efficient distributed self-organization algorithm for ground target tracking. Although the self-organization algorithm is complete, we believe there are several areas that deserve further considerations. 
- Limits of the Algorithm: In this research, we have not studied the target tracking limits of the algorithm. The considerations such as highest sampling rate the algorithm can operate, and the main factors that will affect the highest sampling rate the algorithm can operate, would be important issues for future research.

- Robustness of the Algorithm: Robustness of the Algorithm: Robustness is an important design parameter for sensor network applications. Thus, analyzing robustness of the algorithm and studying the techniques that could improve the robustness of the algorithm will be an interesting area of research.

- Multiple Target Tracking: The current version of the self-organization algorithm is capable of only tracking a single target. However, the ability to track multiple targets will be attractive for applications such as battlefield awareness. Thus, extending the algorithm to track multiple targets will be an important extension to our work. 


\section{REFERENCES}

[1] Akyildiz, I.F., Weilian, S., Sankarasubramaniam, Y. and Cayirci, E., "A Survey on Sensor Networks," IEEE Communication Magazine, vol. 40, pp. 102-114, Aug. 2002.

[2] Chong, C. and Kumar, S.P., "Sensor Networks: Evolution, Opportunities and Challenges," Proceedings of the IEEE, 91 (8), pp. 1247-1256, Aug. 2003.

[3] Hewish, M., "Reformatting fighter tactics," Janes International Defense Review, June 2001.

[4] Ash, J.N. and Moses, R.L., "Acoustic Time Delay Estimation and Sensor Network Self-Localization: Experimental Results," Journal of Acoustical Society of America, vol. 118 (2) , pp. 841-850, Aug. 2005.

[5] Allen, G.W., Lorincz, k., Welsh, M., Marcillo, O., Johnson, J., Ruiz, M. and Lees, J., "Deploying a Wireless Sensor Network on an Active Volcano," IEEE Internet Computing, 10 (2), pp. 18-25, March/April 2006.

[6] Brandstein, M.S., “A Framework for Speech Source Localization Using Sensor Arrays," Doctoral Dissertation, Brown University, May 1995.

[7] Kirkwood, B. C., "Acoustic Source Localization Using Time-Delay Estimation," Masters Thesis, Technical University of Denmark, Aug. 2003.

[8] Sheng, X and Hu Y.H., "Energy Based Acoustic Source Localization," Book Chapter in Information Processing in Sensor Networks, (Springer, Lecture Notes in Computer Science, IPSN 2003, LNCS 2634), vol. 2634, pp. 285-300, Jan. 2003.

[9] Kadar, I., "Optimum Geometry Selection for Sensor Fusion," Proceedings of the SPIE, vol. 3374, pp. 96-107, April 1998.

[10] Kaplan, L. M., "Global Node Selection for Localization in A Distributed Sensor Network," IEEE Transactions on Aerospace and Electronic Systems, vol. 42 (1), pp. 113-135, Jan. 2006.

[11] Kaplan, L. M., "Local Node Selection for Localization in A Distributed Sensor Network," IEEE Transactions on Aerospace and Electronic Systems, vol. 42 (1), pp. 136-146, Jan. 2006.

[12] Chu, M., Haussecker, H. and Zhao, F., "Scalable Information-Driven Sensor Querying and Routing for Ad Hoc Heterogeneous Sensor Networks," International Journal of High Performance Computing Applications, vol. 16, pp. 293-313, Aug. 2002. 
[13] Song, L. and Hatzinakos, D., "A Cross-Layer Architecture of Wireless Sensor Networks for Target Tracking," IEEE/ACM Transactions on Networking, vol. 15 (1), pp. 145-158, Feb. 2007.

[14] Gui, C. and Mohapatra, P., "Power Conservation and Quality of Surveillance in Target Tracking Sensor Networks," Proceedings of the 10th Annual International Conference on Mobile Computing and Networking, pp. 129-143, Sept. 2004.

[15] Pattem, S., Poduri, S. and Krishnamachari, B., "Energy-Quality Tradeoffs for Target Tracking in Wireless Sensor Networks," Book Chapter in Information Processing in Sensor Networks, (Springer, Lecture Notes in Computer Science, IPSN 2003, LNCS 2634), vol. 2634, pp. 32-46, Jan. 2003.

[16] Wan, Z., Zhang, J., Zhu, H., Makki, K. and Pissinou, N., "On Energy-Efficient and Low-Latency Medium Access Control in Wireless Sensor Networks," Proceedings of the 2008 IEEE Wireless Communications and Networking Conference (WCNC 2008), pp. 1905-1910, March/April 2008.

[17] Wan, Z., "Cooperative and Adaptive Medium Access Control in Multi-Hop Wireless Networks," Doctoral Dissertation, Florida International University, Nov. 2008.

[18] ns-2: User Information, Retrieved on September 20, 2009 from http://nsnam.isi.edu/nsnam/index.php/User_Information.

[19] Williams, S.M., "A Fully Distributed Method for Acoustic Localization with Sensor Networks," Masters Thesis, Vanderbilt University, Aug. 2005.

[20] Julian, P., Andreou, A.G., Riddle, L., Shamma, S., Goldberg, D.H. and Cauwenberghs, G., "A Comparative Study of Sound Localization Algorithms for Energy Aware Sensor Network Nodes," IEEE Transactions on Circuits and Systems I, vol. 51 (4), pp. 640-648, April 2004.

[21] Chen, J.C., Yao, K. and Hudson, R.E., "Source Localization and Beamforming," IEEE Signal Processing Magazine, vol. 19 (2), pp. 30-39, March 2002.

[22] Chen, J.C., Yao, K. and Hudson, R.E., "Maximum-Likelihood Source Localization and Unknown Sensor Location Estimation for Wideband Signals in the Near-Field," IEEE Transactions on Signal Processing, vol. 50 (8), pp. 30-39, Aug. 2002.

[23] Chen, J.C., Yao, K. and Hudson, R.E., "Acoustic Source Localization and Beamforming: Theory and Practice," EURASIP Journal on Applied Signal Processing, vol. 4, pp. 359-370, May 2003. 
[24] Chen, J.C., Yip, L., Elson, J., Wang, H., Maniezzo, D., Hudson, R.E., Yao, K. and Estrin, D., "Coherent Acoustic Array Processing and Localization on Wireless Sensor Networks," Proceedings of the IEEE, vol. 91 (8), pp. 1154-1162, Aug. 2003.

[25] Yao, K., Hudson, R.E., Reed, C.W., Chen, D. and Lorenzelli, F., "Blind Beamforming on a Randomly Distributed Sensor Array System," IEEE Journal on Selected Areas in Communications, vol. 16 (8), pp. 1555-1567, Oct. 1998.

[26] Brandstein, M.S., Adcock, J.E. and Silverman, H.F., "A Closed-Form Location Estimator for Use with Room Environment Microphone Arrays," IEEE Transactions on Speech and Audio Processing, vol. 5 (1) pp. 45-50, Jan. 1997.

[27] Ward, D.B. and Williamson, R.C., "Particle Filter Beamforming for Acoustic Source Localization in a Reverberant Environment," Proceedings of the IEEE International Conference on Acoustics, Speech, and Signal Processing, 2002 (ICASSP '02), vol. 2, pp. 1777-1780, May 2002.

[28] Biswas, P.K. and Phoha, S., "Self-Organizing Sensor Networks for Integrated Target Surveillance," IEEE Transactions on Computers, vol. 55 (8), pp. 10331047, Aug. 2006.

[29] Friedlander, D., Griffin, C., Jacobson, N., Phoha, S. and Brooks, R.R., "Dynamic Agent Classification and Tracking Using an Ad HocMobile Acoustic Sensor Network," EURASIP Journal on Applied Signal Processing, vol. 2003 (4), pp. 371-377, Jan. 2003.

[30] Ekman, M., Davstad, K. and Sjoberg, L., "Ground Target Tracking Using Acoustic Sensors," Proceedings of the Information, Decision and Control, 2007 (IDC '07), pp. 182-187, Feb. 2007.

[31] Ekman, M. and Bergman, N., "Ground Target Tracking with Acoustic Sensors using Particle Filters and Statistical Data Association," Proceedings of the 2006 IEEE Nonlinear Statistical Signal Processing Workshop, pp. 212-215, Sept. 2006.

[32] Wang, Q., Chen, W., Zheng, R., Lee, K. and Sha, L., “Acoustic Target Tracking Using Tiny Wireless Sensor Devices," Book Chapter in Information Processing in Sensor Networks, (Springer, Lecture Notes in Computer Science, IPSN 2003, LNCS 2634), vol. 2634, pp. 642-657, Jan. 2003.

[33] Sheng, $\mathrm{X}$ and Hu Y.H., "Dynamic Sensor Self-Organization for Distributive Moving Target Tracking," Journal of Signal Processing Systems, vol. 51 (2), pp. 161-171, May 2008. 
[34] Sheng, $\mathrm{X}$ and Hu Y.H., "Maximum Likelihood Multiple-Source Localization Using Acoustic Energy Measurements with Wireless Sensor Networks," IEEE Transactions on Signal Processing, vol. 53 (1), pp. 44-53, Jan. 2005.

[35] Chen, W.P., Hou, J.C. and Sha, L., "Dynamic Clustering for Acoustic Target Tracking in Wireless Sensor Networks," IEEE Transactions on Mobile Computing, vol. 3 (3), pp. 258-271, July 2004.

[36] Aurenhammer, F., "Voronoi Diagrams-A Survey of a Fundamental Geometric Data Structure," ACM Computing Surveys, vol. 23 (3), pp. 345-405, Sept. 1991.

[37] Kaplan, L.M. and Le, Q., "On Exploiting Propagation Delays for Passive Target Localization Using Bearings-Only Measurements," Journal of the Franklin Institute, vol. 342 (2), pp. 193-211, March 2005.

[38] Shi, L., Zhao, Z. and Tan, J., "Near Optimal Two-tier Target Tracking in Sensor Networks," Proceedings of the 2007 IEEE/RSJ International Conference on Intelligent Robots and Systems, (IROS 2007), pp. 1993-1996, Oct./Nov. 2007.

[39] Oh, S. and Sastry, S., "Tracking on a Graph," Proceedings of the 4th International Symposium on Information Processing in Sensor Networks, pp. 195-202, April 2005.

[40] Cevher, V., Sankaranarayanan, A.C., McClellan, J.H. and Chellappa, R., "Target Tracking Using a Joint Acoustic Video System," IEEE Transactions on Multimedia, vol. 9 (4), pp. 715-727, June 2007.

[41] Yu, Z., Dong, S. Wei, J., Xing, T. and Liu, H., "Neural Network Aided Unscented Kalman Filter for Maneuvering Target Tracking in Distributed Acoustic Sensor Networks," Proceedings of the International Conference on Computing: Theory and Applications (ICCTA'07), pp.245-249, March 2007.

[42] Li, D., Wong, K.D., Hu, Y.H. and Sayeed, A.M., "Detection Classification and Tracking of Targets in Distributed Sensor Networks," IEEE Signal Processing Magazine, vol. 19 (2), pp. 17-29, March 2002.

[43] Ramanathan, P., Saluja, K., Wang, K. and Clouqueur, T., "UW-API: A Network Routing Application Programmer's Interface," draft version 1.2, Oct. 2001. $\begin{array}{lllll}\text { (Retreived on } & \text { Sept. } & 2009 & \text { from }\end{array}$ http://www.ece.wisc.edu/ sensit/publications/uwapi1.2.pdf).

[44] Tseng, Y., Kuo, S., Lee, H. and Huang, C., "Location Tracking in a Wireless Sensor Network by Mobile Agents and Its Data Fusion Strategies," Springer, Lecture Notes in Computer Science : Information Processing in Sensor Networks (IPSN 2003), vol. 2634, pp. 625-641, April 2003. 
[45] Krishnamachari, B., Estrin, D. and Wicker, S., "The Impact of Data Aggregation in Wireless Sensor Networks," Proceedings of the 22nd International Conference on Distributed Computing Systems (ICDCSW '02), pp. 575-578, July 2002.

[46] He, T., Vicaire, P., Yan, T., Luo, L., Gu, L. Zhou, G., Stoleru, R., Cao, Q., Stankovic, J.A. and Abdelzaher, T., "Achieving Real-Time Target Tracking Using Wireless Sensor Networks," Proceedings of the 12th IEEE Real-Time and Embedded Technology and Applications Symposium (RTAS '06), pp. 37-48, April 2006.

[47] Gu, L., Jia, D., Vicaire, P., Yan, T., Luo, L., Tirumala, A., Cao, Q., He, T., Stankovic , J.A., Abdelzaher, T. and Krogh, B.H., "Lightweight Detection and Classification for Wireless Sensor Networks in Realistic Environments," Proceedings of the 3rd International Conference on Embedded Networked Sensor Systems (SenSys '05), pp. 205-217, Nov. 2005.

[48] He, T., Krishnamurthy, S., Stankovic, J.A., Abdelzaher, T., Luo, L., Stoleru, R., Yan, T., Gu, L., Hui, J. and Krogh , B., "Energy-Efficient Surveillance System Using Wireless Sensor Networks," Proceedings of the 2nd International Conference on Mobile systems, Applications and Services (MobiSys '04), pp. 270-283, June 2004.

[49] He, T., Krishnamurthy, S., Luo, L., Yan, T., Gu, L., Stoleru, R., Zhou, G., Cao, Q., Vicaire, P., Stankovic, J.A., Abdelzaher, T.F., Hui, J. and Krogh, B., "Vigilnet - An Integrated Sensor Network System for Energy-Efficient Surveillance," ACM Transactions on Sensor Networks, Vol. 2 (1), pp. 1-38, Feb. 2006.

[50] Ye, F., Zhong, G., Lu, S. and Zhang, L., “ PEAS: A Robust Energy Conserving Protocol for Long-Lived Sensor Networks," Proceedings of the 10th IEEE International Conference on Network Protocols, pp. 200-209, Nov. 2002.

[51] Xu, Y. and Lee, W., “ On Localized Prediction for Power Efficient Object Tracking in Sensor Networks," Proceedings of the 23rd International Conference on Distributed Computing Systems Workshops (ICDCSW'03), pp. 434-439, May 2003.

[52] Jiang, B., Han, K., Ravindran, B. and Cho, H., "Energy Efficient Sleep Scheduling Based on Moving Directions in Target Tracking Sensor Network," Proceedings of the 22nd IEEE International Symposium on Parallel and Distributed Processing (IPDPS 2008), pp. 1-10, April 2008.

[53] Jiang, B., Ravindran, B. and Cho, H., "Energy Efficient Sleep Scheduling in Sensor Networks for Multiple Target Tracking," Springer, Lecture Notes in Computer Science: Proceedings of the 4th IEEE International Conference on Distributed Computing in Sensor Systems, vol. 5067, pp. 498-509, June 2008. 
[54] Wang, X., Ma, J., Wang, S. and Bi, D., "Cluster-Based Dynamic Energy Management for Collaborative Target Tracking in Wireless Sensor Networks," Sensors, 7(7), pp. 1193-1215. July 2007.

[55] Heinzelman, W.R., Chandrakasan, A. and Balakrishnan, H. , "Energy-Efficient Communication Protocol forWireless Microsensor Networks," Proceedings of the 33rd Annual Hawaii International Conference on System Sciences, vol. 8, pp. 8020-8029, Jan. 2000.

[56] Yu, Z., Wei, J. and Liu H., "Energy-Efficient Collaborative Target Tracking Algorithm Using Cost-Reference Particle Filtering in Wireless Acoustic Sensor Networks," Journal of China Universities of Posts and Telecommunications, vol. 16 (1), pp. 9-15;43, Feb. 2009.

[57] Olule, E., Wang, G., Guo, M. and Dong, M., "RARE - An Energy-Efficient Target Tracking Protocol for Wireless Sensor Networks," Proceedings of the 2007 International Conference on Parallel Processing Workshops (ICPPW 2007), pp. 76-81, Sept. 2007.

[58] Cao, Q., Abdelzaher, T., He, T. and Stankovic, J., "Towards Optimal Sleep Scheduling in Sensor Networks for Rare-Event Detection," Proceedings of the 4th International Symposium on Information Processing in Sensor Networks (IPSN 2005), pp. 20-27, April 2005.

[59] Abrams, Z., Goel, A. and Plotkin, S., "Set K-Cover Algorithms for Energy Efficient Monitoring in Wireless Sensor Networks," Proceedings of the 3rd International Symposium on Information Processing in Sensor Networks (IPSN 2004), pp. 424-432, April 2004.

[60] Zhang, W. and Cao, G., "DCTC: Dynamic Convoy Tree-Based Collaboration for Target Tracking in Sensor Networks," IEEE Transactions on Wireless Communication, vol.3 (5), pp. 1689-1701, Sept. 2004.

[61] Xu, Y., Heidemann, J. and Estrin, D., "Geography Informed Energy Conservation for Ad Hoc Routing," Proceedings of the 7th ACM/IEEE Annual International Conference on Mobile Computing and Networking (ACM MobiCom), pp. 70-84, July 2001.

[62] Yang, L., Feng, C. Rozenblit, J.W. and Qiao, H., "Adaptive Tracking in Distributed Wireless Sensor Networks," Proceedings of the 13th Annual IEEE International Symposium and Workshop on Engineering of Computer Based Systems (ECBS 2006), pp 103-111, March 2006.

[63] Yeow, W., Tham, C. and Wong, W., "Energy Efficient Multiple Target Tracking in Wireless Sensor Networks," IEEE Transactions on Vehicular Technology, vol. 56 (2), pp. 918-928, March 2007. 
[64] Zhao, F., Shin, J. and Reich, J., "Information-Driven Dynamic Sensor Collaboration for Tracking Applications," IEEE Signal Processing Magazine, vol. 19 (2), pp. 61-72, March 2002.

[65] Liu, J., Reich, J. and Zhao, F., "Collaborative In-Network Processing for Target Tracking," EURASIP Journal on Applied Signal Processing, vol. 23 (4), pp. 378391, April 2003.

[66] Zhou, L. and Sen, A., "Adaptive Data Collectilon Scheme for Tracking Mobille Target in Wireless Sensor Networks," Proceedings of the 3rd Annual International Conference on Mobile and Ubiquitous Systems: Networking \& Services, pp. 1-10, July 2006.

[67] Oh, S., Sastry, S. and Schenato, L., "A Hierarchical Multiple-Target Tracking Algorithm for Sensor Networks," Proceedings of the 2005 IEEE International Conference on Robotics and Automation, pp. 2197-2202, April 2005.

[68] Oh, S., Russell, S. and Sastry, S., "Markov Chain Monte Carlo Data Association for General Multiple-Target Tracking Problems," Proceedings of the 43rd IEEE Conference on Decision and Control, pp. 735-742, Dec. 2004.

[69] Estrin, D., Govindan, R., Heidemann, J. and Kumar, S., "Next Century Challenges - Scalable Coordination in Sensor Networks," Proceedings of the ACM/IEEE International Conference on Mobile Computing and Networking, pp. 263-270, Aug. 1999.

[70] Lee, J., Cho, K., Lee, S., Kwon, T. and Choi, Y., "Distributed and EnergyEfficient Target Localization and Tracking in Wireless Sensor Networks," Computer Communications, vol. 29 (13-14), pp. 2494-2505, March 2006.

[71] Yu, X., Niyogi, K., Mehrotra, S. and Venkatasubramanian, N., "Adaptive Target Tracking in Sensor Networks," Proceedings of the 2004 Communication Networks and Distributed Systems Modeling and Simulation Conference (CNDS '04), Jan. 2004.

[72] Wagner, J. and Cristescu, R., "Power Control for Target Tracking in Sensor Networks," Proceedings of the 2005 Conference on Information Sciences and Systems (CISS 2005), March 2005.

[73] Cerpa, A. and Estrin, D., "ASCENT - Adaptive Self-Configuring sEnsor Networks Topologies," IEEE Transactions on Mobile Computing, vol. 3 (3), pp. 272-285, July/Aug. 2004. 
[74] Greunen, J.V., Petrović, D., Bonivento, A., Rabaey, J., Ramchandran, K. and Sangiovanni-Vincentelli, A., "Adaptive Sleep Discipline for Energy Conservation and Robustness in Dense Sensor Networks," Proceedings of the 2004 IEEE International Conference on Communications : Gate to the Global Information Society (ICC 2004), vol. 7, pp. 3657-3662, June 2004.

[75] Chen, B., Jamieson, K., Balakrishnan, H. and Morris, R., "SPAN-An EnergyEfficient Coordination Algorithm for Topology Maintenance in Ad Hoc Wireless Networks," ACM Wireless Networks, vol. 8 (5), pp. 481-494, Sept. 2002.

[76] Schurgers, C. Tsiatsis, V. Ganeriwal, S. and Srivastava, M., "Topology Management for Sensor Networks - Exploiting Latency and Density," Proceedings of the 3rd ACM International Symposium on Mobile Ad Hoc Networking \& Computing (MobiHoc '02), pp. 135-145, June 2002.

[77] Zheng, R., Hou, J.C. and Sha, L., "Asynchronous Wakeup for Ad Hoc Networks," Proceedings of the 4th ACM International Symposium on Mobile Ad Hoc Networking \& Computing (MobiHoc '03), pp. 35-45, June 2003.

[78] Deng, J., Han, Y.S., Heinzelman, W.D. and Varshneya, P.K., "Balanced-Energy Sleep Scheduling Scheme for High-Density Cluster-Based Sensor Networks," Computer Communications, vol. 28 (14), pp. 1631-1642, Sept. 2005.

[79] Xing, G., Lu, C., Zhang, Y., Huang, Q. and Pless, R., "Minimum Power Configuration in Wireless Sensor Networks," Proceedings of the 6th ACM International Symposium on Mobile Ad Hoc Networking and Computing, pp. 390-401, May 2005.

[80] Bandyopadhyay, S. and Coyle, E.J., "An Energy Efficient Hierarchical Clustering Algorithm for Wireless Sensor Networks," Proceedings of 22nd Annual Joint Conference of the IEEE Computer and Communications, vol. 3, pp. 1713-1723, March/April 2003.

[81] Ye, M. Li, C., Chen, G. and Wu, J., "EECS - An Energy Efficient Clustering Scheme in Wireless Sensor Networks," Proceedings of the 24th IEEE International Performance, Computing and Communications Conference (IPCCC 2005), pp. 535-540, April 2005.

[82] Heinzelman, W.B., Chandrakasan, A.P. and Balakrishnan, H., "An ApplicationSpecific Protocol Architecture for Wireless Microsensor Networks," IEEE Transactions on Wireless Communications, vol. 1 (4), pp. 660-670, Oct. 2002.

[83] Shah, R.C. and Rabaey, J.M., "Energy Aware Routing for Low Energy Ad Hoc Sensor Networks," Proceedings of the 2002 IEEE Wireless Communications and Networking Conference (WCNC2002), vol. 1, pp. 350-355, March 2002. 
[84] Schurgers, C. and Srivastava, M.B., "Energy Efficient Routing in Wireless Sensor Networks," Proceedings of the IEEE Military Communications Conference: Communications for Network-Centric Operations : Creating the Information Force (MILCOM 2001), vol. 1, pp. 357-361, Oct. 2001.

[85] Salhieh, A., Weinmann, J., Kochhal, M. and Schwiebert, L., "Power Efficient Topologies for Wireless Sensor Networks," Proceedings of the 2001 International Conference on Parallel Processing, pp. 156-166, Sept. 2001.

[86] Ye, W., Heidemann, J. and Estrin, D., "An Energy-Efficient MAC Protocol for Wireless Sensor Networks," Proceedings of the 21st Annual Joint Conference of the IEEE Computer and Communications Societies (INFOCOM 2002), vol. 3, pp. 1567-1576, June 2002.

[87] Ye, W., Heidemann, J. and Estrin, D., "Medium Access Control with Coordinated Adaptive Sleeping for Wireless Sensor Networks," IEEE/ACM Transactions on Networking, vol. 12 (3), pp. 493-506, June 2004.

[88] Lu, G., Krishnamachari, B. and Raghavendra, C.S., "An Adaptive EnergyEfficient and Low-Latency MAC for Data Gathering in Wireless Sensor Networks," Proceedings of the 18th International Parallel and Distributed Processing Symposium, pp. 224-231, April 2004.

[89] Dam, T.V. and Langendoen, K., "An Adaptive Energy-Efficient MAC Protocol for Wireless Sensor Networks," Proceedings of the 1st International Conference on Embedded Networked sensor Systems (SenSys '03), pp. 171-180, Nov. 2003.

[90] Polastre, J. Hill, J. and Culler, D., "Versatile Low Power Media Access for Wireless Sensor Networks," Proceedings of the 2nd International Conference on Embedded Networked Sensor Systems (SenSys '04), pp. 95-107, Nov. 2004.

[91] Liu, S., Fan, K. and Sinha, P., "CMAC - An Energy Efficient MAC Layer Protocol Using Convergent Packet Forwarding for Wireless Sensor Networks," Proceedings of the IEEE Communication Society Subject Matter Experts (SECON 2007), pp. 11-20, June 2007.

[92] Kannan, R. Kalidindi, R. Iyengar, S.S. and Kumar, V., "Energy and Rate based MAC Protocol for Wireless Sensor Networks," ACM SIGMOD: Special Section on Sensor Network Technology and Sensor Data Managment, vol 32 (4), pp. 6065, Dec. 2003.

[93] Zorzi, M. and Rao, R.R., "Geographic Random Forwarding (Geraf) for Ad Hoc and Sensor Networks - Energy and Latency Performance," IEEE Transactions on Mobile Computing, vol. 2 (4), pp. 349-365, Oct./Dec. 2003. 
[94] Carter, G.C., "Coherence and Time Delay Estimation," Proceedings of the IEEE, vol. 75 (2), pp. 236-255, Feb. 1987.

[95] Knapp, C. and Carter, G., "The Generalized Correlation Method for Estimation of Time Delay," IEEE Transactions on Acoustics, Speech and Signal Processing, vol. 24 (4), pp. 320-327, Aug. 1976.

[96] Carter, G., “ Time Delay Estimation for Passive Sonar Signal Processing,” IEEE Transactions on Acoustics, Speech and Signal Processing, vol. 29 (3), pp. 463470, June 1981.

[97] Weiss, A. and Weinstein, E., "Fundamental Limitations in Passive Time-Delay Estimation-Part I - Narrow-Band Systems," IEEE Transactions on Acoustics, Speech and Signal Processing, vol. 31 (2), pp. 472-486, April 1983.

[98] Quazi, A., "An Overview on the Time Delay Estimate in Active and Passive Systems for Target Localization," IEEE Transactions on Acoustics, Speech and Signal Processing, vol. 29 (3), pp. 527-533, June 1981.

[99] Kozick, R.J. and Sadler, B.M., "Source Localization with Distributed Sensor Arrays and Partial Spatial Coherence," IEEE Transactions on Signal Processing, vol. 52 (3), pp. 601-616, March 2004.

[100] Burne, R. A., Kadar, I., and Buczak, A., "A Self-Organizing, Cooperative Sensor Network for Remote Surveillance: Target Tracking While Optimizing the Geometry Between Bearing-Reporting Sensors and the Target," Proceedings of the SPIE, vol. 4393, pp. 173-182, April 2001.

[101] Torrieri, D.J., "Statistical Theory of Passive Location Systems," IEEE Transactions on Aerospace and Electronic Systems, vol. 20 (2), pp. 183-198, March 1984.

[102] Weiss, A.J., "Composite Bound on Arrival Time Estimation Errors," IEEE Transactions on Aerospace and Electronic Systems, vol. 22 (6), pp. 751-756, Nov. 1986.

[103] Spiesberger, J.L., "Locating Animals from Their Sounds and Tomography of the Atmosphere: Experimental Demonstration," Journal of Acoustical Society of America, vol. 106 (2), pp. 837-846, Aug. 1999.

[104] Smith, D. and Singh, S., "Approaches to Multisensor Data Fusion in Target Tracking - A Survey," IEEE Transactions on Knowledge and Data Engineering, vol. 18 (12), pp. 1696-1710, Dec. 2006.

[105] Chang, C. and Tabaczynski, J., "Application of State Estimation to Target Tracking," IEEE Transactions on Automatic Control, vol. 29 (2), pp. 98-109, Feb. 1984. 
[106] Akyildiz, I. F., Su, W., Sankarasubramaniam, Y. and Cayirci, E., "Wireless Sensor Networks - A Survey," Computer Networks, vol. 38 (4), pp. 393-422, March 2002.

[107] Anastasi, G., Conti, M., Francesco, M.D. and Passarella, A., "Energy Conservation in Wireless Sensor Networks: A survey," Ad Hoc Networks, vol. 7 (3), pp. 537-568, May 2009.

[108] Krishnamachari, B., Wicker, S.B. and Bejar, R., "Phase Transition Phenomena in Wireless Ad Hoc Networks," Proceedings of the 2001 IEEE Global Telecommunications Conference, vol. 5, pp. 2921-2925, Nov. 2001.

[109] Ramadurai, V. and Sichitiu, M.L., "Localization in Wireless Sensor Networks: A Probabilistic Approach," Proceedings of the 2003 International Conference on Wireless Networks (ICWN 2003), pp. 275-281, June 2003.

[110] Savvides, A., Han, C.C. and Srivastava, M.B., "Dynamic Fine-Grained Localization in Ad Hoc Wireless Sensor Networks," Proceedings of the 7th Annual ACM/IEEE International Conference on Mobile Computing and Networking (MobiCom 2001), pp. 166-179, July 2001.

[111] Moses, R.L., Krishnamurthy, D. and Patterson, R.M., "A Self-Localization Method for Wireless Sensor Networks," EURASIP Journal on Applied Signal Processing, vol. 4, pp. 348-358, March 2003.

[112] Savvides, A., Srivastava, M., Girod, L. and Estrin, D., "Localization in Sensor Networks," Book Chapter in Wireless Sensor Networks, pp. 327-349, Dec. 2006.

[113] Costa, J.A., Patwari, N. and Hero III, A.O., "Distributed WeightedMultidimensional Scaling for Node Localization in Sensor Networks," ACM Transactions on Sensor Networks, vol. 2 (1), pp. 39-64, Feb. 2006.

[114] Ganeriwal, S., Kumar, R. and Srivastava, M.B., "Timing-Sync Protocol for Sensor Networks," Proceedings of the ACM 1st International Conference on Embedded Networked Sensor Systems (SenSys 2003), pp. 138-149, Nov. 2003.

[115] Elson, J., Girod, L. and Estrin, D., "Fine-Grained Network Time Synchronization Using Reference Broadcasts," Proceedings of the 5th Symposium on Operating System Design and Implementation, vol. 36, pp. 147-163, Dec. 2002.

[116] Miklós Maróti, M., Kusy, B., Simon, G. and Lédeczi, A., "The Flooding Time Synchronization Protocol," Proceedings of the 2nd International Conference on Embedded Networked Sensor Systems, pp. 39-49, Nov. 2004.

[117] Sivrikaya, F. and Yener, B., "Time Synchronization in Sensor Networks: A Survey,” IEEE Network, vol. 18 (4), pp. 45-50, July/Aug. 2004. 
[118] Sichitiu, M.L. and Veerarittiphan, C., "Simple, Accurate Time Synchronization for Wireless Sensor Networks," Proceedings of the 2003 IEEE Wireless Communications and Networking Conference. vol. 2, pp. 1266-1273, May 2003.

[119] Sundararaman, B., Buy, U. and Kshemkalyani, A.D., "Clock Synchronization for Wireless Sensor Networks: A Survey,” Ad Hoc Networks, vol. 3 (3), pp. 281-323, May 2005.

[120] REAL 5.0 Overview, Retrieved on October 20, 2009 from http://www.cs.cornell.edu/skeshav/real/overview.html.

[121] CONSER Project Overview, Retrieved on October 20, 2009 from http://www.isi.edu/conser/overview.html.

[122] SAMAN Project Homepage, Retrieved on October 20, 2009 from http://www.isi.edu/saman/index.html.

[123] Monarch Project Homepage, Retrieved on October 20, 2009 from http://www.monarch.cs.rice.edu/.

[124] Fall, K. and Vardhan, K., "The NS Manual", The VINT Project - A Collaboration Between Researchers at UC Berkeley, LBL, USC/ISI and Xerox PARC, Jan. 2006. (Retreived on Oct. 20, 2009 from http://www.isi.edu/nsnam/ns/doc/ns_doc.pdf).

[125] Karp, B. and Kung, H.T., "GPSR: Greedy Perimeter Stateless Routing for Wireless Networks," Proceedings of the 6th Annual ACM/IEEE International Conference on Mobile Computing and Networking, pp. 243-254, Aug. 2000.

[126] Greedy Perimeter Stateless Routing (GPSR), Retrieved on October 20, 2009 from http://www.icir.org/bkarp/gpsr/gpsr.html.

[127] Walpola, M., Zhu, H. and Zhang, J., "Self Organization Algorithm for Unattended Acoustic Sensor Networks in Ground Target Tracking," Proceedings of the 2007 IEEE Wireless Communications and Networking Conference (WCNC 2007), pp. 2350-2354, March 2007.

[128] Zhang, J., Walpola, M., Roelant, D., Zhu, H. and Yen, K., "Self-Organization of Unattended Wireless Acoustic Sensor Networks for Ground Target Tracking," Journal of Pervasive and Mobile Computing, vol. 5 (2), pp. 148-164, April 2009. 
VITA

\section{MALAKA JAYATHU WALPOLA}

Nov. 2004

B.S., Computer Science and Engineering

University of Moratuwa

Katubedda, Sri Lanka

Dec. 2009

Doctoral Candidate, Electrical Engineering

Florida International University

Miami, FL

\section{PUBLICATIONS AND PRESENTATIONS}

Gamage, E.G.I.P., Lasantha, P.P.T., Sajeewa, B.G.S. and Walpola, M.J. "netSTAR Integrated Network Monitoring and Traffic Analysis System," Proceedings of the 6th International Information Technology Conference (IITC2004), Dec. 2004.

Walpola, M., Zhu, H. and Zhang, J., "Self Organization Algorithm for Unattended Acoustic Sensor Networks in Ground Target Tracking," Proceedings of the 2007 IEEE Wireless Communications and Networking Conference (WCNC 2007), pp. 2350-2354, March 2007.

Zhang, J., Walpola, M., Roelant, D., Zhu, H. and Yen, K., "Self-Organization of Unattended Wireless Acoustic Sensor Networks for Ground Target Tracking," Journal of Pervasive and Mobile Computing, vol. 5 (2), pp. 148-164, April 2009. 